\title{
VALUATION IDEALS IN POLYNOMIAL RINGS
}

\author{
BY \\ A. SEIDENBERG
}

Introduction. Among the valuation ideals in a polynomial ring $\mathfrak{D}=K[x, y]$ in two indeterminates, the ones of central importance are the simple valuation ideals, that is, the valuation ideals which are not products of two ideals different from $\mathfrak{D}$, since every valuation ideal has a unique factorization into simple valuation ideals.

The problem of the characterization of simple valuation ideals has been dealt with by Zariski, in the case that the field $K$ is algebraically closed and of characteristic 0 , in his paper, Polynomial ideals defined by infinitely near base points. There the problem is referred to the ring of holomorphic functions in $x, y: \mathfrak{D}^{*}=K\{x, y\}$, and a valuation ideal $\mathfrak{q}$ in $\mathfrak{D}^{*}$ is simple if and only if its general element is absolutely irreducible. If, however, only the characterization of the simple valuation ideals is desired, the notions of the general element of an ideal in $\mathfrak{D}^{*}$ and its absolute irreducibility are somewhat too strong for the problem set; although it should be stated that these notions are applied by Zariski to other topics not touched upon here.

In this paper we treat the theory of simple valuation ideals by a more explicit and direct method and we also extend the theory to algebraically closed fields of arbitrary characteristic $p \neq 0$. We characterize the simple $v$-ideals $\mathfrak{q}$ in the sequence of zero-dimensional valuation ideals in $\mathfrak{O}$, for a given valuation $v$, in terms of the value $v(\mathfrak{q}$ ) (under $v$ ) of $\mathfrak{q}$ (that is, the least value assumed by elements of $\mathfrak{q}$ ) and of the least value greater than $v(\mathfrak{q})$ assumed by elements of $\mathfrak{D}$. If $\mathfrak{q}$ is not simple, an explicit factorization for $q$ in terms of the two mentioned values is given

Since in our treatment the field $K$ is of arbitrary characteristic, Puiseux series expansions for valuations are not available. A corresponding tool is found in Theorem 6 . There for a given valuation $v$, a certain (finite or infinite) sequence of polynomials $f_{i}(x, y)$ is introduced. In the case that $K$ is of characteristic 0 , if $v$ is given $\left({ }^{1}\right)$, for example, by $y=c_{1} x^{r(1)}+c_{2} x^{r(2)}+\cdots, c_{i} \in K$, $r(i)=r_{i}$ rational, with $0<r_{i}<r_{i+1}$, then the polynomials $f_{i}(x, y)$ correspond roughly to the irreducible polynomials $g_{1}(x, y), g_{2}(x, y), \cdots$ which have $y=c_{1} x^{r(1)}, y=c_{1} x^{r(1)}+c_{2} x^{r(2)}, \cdots$ respectively as roots.

We next reduce our considerations to valuations of rational rank 2. This reduction serves two purposes. First it unifies the discussion; but much more

Presented to the Society, August 14, 1944; received by the editors September 30, 1944.

(1) For the representation of valuations by means of power series, in the case of ground field of characteristic 0 , see A. Ostrowski, Untersuchung zur arithmetische Theorie der Körper, part III, Math. Zeit. vol. 39 (1934). 
important is the following. If $v$ is of rational rank 2 then the value group contains irrational numbers, and if $\tau$ is the least irrational value assumed by elements of $\mathfrak{D}$, then the description of the valuation ideals in $\mathcal{O}$ for the valuation $v$ is intimately connected with the approximants and quasi approximants to a certain integral multiple of $\tau$.

Our treatment also provides a proof for the main theorem proved by Zariski in the case $p=0$, to the effect that under a quadratic transformation simple $v$-ideals are transformed into simple $v$-ideals.

1. Valuation-ideals and 0 -dimensional valuations. Let $\Sigma$ be a field containing a subfield $K$ and let $v$ be a valuation of $\Sigma$ over $K$, that is, a valuation in which the elements of $K$ other than zero have value zero. Let $\mathscr{B}$ be the valuation ring of $v$. Let $\mathfrak{O}$ be an integral domain contained in $\mathfrak{B}$. By the valuation ideals, or $v$-ideals, in $\bigcirc$ belonging to, or for, the valuation $v$ we mean the contracted ideals in $\mathfrak{O}$ of the ideals in $\mathfrak{B}$, that is, the ideal $\mathfrak{A}$ in $\mathfrak{O}$ is a $v$-ideal for the valuation $v$ if $\mathfrak{A}=\mathfrak{a} \cap \mathfrak{D}$, where $\mathfrak{a}$ is an ideal in $\mathfrak{B}$. It is clear that the $v$-ideals in $\mathfrak{O}$ belonging to $v$ may be characterized as the sets of elements in $\mathfrak{D}$ which contain together with any element all elements of $\mathcal{O}$ of equal or greater value. Another simple property of valuation ideals in $\mathfrak{D}$ dependent only on the fact that they are contracted ideals of ideals in an overlying valuation ring is the following. If $v^{\prime}$ is a second valuation of $\Sigma$ over $K$ with valuation ring $\mathfrak{B}^{\prime}$ such that $\mathfrak{O C} \mathfrak{B}^{\prime} \subset \mathfrak{B}$ then the valuation ideals in $\mathfrak{O}$ belonging to the valuation $v$ are among the valuation ideals in $\mathfrak{D}$ belonging to the valuation $v^{\prime}$. In fact, if $\mathfrak{A}=\mathfrak{a} \cap \mathfrak{D}, \mathfrak{a}$ an ideal in $\mathfrak{B}$, then also $\mathfrak{A}=\left(\mathfrak{a} \cap \mathfrak{B}^{\prime}\right) \cap \mathfrak{D}$, and $\mathfrak{a} \cap \mathfrak{B}^{\prime}$ is an ideal in $\mathfrak{B}^{\prime}$.

Let now $\mathfrak{O}$ be a finite integral domain over $K, \mathfrak{D}=K\left[x_{1}, \cdots, x_{k}\right]$, contained in $\mathfrak{B}$ (that is, $\mathfrak{D}$ consists of the polynomials in a finite number of elements of $\mathscr{B}$ with coefficients in $K$ ) and let $\Sigma$ be of finite degree of transcendency $n \geqq 1$ over $K$. By the dimension of $v$ is meant the degree of transcendency, over $K$, of the residue field of $v$. We assert that the valuation ideals in $\mathscr{D}$ belonging to $v$ are among the valuation ideals in $\mathfrak{O}$ belonging to a 0 -dimensional valuation $v^{\prime}$ of $\Sigma$ over $K$. In fact, if $v$ is not already 0 -dimensional, let $v_{0}$ be a 0 -dimensional valuation of $\mathfrak{B} / \mathfrak{B}$ over $K$, where $\mathfrak{B}$ is the ideal of non-units in $\mathfrak{B}$. It is well known $\left.{ }^{2}\right)$ that the two valuations $v$ and $v_{0}$ together determine a valuation $v^{\prime}$ of $\Sigma$ over $K$ such that $v^{\prime}(\alpha / \beta)=0$ if and only if $v(\alpha / \beta)=0$ and $v_{0}([\alpha / \beta])=0$; and such that $v^{\prime}(\alpha / \beta)>0$ if and only if $v(\alpha / \beta) \geqq 0$ and $v_{0}([\alpha / \beta])>0$, where $[\alpha / \beta]$ is the residue, or image, of $\alpha / \beta$ under the homomorphism $\mathfrak{B} \rightarrow \mathfrak{B} / \mathfrak{B}$. Clearly the valuation ring $\mathfrak{B}^{\prime}$ of $v^{\prime}$ is contained in $\mathscr{B}$ and $v^{\prime}$ is 0 -dimensional. Moreover $v_{0}$ can be taken such that $v^{\prime}\left(x_{i}\right) \geqq 0$, $i=1, \cdots, k$. In fact, since $v\left(x_{i}\right) \geqq 0$ it follows that the $v$-residues $\bar{x}_{i}$ of the $x_{i}$ are finite, and hence there exists a $v_{0}$ such that $v_{0}\left(\bar{x}_{i}\right) \geqq 0$, whence $v^{\prime}\left(x_{i}\right) \geqq 0$. $\mathfrak{D}$ is then contained in $\mathfrak{B}^{\prime}$, and this completes the proof of our assertion.

(2) This has reference to the process of composing $v$ and $v_{0}$. See Krull, Allgemeine Bewertunşstheorie, §5, Journal für Mathematik vol. 167 (1932). 
Below, $\Sigma$ is taken to be a pure transcendental extension of $K$ of degree 2, and $\subseteq$ to be a polynomial ring over $K$ in two indeterminates. As our purpose is a study of the $v$-ideals in $\mathfrak{O}$ belonging to valuations $v$ of $\Sigma$ over $K$, these valuations are restricted without loss of generality to be 0 -dimensional.

2. Valuation ideals in a ring with chain theorem. Let $v$ be a valuation of $\Sigma$ over $K$ with $v$-ring $\mathfrak{B}$, and let $\mathfrak{O}$ be an integral domain with chain theorem contained in $\mathfrak{B}$, and let the quotient field of $\mathfrak{O}$ be $\Sigma$. Then every $v$-ideal in $\mathfrak{O}$ belonging to $v$ has an immediate successor in the ordered set of v-ideals belonging to $v$. In fact, let $\left(\alpha_{1}, \cdots, \alpha_{k}\right)$ be a basis for an arbitrary ideal $\mathfrak{A}$ in $\mathfrak{D}$, and place $v(\mathfrak{U})=\min \left(v\left(\alpha_{1}\right), \cdots, v\left(\alpha_{k}\right)\right)$; it is immediate from the valuation axioms that $v(\mathfrak{A})$ is independent of the basis. If $v(\mathfrak{H})=v\left(\mathfrak{H}^{\prime}\right)$ for two ideals $\mathfrak{A}$ and $\mathfrak{A}^{\prime}$ in $\mathfrak{D}$, we also write $\mathfrak{A} \sim \mathfrak{A}^{\prime}$. Since $v$ is non-trivial (by assumption), there exists an element $\alpha$ of $\Sigma$ with $v(\alpha)>0$. Let $\alpha=f / g, f, g \in \mathcal{D}$; then $v(f)=v(\alpha)+v(g)>0$, since $g \in \mathfrak{D} \subset \mathfrak{B}$ implies $v(g) \geqq 0$. Thus there exist elements of value greater than $v(\mathfrak{A})$, for example, the elements of $\mathfrak{A} f$. Let $\mathfrak{A}$ be a $v$-ideal for $v$. The set of elements $\mathfrak{X}^{\prime}$ in $\mathfrak{D}$ of value greater than $v(\mathfrak{U})$ has the property that together with any element it contains all the elements of $\mathfrak{O}$ of equal or greater value. Hence $\mathfrak{A}^{\prime}$ is a $v$-ideal for $v$, and clearly $\mathfrak{A}^{\prime}$ is the immediate successor of $\mathfrak{A}$.

Let $v$ be 0 -dimensional, and consider the well-ordered set of $v$-ideals in $D$ belonging to $v$. The first $v$-ideal $q_{0}$ in $\mathfrak{D}$ is $\mathfrak{D}$ itself. The immediate successor $q_{1}$ of $q_{0}$ is the contraction to $\mathfrak{D}$ of the ideal of non-units $\mathfrak{B}$ in $\mathscr{B}$, and is a prime ideal in $\mathfrak{D}$. Moreover, it is 0-dimensional. In fact, since $\mathfrak{B} \cap \mathfrak{D}=\mathfrak{q}_{1}$, $\mathfrak{O} / \mathfrak{q}_{1} \subseteq \mathfrak{B} / \mathfrak{B}$, whence $\mathfrak{D} / \mathfrak{q}_{1}$ is algebraic over $K$, or in other words, $\mathfrak{q}_{1}$ is 0 -dimensional. The well-ordered set of $v$-ideals in $\mathfrak{D}$ belonging to $v$ starts with a simple sequence: $\mathfrak{q}_{0}, \mathfrak{q}_{1}, \mathfrak{q}_{2}, \ldots$ Each $\mathfrak{q}_{i}, i \geqq 1$, is a primary ideal with $\mathfrak{q}_{1}$ as associated prime ideal. In fact, $v\left(\mathfrak{q}_{1}\right)<v\left(\mathfrak{q}_{1}^{2}\right)<\cdots<v\left(\mathfrak{q}_{1}^{i}\right)$, whence $v\left(\mathfrak{q}_{1}^{i}\right) \geqq v\left(\mathfrak{q}_{i}\right)$; therefore $\mathfrak{q}_{1}^{i} \subseteq \mathfrak{q}_{i} \subseteq \mathfrak{q}_{1}$, whence $\mathfrak{q}_{i}$ is a primary ideal with $q_{1}$ as associated prime ideal.

The intersection $\Delta \mathfrak{q}_{i}$ of all the ideals $\mathfrak{q}_{i}$ is a prime ideal. In fact, if $a b \equiv 0\left(\Delta q_{i}\right), a \neq 0\left(\Delta q_{i}\right), b \not \equiv 0\left(\Delta q_{i}\right)$, then let $q_{r}$ be the last ideal in the sequence $\left\{q_{i}\right\}$ containing $a$, and $q_{s}$ the last ideal in the sequence $\left\{q_{i}\right\}$ containing $b$. Then $v(a)=v\left(\mathfrak{q}_{r}\right), v(b)=v\left(\mathfrak{q}_{s}\right)$, whence $v(a b)=v\left(\mathfrak{q}_{r} \mathfrak{q}_{s}\right)$. Since every $v$-ideal for $v$ contains together with any element all elements of equal or greater value, we have $q_{r} q_{s} \equiv 0\left(\Delta q_{i}\right)$. Since some power of $q_{1}$ is in $q_{r}$ and similarly for $q_{s}$, also some power of $q_{1}$ is in $\Delta q_{i}$, say $q_{1}^{n} \equiv 0\left(\Delta q_{i}\right)$. This is a contradiction, for if $q_{1}^{n} \equiv 0\left(q_{i}\right)$ then $i$ cannot exceed the length of $q_{1}^{n}$.

If $K$ is algebraically closed (and $v$ is 0 -dimensional) the sequence $q_{0}, q_{1}, \ldots$ is called a Jordan sequence, in view of the fact that in this case each $q_{i+1}$ is a maximal subideal of $\left(^{3}\right) q_{i}$.

We are particularly concerned below with all the above results in the case that $K$ is algebraically closed, of arbitrary characteristic, that $\Sigma$ is a pure

(3) O. Zariski, Polynomial ideals defined by infinitely near base points, Theorem 1, Amer. J. Math. vol. 60 (1938). 
transcendental extension of degree 2 , and that $\mathscr{D}$ is a polynomial ring in two indeterminates (generating elements of $\Sigma$ over $K$ ). These assumptions on $K$ and $\Sigma$ are assumed hereafter. Also any considered valuation $v$ of $\Sigma$ over $K$ is understood to be $\mathbf{0}$-dimensional.

In general, $\Delta \mathfrak{q}_{i}$ is prime. In the case that $\mathfrak{D}$ is a polynomial ring in two indeterminates, $\Delta \mathfrak{q}_{i}$ is either the ideal (0), or it is 1-dimensional, whence principal, say $\Delta q_{i}=(f)$. In the former case every $v$-ideal belonging to $v$ is in the sequence $\left\{q_{i}\right\}$. In the latter case every $v$-ideal belonging to $v$ is of the form $(f)^{n} \mathfrak{q}_{m}, n=0,1, \ldots ; m=0,1, \cdots$. As a consequence, the study of $v$-ideals in $\mathfrak{O}$ reduces essentially to the study of the 0 -dimensional $v$-ideals.

3. $v$-ideals and quadratic transformations. Let $v$ be a 0 -dimensional valuation of $\Sigma$ over $K$. Since any element or its reciprocal has non-negative $v$-value, $\Sigma$ has two generating elements $x, y$ with non-negative value. Let $\mathfrak{D}=K[x, y]$. The center of the valuation $v$ in $\mathfrak{D}$, that is, the contraction to $\mathscr{D}$ of the ideal of non-units in the $v$-ring $\mathfrak{B}$ of $v$, is the prime ideal $q_{1}$. Since $q_{1}$ is 0 -dimensional and $K$ is algebraically closed there exist constants $c, d$ such that $x \equiv c\left(q_{1}\right)$, $y \equiv d\left(\mathfrak{q}_{1}\right)$. Clearly $\mathrm{q}_{1}=(x-c, y-d)$. Replacing, if necessary, $x-c$ by $x$ and $y-d$ by $y$, we may without loss of generality assume $q_{1}=(x, y)$. At least one of the elements $x, y$ is not in $\mathfrak{q}_{2}$, say $x \neq 0\left(\mathfrak{q}_{2}\right)$, or equivalently, $v\left(\mathfrak{q}_{1}\right)=v(x)$. Since $v$ is 0 -dimensional and $K$ is algebraically closed there exists a constant $b$ such that $v(y-b x)>0$. Replacing, if necessary, $y-b x$ by $y$, we may without loss of generality assume $v(y)>v(x)$.

With these assumptions on $v(x)$ and $v(y)$, we consider the quadratic transformation $T$ :

$$
x^{\prime}=x, \quad y^{\prime}=y / x ; \quad x=x^{\prime}, \quad y=x^{\prime} y^{\prime},
$$

having at $x=y=0$ a fundamental point, and we denote by $\mathfrak{O}^{\prime}$ the ring of polynomials in $x^{\prime}, y^{\prime}: \mathfrak{D}^{\prime}=K\left[x^{\prime}, y^{\prime}\right]$. $\mathfrak{D}$ is a subring of $\mathfrak{D}^{\prime}$, and moreover $\mathfrak{D}^{\prime} \subset \mathfrak{B}$ since $v\left(y^{\prime}\right)=v(y / x)>0$. Let $\mathfrak{q}_{0}^{\prime}, \mathfrak{q}_{1}^{\prime}, \ldots$ be the Jordan sequence of $v$-ideals in $\mathfrak{D}^{\prime}$ belonging to $v$.

If $\mathfrak{A}$ is any ideal in $\mathfrak{D}$ and if $\mathfrak{D}^{\prime} \mathfrak{A}=x^{\prime h} \mathfrak{X}^{\prime}, \mathfrak{A}^{\prime} \not \equiv 0\left(x^{\prime}\right)$, the ideal $\mathfrak{A}^{\prime}$ is called the transformed ideal of $\mathfrak{A}$ (under the quadratic transformation $T$ ), in symbols: $\mathfrak{A}^{\prime}=T(\mathfrak{A})$. By a theorem of Zariski( $\left.{ }^{4}\right)$, the transform of a v-ideal in $\mathfrak{O}$ for $v$ is a v-ideal in $\mathfrak{D}^{\prime}$ for $v$. We shall have occasion to give another proof of this theorem.

Our principal goal below is to prove that the transform of a simple $v$-ideal is simple. By a simple ideal we mean the following. An arbitrary ideal $\mathfrak{A}$ in $\mathfrak{D}$ is said to be composite if it is the product of two ideals $\mathfrak{B}$, $\mathfrak{\subseteq}$ in $\mathfrak{D}$ different from the unit ideal : $\mathfrak{A}=\mathfrak{B C}, \mathfrak{B} \neq \mathfrak{D}, \mathfrak{E} \neq \mathfrak{O}$. $\mathfrak{A}$ is said to be simple if it is not composite. It is true that if $\mathfrak{A}$ is a v-ideal for $v$ and is composite, $\mathfrak{A}=\mathfrak{B} \mathfrak{C}$, then $\mathfrak{A}$ is also the product of two v-ideals for $v$ different from the unit ideal. In fact, let $\mathfrak{B}^{\prime}, \mathfrak{S}^{\prime}$ be the $v$-ideals in $\mathfrak{O}$ for $v$ such that $v(\mathfrak{B})=v\left(\mathfrak{B}^{\prime}\right), v(\mathfrak{C})=v\left(\mathbb{C}^{\prime}\right)$. Since $\mathfrak{B}$, $\mathfrak{C}$ differ

(4) O. Zariski, ibid., Theorem 4.1. 
from $\mathfrak{D}$ and therefore $\mathfrak{A} \subset \mathfrak{B}, \mathfrak{A} \subset \mathfrak{S}$ properly by a well known theorem, we have $v(\mathfrak{B})>0, v(\mathfrak{C})>0$, whence $v\left(\mathfrak{B}^{\prime}\right)>0, v\left(\mathfrak{S}^{\prime}\right)>0$, and $\mathfrak{B}^{\prime}, \mathfrak{C}^{\prime}$ differ from $\mathfrak{D}$. Since $\mathfrak{B}^{\prime} \supseteq \mathfrak{B}$, $\mathfrak{C}^{\prime} \supseteq \mathfrak{C}$ we have $\mathfrak{B}^{\prime} \mathfrak{C}^{\prime} \supseteq \mathfrak{B} \mathfrak{C}=\mathfrak{A}$. On the other hand, $v(\mathfrak{H})=v(\mathfrak{B} \mathfrak{C})$ $=v\left(\mathfrak{B}^{\prime} \mathfrak{S}^{\prime}\right)$, and since $\mathfrak{A}$ is a $v$-ideal for $v, \mathfrak{A} \supseteq \mathfrak{B}^{\prime} \mathfrak{S}^{\prime}$. Thus $\mathfrak{A}=\mathfrak{B}^{\prime} \mathfrak{S}^{\prime}$. This completes the proof. This remark permits us in proving the simplicity of a given $v$-ideal $\mathfrak{A}$ to restrict the discussion, first, to any valuation $v$ for which $\mathfrak{A}$ is a $v$-ideal, and second, to the ideals in $\mathfrak{D}$ which are $v$-ideals for $v$.

4. The valuation given by $y=x^{\tau}$. Let $\mathfrak{D}=K[x, y]$ be a polynomial ring in two indeterminates $x, y$ over the field $\left(^{5}\right) K$, and let $\Sigma$ be the quotient field of $\mathcal{D}$. Let $\tau$ be an arbitrary irrational number greater than zero.

There exists one and only one valuation $v$ of $\Sigma$ over $K$ such that $v(x)=1$ and $v(y)=\tau . v$ is necessarily 0 -dimensional. The value group of this valuation is the additive group of real numbers of the form $a+b \tau$ where $a$ and $b$ are rational integers. (See, for example, Saunders MacLane and O. F. G. Schilling, Zero-dimensional branches of rank one on algebraic varieties, construction I, Ann. of Math. vol. 40 (1939).) We shall say that the valuation $v$ is given by $y=x^{\tau}$, or that $y=x^{\tau}$ is the valuation $v$.

Consider the valuation $v$ given by $y=x^{\tau}, \tau$ an arbitrary positive irrational number. Since $v(x)>0$ and $v(y)>0$, the ring $\mathfrak{D}=K[x, y]$ is contained in the valuation ring $\mathfrak{B}$ of $v$. Let $q_{0}, q_{1}, q_{2}, \cdots$ be the Jordan sequence of $v$-ideals for $v$. Here $q_{0}=\mathfrak{D}, q_{1}=p=(x, y)$.

From the definition of $v$ every $v$-ideal $\mathfrak{q}_{i}$ has value $m_{i}+n_{i} \tau, m_{i}, n_{i}$ nonnegative integers. Conversely, every number $m+n \tau, m, n$ non-negative integers, is the value of one of the ideals $\mathfrak{q}_{i}$, namely that one which contains all the elements in $\mathfrak{D}$ of value equal to or greater than $v\left(x^{m} y^{n}\right)$. Hence the sequence $v\left(\mathfrak{q}_{0}\right), v\left(\mathfrak{q}_{1}\right), v\left(\mathfrak{q}_{2}\right), \cdots$ coincides with the set of numbers $\{g+h \tau\}$, $g$ and $h$ non-negative integers, ordered according to magnitude.

THEOREM 1. Every $v$-ideal for the valuation given by $y=x^{\tau}$ has a basis consisting of monomials $x^{m} y^{n}$.

Proof. Let $q$ be a $v$-ideal for $v$, and let $g(x, y) \in q$. If $g(x, y)=\sum c_{m n} x^{m} y^{n}$, $c_{m n} \in K, c_{m n} \neq 0$, then $v(g)=\min \left(v\left(x^{m} y^{n}\right)\right)$, by definition; that is, $v\left(x^{m} y^{n}\right) \geqq v(g)$ $\geqq v(\mathfrak{q})$ for every term $c_{m n} x^{m} y^{n}$ of $g(x, y)$, whence $x^{m} y^{n} \in q$ for every such term. Thus a basis for $q$ can be replaced by all the various terms in the elements of the basis. This completes the proof.

Corollary. Let $m_{1}+n_{1} \tau, m_{2}+n_{2} \tau, \cdots$ be the set of positive numbers $\{g+h \tau\}, g, h$ non-negative integers, ordered according to magnitude. The ideals $\mathfrak{q}_{1}, \mathfrak{q}_{2}, \cdots$ can be described in the following manner: $\mathfrak{q}_{i}$ has a basis consisting of the elements $x^{m(k)} y^{n(k)}, k \geqq i$, where $m(k)=m_{k}$, a notation used later also.

THEOREM 2.1. If $v\left(\mathfrak{q}_{i}\right)=m+n \tau, v\left(\mathfrak{q}_{j}\right)=m, v\left(\mathfrak{q}_{k}\right)=n \tau$ then $\mathfrak{q}_{i}=\mathfrak{q}_{j} \mathfrak{q}_{k}$.

( $\left.{ }^{(}\right) K$ need not be algebraically closed in this section. 
THEOREM 2.2. If $v\left(\mathfrak{q}_{i}\right)=s \tau, v\left(\mathfrak{q}_{i+1}\right)=m+n \tau$ and if $m n \neq 0$, then $s>n$ and $\mathfrak{q}_{i}=\mathfrak{q}_{j} \mathfrak{q}_{k}$, where $v\left(\mathfrak{q}_{j}\right)=n \tau, v\left(\mathfrak{q}_{k}\right)=(s-n) \tau$.

THEOREM 2.3. If $v\left(\mathfrak{q}_{i}\right)=r, v\left(\mathfrak{q}_{i+1}\right)=m+n \tau$ and if $m n \neq 0$, then $r>m$ and $\mathfrak{q}_{i}=\mathfrak{q}_{j} \mathfrak{q}_{k}$, where $v\left(\mathfrak{q}_{j}\right)=m, v\left(\mathfrak{q}_{k}\right)=r-m$.

Proof. 2.1. Clearly $\mathfrak{q}_{j} \mathfrak{q}_{k} \subseteq \mathfrak{q}_{i}$. Conversely, let $x^{m^{\prime}} y^{n^{\prime}} \in \mathfrak{q}_{i}$. Since $m^{\prime}+n^{\prime} \tau$ $\geqq m+n \tau$, either $n^{\prime} \geqq n$ or $m^{\prime} \geqq m$. In the first case,

$$
x^{m^{\prime}} y^{n^{\prime}}=x^{m^{\prime}} y^{n^{\prime}-n} \cdot y^{n} \in \mathfrak{q}_{j} \mathfrak{q}_{k}
$$

since $x^{m^{\prime}} y^{n^{\prime}-n} \in \mathfrak{q}_{j}$ (in view of $m^{\prime}+\left(n^{\prime}-n\right) \tau \geqq m$ ) and $y^{n} \in \mathfrak{q}_{k}$. In the second case

$$
x^{m^{\prime}} y^{n^{\prime}}=x^{m} \cdot x^{m^{\prime}-m} y^{n^{\prime}} \in \mathfrak{q}_{j} \mathfrak{q}_{k}
$$

since $x^{m} \in \mathfrak{q}_{j}, x^{m^{\prime}-m} y^{n^{\prime}} \in q_{k}$. This completes the proof.

2.2. We cannot have $n \geqq s$, for $n \geqq s$ together with $m \neq 0$ implies $m+n \tau-s \tau$ $\geqq 1$, whence there exists an integer between the irrational numbers $s \tau$ and $m+n \tau$. (Note that $s \neq 0$, for if $s=0$ then $v\left(\mathfrak{q}_{i+1}\right)=1$ or $\tau$.) This contradicts the fact that $s \tau$ and $m+n \tau$ are successive in the ordered set of numbers $\{g+h \tau\}, g, h$ non-negative integers. Hence $s>n$, and the ideals $\mathfrak{q}_{j}, \mathfrak{q}_{k}$ exist. Clearly $\mathfrak{q}_{j} \mathfrak{q}_{k} \subseteq \mathfrak{q}_{i}$. Conversely, let $\mathfrak{q}_{i}=\left(y^{s}, x^{m} y^{n}, x^{m^{\prime}} y^{n^{\prime}}, \cdots\right)$. Then clearly $y^{s}=y^{n} y^{s-n} \in \mathfrak{q}_{j} \mathfrak{q}_{k}$ and $x^{m} y^{n} \in \mathfrak{q}_{j} \mathfrak{q}_{k}$ since $m>(s-n) \tau$, so that $x^{m} \in \mathfrak{q}_{k}$. As for $x^{m^{\prime}} y^{n^{\prime}}$, either $n^{\prime} \geqq m$ or $n^{\prime} \geqq n$, since $m^{\prime}+n^{\prime} \tau \geqq m+n \tau$. Hence if we write $x^{m^{\prime}} y^{n^{\prime}}$ in the form $x^{m} \cdot x^{m^{\prime}-m} y^{n^{\prime}}$ or in the form $x^{m^{\prime}} y^{n^{\prime}-n} \cdot y^{n}$, we conclude in either case that $x^{m^{\prime}} y^{n^{\prime}} \in \mathfrak{q}_{j} \mathfrak{q}_{k}$. This completes the proof.

2.3. This case is really not different from the case 2.2 . In fact, consider the order preserving automorphism of the additive group of real numbers given by: $x \rightarrow x / \tau$. If we replace the value group of $v$ by the isomorphic group obtained under this automorphism, the statement and proof of 2.3 are the same as those of 2.2. This completes the proof.

THEOREM 3. Let $h$ be the greatest integer less than $\tau$ if $\tau>1$, or the greatest integer less than $1 / \tau$ if $\tau<1$. If $i \leqq h$ then $\mathfrak{q}_{i}$ is simple. If $i \geqq h$ and if $\mathfrak{q}_{i}$ is simple, then of the two numbers $v\left(\mathfrak{q}_{i}\right), v\left(\mathfrak{q}_{i+1}\right)$ one is an integer, the other an integral multiple of $\tau$.

Proof. If $\tau>1$ then $v\left(\mathfrak{q}_{1}\right)=1, v\left(\mathfrak{q}_{2}\right)=2, \cdots, v\left(\mathfrak{q}_{h}\right)=h, v\left(\mathfrak{q}_{h+1}\right)=\tau$. If $\tau<1$ then $v\left(\mathfrak{q}_{1}\right)=\tau, v\left(\mathfrak{q}_{2}\right)=2 \tau, \cdots, v\left(\mathfrak{q}_{h}\right)=h \tau, v\left(\mathfrak{q}_{h+1}\right)=1$. In the first case $y \in \mathfrak{q}_{i}$, $i=1,2, \cdots, h$; in the second case $x \in q_{i}, i=1, \cdots, h$. In either case the ideals $q_{1}, q_{2}, \cdots, q_{h}$ must be simple because each element of the product of two ideals which are different from the unit ideal must have a leading form of degree at least 2 .

If, now, $i \geqq h$ and $v\left(\mathfrak{q}_{i}\right)=m+n \tau, m \neq 0, n \neq 0$, then Theorem 2.1 proves that $q_{i}$ is not simple.

If $i \geqq h, v\left(\mathfrak{q}_{i}\right)=s \tau$ and $v\left(\mathfrak{q}_{i+1}\right)=m+n \tau$ and $n \neq 0$, then also $m \neq 0$. In fact, if $m=0$ then clearly $n=s+1$. If $\tau>1$, then $v\left(\mathfrak{q}_{i+1}\right)-v\left(\mathfrak{q}_{i}\right)=\tau>1$, whence there 
is an integer between $v\left(\mathfrak{q}_{i}\right)$ and $v\left(\mathfrak{q}_{i+1}\right):$ this is a contradiction. If $\tau<1$ then $s \tau>1$, since $i \geqq h$ and $i=h$ is excluded because $v\left(\mathfrak{q}_{i+1}\right)=(s+1) \tau \neq 1$. If $k$ is the greatest integer less than $(s \tau-1) / \tau+1$, that is,

$$
(s \tau-1) / \tau<k<(s \tau-1) / \tau+1, k \text { an integer, }
$$

then $s \tau<1+k \tau<(s+1) \tau ; k>0$ since $s \tau-1>0$. This is a contradiction. Hence $m \neq 0$. By Theorem 3.2, then, $q_{i}$ is not simple.

If $i \geqq h, v\left(\mathfrak{q}_{i}\right)=r$ and $v\left(\mathfrak{q}_{i+1}\right)=m+n \tau$ and $m \neq 0$ then similarly $q_{i}$ is not simple. In fact, this case is not really different from the one immediately above, as may be seen by the argument given in 2.3. This completes the proof.

We wish now to prove the converse of Theorem 3. For this we need two lemmas.

Let $m_{1}+1 /\left(m_{2}+1 /\left(m_{3}+\cdots\right.\right.$ be the continued fraction expansion of $\tau$. Consider the sequence of numbers: $m_{1}, m_{1}+1 / 1, m_{1}+1 / 2, \cdots, m_{1}+1 / m_{2}$, $m_{1}+1 /\left(m_{2}+1 / 1, \cdots, m_{1}+1 /\left(m_{2}+1 / m_{3}, \cdots\right.\right.$ Of these, the numbers $m_{1}$, $m_{1}+1 / m_{2}, m_{1}+1 /\left(m_{2}+1 / m_{3}, \cdots\right.$ are known as approximants, the others as quasi approximants $\left(^{6}\right)$, to $\tau$.

Lemma 1. If the two numbers $r, s \tau$ (or $s \tau, r$ ), $r$, s positive integers, are successive in the set of numbers $\{g+h \tau\}, g, h$ non-negative integers, ordered according to magnitude, then $(r, s)=1$ and $r / s$ is an approximant or quasi approximant to $\tau$ (and therefore $s / r$ is also an approximant or quasi approximant to $1 / \tau$ ). Conversely, if $r / s, r, s$ positive integers with $(r, s)=1$, is an approximant or quasi approximant to $\tau$ and $s / r$ is an approximant or quasi approximant to $1 / \tau$ then $r, s \tau$ (or $s \tau, r)$ are successive in the set of numbers $\{g+h \tau\}$.

Proof. Let $r, s \tau$ (or $s \tau, r$ ) be successive in the ordered set of numbers $\{g+h \tau\}$. From the symmetry of the lemma, we need consider only one case, say the case $r<s \tau$. A theorem of Lagrange and its converse $\left({ }^{7}\right)$ state that $r / s$ is an approximant or quasi approximant to $\tau$ if and only if $n>s$ for every rational number $m / n, m, n$ positive integers such that $r / s<m / n<\tau$. Now, if $r / s<m / n<\tau, m, n$ positive integers and $n \leqq s$, then

$$
s \tau-r>(s / n)(n \tau-m) \geqq n \tau-m>0,
$$

whence $r<m+(s-n) \tau<s \tau$, which contradicts the assumption that $r$ and $s \tau$ are successive in the set $\{g+h \tau\}$. Hence $n>s$ and $r / s$ is an approximant or quasi approximant to $\tau$.

Conversely, suppose $(r, s)=1, r, s$ positive integers, and that $r<u+v \tau<s \tau$ for some non-negative integers $u, v$. If $v \neq 0$ then either $(r-u) / v>r / s$ or $u(s-v)>r / s$, for $(r-u) / v \leqq r / s$ and $u /(s-v) \leqq r / s$ imply $r s-u s \leqq r v$ and $u s \leqq r s-r v$, whence $u s=r s-r v$, and $r / s=u /(s-v)$ which contradicts the assumption $(r, s)=1$. Moreover $(r-u) / v$ and $u /(s-v)$ are both less than $\tau$.

(8) Perron, Nebennäherungsbrüche.

( ${ }^{7}$ See O. Perron, Die Lehre von den Kettenbriuchen, p. 38, Theorems 20 and 21. 
Hence there exists a rational number $m / n$ (either $(r-u) / v$ or $u /(s-v)$ ) with $0<n \leqq s$ such that $r / s<m / n<\tau$. The same is true even if $v=0$; namely $m / n=u / s$. By the theorem mentioned above, $r / s$ is therefore neither an approximant nor a quasi approximant to $\tau$. This completes the proof.

LeMma 2. If $m_{1}+n_{1} \tau, m_{2}+n_{2} \tau, \cdots, m_{k}+n_{k} \tau, r$, s $\tau$ is an initial segment of the ordered set of positive numbers $\{g+h \tau\}, g, h$ non-negative integers, then there exists an irrational number $\tau^{\prime}>0$ such that $m_{1}+n_{1} \tau^{\prime}, m_{2}+n_{2} \tau^{\prime}, \cdots, m_{k}$ $+n_{k} \tau^{\prime}, s \tau^{\prime}, r$ is an initial segment of the ordered set of positive numbers $\left\{g+h \tau^{\prime}\right\}$, $g, h$ non-negative integers. A similar statement holds for an initial segment $m_{1}+n_{1} \tau, m_{2}+n_{2} \tau, \cdots, m_{k}+n_{k} \tau, s \tau, r$.

Proof. If $s=1$, then $n_{1}=n_{2}=\cdots=n_{k}=0$ and any irrational number $\tau^{\prime}$ between $r-1 / 2$ and $r$ satisfies the lemma. If $r=1$, then any irrational number $\tau^{\prime}$ between $1 /(s+1)$ and $1 / s$ satisfies the lemma. If $s \neq 1, r \neq 1$ then $r / s$ and $s / r$ are not integers, since $(r, s)=1$. Let $r / s$ have a continued fraction expansion $b_{1}+1 /\left(b_{2}+1 /\left(b_{3}+\cdots+1 /\left(b_{l}\right.\right.\right.$ with $b_{l}>1$. It is well known that if $\sigma$ is an arbitrary irrational number greater than 1 then $b_{1}+1 /\left(b_{2}+\ldots\right.$ $+1 /\left(\left(b_{l}-1\right)+1 /\left(1+1 /\left(\sigma\right.\right.\right.$ and $b_{1}+1 /\left(b_{2}+\cdots+1 /\left(b_{l}+1 /(\sigma\right.\right.$ are irrational numbers having $r / s$ as an approximant, of which one is larger, the other smaller than $r / s$. Thus there exist irrational numbers $\tau^{\prime}<r / s$ having $r / s$ as an approximant: $s / r$ will also be an approximant to $1 / \tau^{\prime}$. Let $\tau^{\prime}(<r / s)$ be any irrational number having $r / s$ as an approximant or quasi approximant. We assert that $\tau^{\prime}$ satisfies the lemma. In fact, by Lemma $1, s \tau^{\prime}$ and $r$ are successive in the ordered set of numbers $\left\{g+h \tau^{\prime}\right\}$. Moreover, if $u+v \tau^{\prime}<s \tau^{\prime}, u, v$ non-negative integers, then $v<s$, whence $u+v \tau<s \tau$ since $\tau^{\prime}<\tau$. Hence $u+v \tau \leqq r$; equality is excluded since $u+v \tau=r$ implies $v=0, u=r>s \tau^{\prime}$. Thus $u+v \tau<r$. Similarly, if $u+v \tau<r, u, v$ non-negative integers, then $u+v \tau^{\prime}<r$ since $\tau^{\prime}<\tau$. Hence $u+v \tau^{\prime} \leqq s \tau^{\prime}$; equality is excluded, since $u+v \tau^{\prime}=s \tau^{\prime} \mathrm{im}$ plies $u=0, v \tau=s \tau<r$. It remains to prove that $m_{i}+n_{i} \tau^{\prime}<m_{i+1}+n_{i+1} \tau^{\prime}$, $i=1, \cdots, k-1$. If $n_{i+1}=n_{i}$ this is clear; if $n_{i} \neq n_{i+1}$, then $\left(m_{i}-m_{i+1}\right) /\left(n_{i+1}-n_{i}\right)$ $>\tau$ or $<\tau$ according as $n_{i+1}<n_{i}$ or $n_{i+1}>n_{i}$. If $\left(m_{i}-m_{i+1}\right) /\left(n_{i+1}-n_{i}\right)>\tau\left(>\tau^{\prime}\right)$ then $n_{i+1}-n_{i}<0$ and $m_{i}+n_{i} \tau^{\prime}<m_{i+1}+n_{i+1} \tau^{\prime}$. If $\left(m_{i}-m_{i+1}\right) /\left(n_{i+1}-n_{i}\right)<\tau$ then $\left(m_{i}-m_{i+1}\right) /\left(n_{i+1}-n_{i}\right)<r / s$ also since $0<n_{i+1}-n_{i}<s$ and $r / s$ is an approximant or quasi approximant to $\tau$. But then, again because $0<n_{i+1}-n_{i}<s$ and $r / s$ is an approximant or quasi approximant to $\tau^{\prime},\left(m_{i}-m_{i+1}\right) /\left(n_{i+1}-n_{i}\right)>\tau^{\prime}$ is impossible, that is, $\left(m_{i}-m_{i+1}\right) /\left(n_{i+1}-n_{i}\right)<\tau^{\prime}$ and $m_{i}+n_{i} \tau^{\prime}<m_{i+1}+n_{i+1} \tau^{\prime}$ follows. This completes the proof.

THEOREM 4. If of the numbers $v\left(\mathfrak{q}_{i}\right), v\left(\mathfrak{q}_{i+1}\right)$ one is an integer, the other an integral multiple of $\tau$, then $\mathrm{q}_{i}$ is simple.

Proof. The case $i=0$ is trivial. If $i>0$ then $v\left(\mathfrak{q}_{i}\right)=r, v\left(\mathfrak{q}_{i+1}\right)=s \tau$ or $v\left(\mathfrak{q}_{i}\right)=s \tau, v\left(\mathfrak{q}_{i+1}\right)=r, r, s$ positive integers. As both cases are similar, we consider for the sake of brevity only the case $v\left(\mathfrak{q}_{i}\right)=s \tau$. Consider the sequence 
$v\left(\mathfrak{q}_{1}\right), v\left(\mathfrak{q}_{2}\right), \cdots$, that is, the sequence:

$$
m_{1}+n_{1} \tau, m_{2}+n_{2} \tau, \cdots, m_{i-1}+n_{i-1} \tau, s \tau, r, \cdots,
$$

where $v\left(\mathfrak{q}_{j}\right)=m_{j}+n_{j} \tau$. By Lemma 2 there exists an irrational number $\tau^{\prime}$ such that

$$
m_{1}+n_{1} \tau^{\prime}, m_{2}+n_{2} \tau^{\prime}, \cdots, m_{i-1}+n_{i-1} \tau^{\prime}, r, s \tau^{\prime}
$$

is an initial segment of the set of positive numbers $\left\{g+h \tau^{\prime}\right\}, g, h$ non-negative in tegers, ordered according to magnitude. Consider ine valuation $v^{\prime}$ given by $y=x^{\tau^{\prime}}$, and let $q_{1}^{\prime}=(x, y), q_{2}^{\prime}, \ldots$ be the Jordan sequence of (0-dimensional) valuation ideals in $\mathfrak{D}=K[x, y]$ for the valuation $v^{\prime}$. By the corollary to Theorem 1, $\mathfrak{q}_{1}=\mathfrak{q}_{1}^{\prime}, \mathfrak{q}_{2}=\mathfrak{q}_{2}^{\prime}, \cdots, \mathfrak{q}_{i}=\mathfrak{q}_{i}^{\prime}$. The assumption $\mathfrak{q}_{i}=\mathfrak{q}_{j} \mathfrak{q}_{k}, j<i$, $k<i$, now leads to a contradiction. In fact, $\left(m_{j}+n_{j} \tau\right)+\left(m_{k}+n_{k} \tau\right)=s \tau$, whence $m_{j}+m_{k}=0$. Since $m_{j}, m_{k}$ are non-negative, $m_{j}=m_{k}=0$, whence $v\left(\mathfrak{q}_{j}\right)=n_{j} \tau$, $v\left(\mathfrak{q}_{k}\right)=n_{k} \tau$. But the $j$ th and $k$ th numbers in the sequence $m_{1}+n_{1} \tau^{\prime}, m_{2}+n_{2} \tau^{\prime}$, $\cdots, m_{i-1}+n_{i-1} \tau^{\prime}, r, s \tau^{\prime}$ are $n_{j} \tau^{\prime}, n_{k} \tau^{\prime}$, whence $v^{\prime}\left(\mathfrak{q}_{j}\right)=n_{j} \tau^{\prime}, v^{\prime}\left(\mathfrak{q}_{k}\right)=n_{k} \tau^{\prime}$, whence $v^{\prime}\left(\mathfrak{q}_{i}\right)=v^{\prime}\left(\mathfrak{q}_{j} \mathfrak{q}_{k}\right)=\left(n_{j}+n_{k}\right) \tau^{\prime}=s \tau^{\prime}$; this is a contradiction. This completes the proof.

Let $m_{1}+1 /\left(m_{1}+1 /\left(m_{3}+\cdots\right.\right.$ be the continued fraction expansion for $\tau$; and consider the sequence: $a_{0}=0, a_{1}=1, a_{2}=2, \cdots, a_{m(1)}=m_{1}, a_{m(1)+1}$ $=m_{1}+1 / 1, a_{m(1)+2}=m_{1}+1 / 2, \cdots, a_{m(1)+m(2)}=m_{1}+1 / m_{2}, \cdots$ Let $\mathfrak{B}_{0}, \mathfrak{B}_{1}$, $\mathfrak{B}_{2}, \cdots$ be the sequence of simple $v$-ideals for the valuation $y=x^{\tau}$ in $K[x ; y]$, $\mathfrak{B}_{i} \supset \mathfrak{B}_{i+1}, i \geqq 0$.

COROLLARY. There is a 1-1 correspondence $\mathfrak{B}_{i} \rightleftarrows a_{i}$ between the simple v-ideals $\mathfrak{B}_{i}$ and the $a_{i}, i \geqq 0$. The correspondence is such that if $a_{i}=r / s, r, s$ non-negative integers with $(r, s)=1$, then $v\left(\mathfrak{P}_{i}\right)=\min (r, s \tau)$.

Proof. Consider first the case $\tau>1$, or equivalently, $m_{1} \geqq 1$. By Theorem $3, v\left(\mathfrak{B}_{i}\right)=i=\min (i, \tau), i=0,1, \cdots, m_{1}$. By Theorems 3 and 4 , for $k \geqq m_{1}, \mathfrak{q}_{k}$ is simple if and only if either $v\left(\mathfrak{q}_{k}\right)=r, v\left(\mathfrak{q}_{k+1}\right)=s \tau$ or $v\left(\mathfrak{q}_{k}\right)=s \tau$, $v\left(\mathfrak{q}_{k+1}\right)=r, r, s$ positive integers. By Lemma $1, r / s$ is an approximant or quasi approximant to $\tau$. Conversely, let $r / s, r, s$ positive integers with $(r, s)=1$, be an approximant or quasi approximant to $\tau$. Since $1 / \tau<1, s / r$ is certainly also an approximant or quasi approximant to $1 / \tau$. Hence $r, s \tau$ or $s \tau, r$ are successive in the ordered set $\{g+h \tau\}, g, h$ non-negative integers; that is, if $v\left(\mathfrak{q}_{k}\right)=\min (r, s \tau)$ then $v\left(\mathfrak{q}_{k+1}\right)=\max (r, s \tau)$ and $\mathfrak{q}_{k}$ is simple by Theorem 4 . We make $\mathfrak{q}_{k}$ and $r / s$ correspond, and it remains to show that $\mathfrak{B}_{i} \rightleftarrows a_{i}$. It is well known that if $a_{i}=r_{i} / s_{i}, r_{i}, s_{i}$ positive integers with $\left(r_{i}, s_{i}\right)=1$, then $r_{i}<r_{i+1}$. Since the set $\{g+h \tau\}$ is ordered according to magnitude, it is clear that the correspondence could not be other than $\mathfrak{B}_{i} \rightleftarrows a_{i}$.

Consider now the case $\tau<1$, or equivalently, $m_{1}=0$. Here $a_{0}=0$, $a_{1}=0+1 / 1, \cdots$. The valuation $y=x^{\tau}$ is also given by $x=y^{1 / \tau}$. Let $b_{0}=0, b_{1}, b_{2}, \cdots$ be the sequence for $1 / \tau$ corresponding to the sequence $\left\{a_{i}\right\}$ for $\tau$. Clearly $b_{i}=1 / a_{i}$ for $i>0$; if $a_{i}=r_{i} / s_{i}, r_{i}, s_{i}$ positive integers, 
$\left(r_{i}, s_{i}\right)=1$, then $b_{i}=s_{i} / r_{i}$. By the case $\tau>1, \mathfrak{B}_{i} \rightleftarrows b_{i}$ and the correspondence is such that $v\left(\mathfrak{B}_{i}\right)=\min \left(s_{i} v(y),\left(r_{i} / \tau\right) v(y)\right)$. Since $v(y)=\tau, v\left(\mathfrak{B}_{i}\right)=\min \left(s_{i} \tau, r_{i}\right)$. This completes the proof.

Theorems 3 and 4 enable us to prove our principal theorem for the valuation $y=x^{r}$; that is, that the transform of a simple $v$-ideal under the transformation $T$ (see $\S 3$ ) is simple. Here we assume that $\tau>1$, whence $v(y)>v(x)$. $T$ is then the transformation:

$$
T: \quad x^{\prime}=x, \quad y^{\prime}=y / x ; \quad x=x^{\prime}, \quad y=x^{\prime} y^{\prime} .
$$

As above we denote the ring $K\left[x^{\prime}, y^{\prime}\right]=K[x, y / x]$ by $\mathfrak{D}^{\prime}$, and the $v$-ideals in $\mathfrak{D}^{\prime}$ for $v$ by $q_{0}^{\prime}, q_{1}^{\prime}, q_{2}^{\prime}, \ldots$. Consider the valuations given by $y=x^{\tau}$ and $y^{\prime}=x^{\prime r-1}$. Since in both the value of $x$ is 1 and the value of $y^{\prime}$ is $\tau-1$, they are, in fact, the same valuation: that is, the valuation $y=x^{\tau}$ is also given by $y^{\prime}=x^{\prime r-1}$.

Let $\mathfrak{P}_{0}=q_{0}, \mathfrak{B}_{1}=q_{1}, \mathfrak{P}_{2}, \cdots$ be the simple $v$-ideals for $v$ in $\mathfrak{D}$ and let $\mathfrak{B}_{0}^{\prime}=q_{0}^{\prime}, \mathfrak{P}_{1}^{\prime}=\mathfrak{q}_{1}^{\prime}, \mathfrak{P}_{2}^{\prime}, \cdots$ be the simple $v$-ideals for $v$ in $\mathfrak{D}^{\prime}$.

Theorem 5. The transform of the ith simple v-ideal $\mathfrak{B}_{i}, i>0$, is $\mathfrak{B}_{i-1}^{\prime}$.

Proof. Let $m_{1}+1 /\left(m_{2}+1 /\left(m_{3}+\cdots\right.\right.$ be the continued fraction expansion for $\tau ; m_{1} \geqq 1$ since $\tau>1$. Let $a_{1}=1, a_{2}=2, \cdots, a_{m(1)}=m_{1}, a_{m(1)+1}=m_{1}+1 / 1$, $a_{m(1)+2}=m_{1}+1 / 2, \cdots, a_{m(1)+m(2)}=m_{1}+1 / m_{2}, \cdots$. By the preceding corollary there is a $1-1$ correspondence between the numbers $a_{i}$ and the ideals $\mathfrak{P}_{i}$; the correspondence is such that if $a_{i}=r / s, r, s$ positive integers with $(r, s)=1$, then $v\left(\mathfrak{B}_{i}\right)=\min (r, s \tau)$. Let $\left\{a_{i}^{\prime}\right\}$ represent the corresponding numbers for $\tau-1(>0)$. Clearly $a_{i}^{\prime}+1=a_{i+1}$.

For $i=1,2, \cdots, m_{1}$ the ideal $\mathfrak{B}_{i}=\mathfrak{q}_{i}$ is simple and contains the element $y$. Clearly $\mathfrak{D} \mathfrak{q}_{i} \equiv 0\left(x^{\prime}\right)$. Moreover $y=y^{\prime} x^{\prime} \in \mathfrak{q}_{i}$ so that $\mathfrak{D} \mathfrak{q}_{i} \neq \equiv\left(x^{\prime 2}\right)$. Hence, by the definition of $T, v\left(T\left(\mathfrak{q}_{i}\right)\right)=v\left(\mathfrak{q}_{i}\right)-1=i-1$, whence $T\left(\mathfrak{q}_{i}\right) \subseteq \mathfrak{B}_{i-1}^{\prime}$. By the theorem of Zariski mentioned in $\S 3, T\left(\mathfrak{q}_{i}\right)$ is a $v$-ideal for $v$, and since $v\left(T\left(\mathfrak{q}_{i}\right)\right)$ $=v\left(\mathfrak{P}_{i-1}^{\prime}\right)$ we have $T\left(\mathfrak{q}_{i}\right)=\mathfrak{B}_{i-1}^{\prime}$.

In the special case with which we are dealing here it is also possible to prove this result by a direct and simple calculation. In fact, clearly $\mathfrak{B}_{i-1}^{\prime}=q_{i-1}^{\prime}$ $=\left(y^{\prime}, x^{\prime-1}\right)$. The element $y^{\prime}$ arises from $y \in q_{i}$, and the element $x^{\prime i-1}$ arises from $x^{i} \in \mathfrak{q}_{i}$, whence, together with $T\left(\mathfrak{q}_{i}\right) \subseteq \mathfrak{B}_{i-1}^{\prime}$, we have $T\left(\mathfrak{q}_{i}\right)=\mathfrak{B}_{i_{-1}^{\prime}}$. Thus Theorem 5 is true at least for $i=1,2, \cdots, m_{1}$.

For $i>m_{1}, v\left(\mathfrak{B}_{i}\right)=r, v(\mathfrak{q})=s \tau$ or $v\left(\mathfrak{B}_{i}\right)=s \tau, v(\mathfrak{q})=r, r, s$ positive integers, where $q$ is the element of the sequence $q_{1}, q_{2}, \ldots$ immediately following $\mathfrak{B}_{i}$. The two cases are not really different: in fact, let $m_{1}+n_{1} \tau, m_{2}+n_{2} \tau, \cdots, m_{k}$ $+n_{k} \tau, r, s \tau$ be an initial segment of the ordered set of positive numbers $\{g+h \tau\}, g, h$ non-negative integers. By Lemma 2 of Theorem 4, there exists an irrational number $\tau^{\prime}>0$ such that $m_{1}+n_{1} \tau^{\prime}, m_{2}+n_{2} \tau^{\prime}, \cdots, m_{k}+n_{k} \tau^{\prime}$, $s \tau^{\prime}, r$ is an initial segment of the ordered set $\left\{g+h \tau^{\prime}\right\}, g, h$ non-negative integers. By the description of the $v$-ideals for $y=x^{\tau}$ given in the corollary to 
Theorem 1 , we see that $q_{1}, q_{2}, \cdots, \mathfrak{B}_{i}$ are $v$-ideals for the valuation $y=x^{\boldsymbol{r}^{\prime}}$ : and in this valuation the value of $\mathfrak{B}_{i}$ is $s \tau^{\prime}$. Moreover, since $i>m_{1}$ the numbers $m_{1}, \tau$ are among the first $k$ numbers of the set $\{g+h \tau\}$, whence also $m_{1}, \tau^{\prime}$ are among the first $k$ of $\left\{g+h \tau^{\prime}\right\}$, whence $m_{1}<\tau^{\prime}$. Thus by changing from $\tau$ to $\tau^{\prime}$ we retain the assumption $\tau>1$. Therefore without loss of generality we need consider only the case $v\left(\mathfrak{P}_{i}\right)=s \tau, v(\mathfrak{q})=r$.

Now, $a_{i}=r / s$, whence $a_{i-1}^{\prime}=(r-s) / s$ (and clearly $\left.(r-s, s)=1\right)$. Since $s \tau<r$, we have $s \tau^{\prime}=s(\tau-1)<r-s$; whence $v\left(\mathfrak{B}_{i-1}^{\prime}\right)=s \tau$. On the other hand, $\mathfrak{P}_{i}$ has a basis consisting of monomials $x^{m} y^{n}$ with $m+n \tau \geqq s \tau$; since $\tau>1$, $m+n \geqq s$, whence $\mathfrak{O M}_{i} \equiv 0\left(x^{\prime s}\right)$ and $T\left(\mathfrak{B}_{i}\right) \not \equiv 0\left(x^{\prime s+1}\right)$ since $\mathfrak{B}_{i} \supset y^{s}=y^{\prime s} x^{\prime s}$ $\not \equiv 0\left(x^{\prime s+1}\right)$. By definition, then, $v\left(T\left(\mathfrak{B}_{i}\right)\right)=v\left(\mathfrak{B}_{i}\right)-s=s(\tau-1)=s \tau^{\prime}=v\left(\mathfrak{P}_{i-1}^{\prime}\right)$. Applying the fact that $T\left(\mathfrak{B}_{i}\right)$ is a $v$-ideal for $v$, we have $T\left(\mathfrak{B}_{i}\right)=\mathfrak{B}_{i-1}^{\prime}$. This completes the proof.

5. The general case; reduction to rational rank 2 valuations. Let $\gamma_{1}, \gamma_{2}, \cdots$ be a sequence of positive rational numbers. Let $\Delta_{0}$ be the additive group of integers. For a given $i \geqq 1$, we consider the additive abelian group $G_{i}$ generated by $1, \gamma_{1}, \cdots, \gamma_{i}$, that is, the set of numbers of the form $m+n_{1} \gamma_{1}$ $+\cdots+n_{i} \gamma_{i}, m, n_{j}$ integers. In this sequence of groups let $\Delta_{j}$ be the $j$ th group $G$ containing its predecessor properly. Let $\Gamma_{0}$ be the set of nonnegative integers, $\Gamma_{j}$ the set of numbers in $\Delta_{j}$ which are of the form $m+n_{1} \gamma_{i(1)}+\cdots+n_{j} \gamma_{i(j)}$, where $m, n_{i}$ are non-negative integers. We denote the numbers $\gamma_{i(1)}, \gamma_{i(2)}, \cdots$ by $\delta_{1}, \delta_{2}, \cdots$.

Let $\delta_{k}=r_{k} / s_{k}$, where $r_{k}, s_{k}$ are positive integers and $\left(r_{k}, s_{k}\right)=1$; let $t_{0}=1$, $t_{k}=1$. .c.m. $\left(s_{1}, \cdots, s_{k}\right)$; and let $d_{k}=t_{k} / t_{k-1}$. Since $\Delta_{k}$ consists of all the numbers $m / t_{k}, m$ an integer, it is clear that $d_{k}$ is the smallest positive integer such that $d_{k} \delta_{k} \in \Delta_{k-1}$.

Lemma 3. If for a given $j, \delta_{l}>d_{l-1} \delta_{l-1}$ for all $l \leqq j$, then

$\left(\mathrm{a}_{j}\right) k / t_{j} \geqq d_{j} \delta_{j}, k$ an integer, $j \geqq 1$, implies $k / t_{j} \in \Gamma_{j}$,

$\left(\mathrm{b}_{j}\right) d_{j} \delta_{j} \in \Gamma_{j-1}, j \geqq 1$.

Proof. The lemma may be vacuously true, in fact, is vacuous if and only if the $\gamma_{i}$ are integers. We suppose this not to be the case: $\delta_{1}=\gamma_{i(1)}=r_{1} / s_{1}$ is then the first non-integer in the sequence $\gamma_{1}, \gamma_{2}, \cdots$.

For $j=1$ we have $t_{j}=t_{1}=s_{1}, t_{j-1}=t_{0}=1$, so that $d_{1}=s_{1}$; whence (b) holds for $j=1$. For $j=1,\left(\mathrm{a}_{1}\right)$ becomes: if $k / s_{1} \geqq s_{1} \cdot r_{1} / s_{1}=r_{1}$ then $k=m s_{1}+n r_{1}, m, n$ non-negative integers. Since $\left(r_{1}, s_{1}\right)=1$, we can at least get the equality $k=m s_{1}+n r_{1}, m, n$ integers. If now $n$ is taken such that $0 \leqq n<s_{1}$, then $m$ must be positive since $k \geqq s_{1} r_{1}>n r_{1}$ : whence $\left(a_{j}\right)$ is true for $j=1$. Now $\left(b_{j}\right)$ follows from $\left(\mathrm{a}_{j-1}\right)$. In fact, since $t_{j} \equiv 0\left(s_{j}\right)$ we have $d_{j} \delta_{j}=t_{j} / t_{j-1} \cdot r_{j} / s_{j}=k / t_{j-1}, k$ an integer, and since $t_{j}>t_{j-1}$ and, by hypothesis, $r_{j} / s_{j}>d_{j-1} \delta_{j-1}$, we conclude that $k / t_{j-1}>d_{j-1} \delta_{j-1}$; thus by $\left(\mathrm{a}_{j-1}\right), t_{j} / t_{j-1} \cdot r_{j} / s_{j} \in \Gamma_{j-1}$.

Let $s_{j}^{\prime}=$ g.c.d. $\left(t_{j-1}, s_{j}\right)$; we have also $t_{j}=1$.c.m. $\left(t_{j-1}, s_{j}\right)$. Then $\dot{s}_{j}^{\prime} t_{j}=t_{j-1} s_{j}$. Now g.c.d. $\left(s_{j}^{2}, s_{j}^{\prime} t_{j}\right)=$ g.c.d. $\left(s_{j}^{2}, t_{j-1}, s_{j}\right)=s_{j} \cdot$ g.c.d. $\left(s_{j}, t_{j-1}\right)=s_{j} s_{j}^{\prime}$, whence 
g.c.d. $\left(s_{j} / s_{j-1}, t_{j} / t_{j-1}\right)=1 . s_{j} / s_{j}^{\prime}=t_{j} / t_{j-1}=d_{j}$. Let $t_{j} / s_{j}=s_{j}^{\prime \prime}$. Then $\left(r_{j} s_{j}^{\prime \prime}, d_{j}\right)=1$, whence there exists a non-negative integer $m<d_{j}$ such that $k-m r_{j} s_{j}^{\prime \prime} \equiv 0\left(d_{j}\right)$, whence

$$
\frac{k}{t_{j}}-\frac{m r_{j}}{s_{j}}=\frac{k}{t_{j}}-\frac{m r_{j} s_{j}^{\prime \prime}}{d_{j} s_{j}^{\prime} s_{j}^{\prime \prime}}=\frac{k^{\prime}}{s_{j}^{\prime} s_{j}^{\prime \prime}}=\frac{k^{\prime}}{t_{j-1}} \geqq \frac{r_{j}}{s_{j}}>d_{j-1} \frac{r_{j-1}}{s_{j-1}} .
$$

Statement $\left(\mathrm{a}_{j}\right)$ now follows by induction.

Let $K$ be an algebraically closed field of arbitrary characteristic, $\Sigma=K(x, y)$ a pure transcendental extension field of $K$. Let $v$ be a 0 -dimensional valuation of $\Sigma$ over $K$ with $v(x)>0, v(y)>0$. In the case that $v$ is of rank 1 , we may normalize the value group of $v$ by placing $v(x)=1$; the meaning of the phrase " $v(z)$ is a rational multiple of $v(x)$ " is then clear. In the case that $v$ is of rank 2, the phrase " $v(z)$ is a rational multiple of $v(x)$ " has the following precise meaning: there exist integers $r, s, s>0$, such that $v\left(z^{s}\right)=v\left(x^{r}\right)$.

Let $q_{1}, q_{2}, \cdots$ be the Jordan sequence of 0 -dimensional $v$-ideals in $K[x, y] ; \gamma_{i}=v\left(\mathfrak{q}_{i}\right)$. Let $\Delta_{0}$ be the group of values $\{m v(x)\}, m$ an integer. In case $v$ is of rank $1, v(x)$ is taken to be 1 , and $\Delta_{0}$ is simply the group of integers. Let $D_{i}$ be the additive abelian group generated by $v(x), \gamma_{1}, \gamma_{2}, \cdots, \gamma_{i}$. We have $\Delta_{0}=D_{0} \subseteq D_{1} \subseteq \cdots$. Let $\Delta_{j}$ be the $j$ th group $D_{i(j)}$ containing its predecessor properly; we also place $\Delta_{0}=D_{i(0)}$. Let $\Gamma_{0}$ be the set of values $\{m(v(x))\}$, $m$ a non-negative integer; $\Gamma_{j}$ the set of values $\left\{m+n_{1} \gamma_{i(1)}+\cdots+n_{j} \gamma_{i(j)}\right\}$, where $m, n_{i}$ are non-negative integers. We denote by $\delta_{1}, \delta_{2}, \cdots$ the integers $\gamma_{i(1)}, \gamma_{i(2)}, \cdots$.

THEOREM 6. Let $\Delta_{1} \subset \Delta_{2} \subset \cdots$ be the finite or infinite sequence of groups introduced above. There exists a corresponding sequence of polynomials, $f_{1}(x, y)$, $f_{2}(x, y), \cdots$, elements of $K[x, y]$, such that:

$\left(1_{j}\right) f_{j}(x, y)$ is monic in $y$, that is, the coefficient of the highest power of $y$ is 1 .

(2j) $v\left(f_{j}\right)=\delta_{j}, j \geqq 1$.

$\left(3_{j}\right)$ Degree in $y$ of $f_{j}$ is equal to $d_{j-1} \cdot$ degree in $y$ of $f_{j-1}, j>1$, where $d_{j-1}$ is an integer; degree in $y$ of $f_{1}$ is 1 .

$\left(4_{j}\right) d_{j-1} v\left(f_{j-1}\right) \in \Delta_{j-2} ; k v\left(f_{j-1}\right) \notin \Delta_{j-2}, 0<k<d_{j-1}, j>1$, where $k$ is an integer.

(5j) $v\left(f_{j}\right)>d_{j-1} v\left(f_{j-1}\right), j>1$.

Proof. If every $\gamma_{i}$ is an integral multiple of $v(x)$, we have $D_{0}=D_{1}=\cdots$, and there is nothing to prove; that is, the theorem is vacuously true. Suppose then that some $\gamma_{i}$, say $\gamma_{k}$, is not an integral multiple of $v(x)$. If $v(y)$ is not an integral multiple of $v(x)$ then $v(y)=\gamma_{i(1)}=\delta_{1}$, that is, any element $g(x, y)$ $\in K[x, y]$ of value less than $v(y)$ has an integral multiple of $v(x)$ as value. Thus Theorem 6 is satisfied, in this case, at least for $j=1\left(f_{1}=y\right)$. Suppose $v(y)=k_{1} v(x), k_{1}$ an integer. Since $v$ is 0 -dimensional and $K$ is algebraically closed, there exists an element $c_{1} \in K$ such that $v\left(y-c_{k} x^{k(1)}\right)>v(y)$; if $v\left(y-c_{1} x^{k(1)}\right)=k_{2} v(x), k_{2}$ an integer, then there exists an element $c_{2} \in K$ such that $v\left(y-c_{1} x^{k(1)}-c_{2} x^{k(2)}\right)>v\left(y-c_{1} x^{k(1)}\right)$. Suppose this process can be 
repeated $i$ times, so that we have integers $k_{1}, k_{2}, \cdots, k_{i+1}$ and elements $c_{1}, c_{2}, \cdots, c_{i} \in K$ such that $v\left(y-c_{1} x^{k(1)}-\cdots-c_{i} x^{k(i)}\right)=k_{i+1} v(x)$. Setting $\left.y_{1}=y-c_{1} x^{k(1)}-\cdots-c_{i} x^{k(i)}\right)$, we assert that $v\left(y_{1}\right)<\gamma_{k}$. In fact, let $g(x, y)$ be an element of $K[x, y]$ with $v(g)=\gamma_{k}$, and suppose $v\left(y_{1}\right)>v(g(x, y))$ (equality is not possible since $v(g)$ is not an integral multiple of $v(x))$. Then $g(x, y)=g\left(x, y_{1}+c_{1} x^{k(1)}+\cdots+c_{i} x^{k(i)}\right)=h\left(x, y_{1}\right)=h^{\prime}(x)+y_{1} h^{\prime \prime}\left(x, y_{1}\right)$, where $h^{\prime}(x) \in K[x], h^{\prime \prime}\left(x, y_{1}\right) \in K\left[x, y_{1}\right]=K[x, y]$. Thus $v\left(y_{1} h^{\prime \prime}\right)>v(g)$, whence $v(g)=v\left(h^{\prime}(x)\right)$, which is a contradiction, since $v\left(h^{\prime}\right)$ is an integer. Only a finite number of values less than $v(g)$ are assumed by elements of $K[x, y]$. Therefore, since $k_{1}<k_{2}<\cdots$, there exists an integer $m$ such that $y-c_{1} x^{k(1)}-\ldots$ $-c_{m} x^{k(m)}$ has a value not an integral multiple of $v(x)$. As above, $v\left(y-c_{1} x^{k(1)}\right.$ $\left.-\cdots-c_{m} x^{k(m)}\right)>v(g)$ is impossible, whence $v\left(y-c_{1} x^{k(1)}-\cdots-c_{m} x^{k(m)}\right)$ $=\gamma_{i(1)}=\delta_{1}$. Thus assertion 2 of the theorem is satisfied by $f_{1}(x, y)=y-c_{1} x^{k(1)}$ $-\cdots-c_{m} x^{k(m)}=y^{\prime}$. Also $f_{1}(x, y)$ is monic in $y$. Thus Theorem 6 is satisfied at least for $j=1,3$, parts 4,5 being so far vacuously true. Note that we may without loss of generality take $f_{1}(x, y)=y$, since $K[x, y]=K\left[x, y^{\prime}\right]$. and any monic polynomial $h\left(x, y^{\prime}\right)$ is transformed into a monic polynomial $h_{1}(x, y)$ $=h\left(x, y+c_{1} x^{k(1)}+\cdots+c_{m} x^{k(m)}\right)$ with $\operatorname{deg}_{y} h\left(x, y^{\prime}\right)=\operatorname{deg}_{y} h(x, y)$.

The proof now proceeds by induction on $j$. Suppose, then, that we have polynomials $f_{1}, f_{2}, \cdots, f_{j}$ satisfying conditions $1,2,3,4,5$. We have either to show that $D_{i(j)}=D_{i(j)+1}=\cdots$, or produce an $f_{j+1}(x, y) \in K[x, y]$ such that the five conditions are satisfied by $f_{1}, f_{2}, \cdots, f_{j}, f_{j+1}$.

Note that $v\left(f_{1}\right), v\left(f_{2}\right), \cdots, v\left(f_{j-1}\right)$ are rational multiples of $v(x)$. In fact, suppose $v\left(f_{1}\right), v\left(f_{2}\right), \cdots, v\left(f_{k}\right), k<j-1$, are rational multiples of $v(x)$; these values generate $\Delta_{k}$. Since $d_{k+1} v\left(f_{k+1}\right) \in \Delta_{k}$ by $4, v\left(f_{k+1}\right)$ is a rational multiple of $v(x)$. Thus $v\left(f_{1}\right), \cdots, v\left(f_{j-1}\right)$ are rational multiples of $v(x)$. Let $v\left(f_{k}\right)=\delta_{k}=r_{k} / s_{k} \cdot v(x), r_{k}, s_{k}$ positive integers, $\left(r_{k}, s_{k}\right)=1, k \leqq j-1$. Let $t_{0}=1$, $t_{k}=$ 1.c.m. $\left(s_{1}, \cdots, s_{k}\right)$. By Lemma 3 , in view of condition 5 , we have:

$\left(\mathrm{a}_{j-1}\right)$ If $k / t_{j-1} \cdot v(x) \geqq d_{j-1} v\left(f_{j-1}\right), k$ an integer, then $k / t_{j-1} \cdot v(x) \in \Gamma_{j-1}$.

$\left(\mathrm{b}_{j-1}\right) d_{j-1}=t_{j-1} / t_{j-2}$.

Note that if $v\left(f_{j}\right)$ is a rational multiple of $v(x)$, and $d_{j}$ is defined by $\left(4_{j+1}\right)$ that is, $d_{j}$ is the least positive integer such that $d_{j} v\left(f_{j}\right) \in \Delta_{j-1}$, then $\left(\mathrm{a}_{j}\right)$ and $\left(\mathrm{b}_{j}\right)$ hold.

We now prove:

(c) If $\operatorname{deg}_{y} g(x, y)<\operatorname{deg}_{y} f_{j}, g(x, y) \in K[x, y]$, then $g(x, y)$ is a sum of monomials in $x, f_{1}, \cdots, f_{j-1}$,

$$
g(x, y)=\sum c\left(\alpha_{0}, \cdots, \alpha_{j-1}\right) F\left(\alpha_{0}, \cdots, \alpha_{j-1}\right), c\left(\alpha_{0}, \cdots, \alpha_{j-1}\right) \in K,
$$

with $0 \leqq \alpha_{k}<d_{k}, k=1,2, \cdots, j-1$, in which no two monomials have equal values, and hence $v(g) \in \Delta_{j-1}$ where $F\left(\alpha_{0}, \cdots, \alpha_{j-1}\right)$ is the monomial $x^{\alpha(0)} f_{1}^{\alpha(1)} \cdots f_{j-1}^{\alpha(j-1)}$, a notation used throughout.

The proof is again by induction. For $j=1, \operatorname{deg}_{y} f_{1}=1$, whence $g(x, y)=g^{\prime}(x)$ $\in K[x]$, and the statement is trivial. For the general case, since $f_{j-1}(x, y)$ 
is monic in $y$ we may write

$$
g(x, y)=a_{0}(x, y)+a_{1}(x, y) f_{i-1}(x, y)+\cdots+a_{l}(x, y) f_{j-1}^{l}(x, y),
$$

$a_{i}(x, y) \in K[x, y]$, with $\operatorname{deg}_{y} a_{i}(x, y)<\operatorname{deg}_{y} f_{j-1}(x, y)$, and $l<d_{j-1}$ since $\operatorname{deg}_{y} g(x, y)<\operatorname{deg}_{y} f_{j}(x, y)=d_{j-1} \cdot \operatorname{deg}_{y} f_{j-1}(x, y)$. By induction, $v\left(a_{k}\right) \in \Delta_{j-2}$, $k=0,1, \cdots, l$, whence

$$
v\left(a_{k} f_{j-1}^{k}\right) \neq v\left(a_{k^{\prime}} f_{j-1}^{k^{\prime}}\right) \text { for } k \neq k^{\prime}
$$

by 4 , that is, since $v\left(f_{j-1}^{k-z^{\prime}}\right) \notin \Delta_{j-2}$. (c) now follows by induction. Note that if $v\left(f_{j}\right)$ is a rational of $v(x)$, and $d_{j}$ is defined by $\left(4_{j+1}\right)$, then $\left(c_{j+1}\right)$ holds when we write $d_{j} \operatorname{deg}_{v} f_{j}$ instead of $\operatorname{deg}_{v} f_{j+1}$.

If, now, $v\left(f_{j}\right)$ is not a rational multiple of $v(x)$ then $\Delta_{j}=D_{m}, m \geqq i_{j}$. In fact, if $g(x, y) \in K[x, y]$ then, since $f_{j}(x, y)$ is monic in $y$, we may write

$$
g(x, y)=a_{0}(x, y)+a_{1}(x, y) f_{j}(x, y)+\cdots+a_{l}(x, y) f_{j}^{l}(x, y),
$$

where $a_{i}(x, y) \in K[x, y]$, and $\operatorname{deg}_{y} a_{i}(x, y)<\operatorname{deg}_{y} f_{j}(x, y)$. By (c), $v\left(a_{k}\right) \in \Delta_{j-1}$ whence $v\left(a_{k} f_{j}^{k}\right) \neq v\left(a_{k^{\prime}}, f_{j}^{z^{\prime}}\right)$ for $k \neq k^{\prime}$ since $v\left(f_{j}^{k-z^{\prime}}\right)$ is not a rational multiple of $v(x)$, whereas $v\left(a_{k} / a_{k^{\prime}}\right)$ is. Thus $v(g(x, y))=v\left(a_{k}(x, y) f_{j}^{k}(x, y)\right)$, some $k, 0 \leqq k \leqq l$, whence $v(g(x, y)) \in \Delta_{j}$. In this case, as remarked before, there is nothing more to prove.

If $v\left(f_{j}\right)$ is a rational multiple of $v(x)$, we define $d_{j}$ by means of condition 4 . Since $d_{j} v\left(f_{j}\right)=k / t_{j-1}>d_{j-1} v\left(f_{j-1}\right)$, we have, by (a), $d_{j} v\left(f_{j}\right)=\alpha_{0}+\alpha_{1} \delta_{1}+\cdots$ $+\alpha_{j-1} \delta_{j-1}$, with $0 \leqq \alpha_{i}<d_{i}, i=1,2, \cdots, j-1$. Since $v$ is 0 -dimensional and $K$ is algebraically closed, there exists an element $c \in K$ such that $v\left(f_{j}^{d(j)}-c F\left(\alpha_{0}, \cdots, \alpha_{j-1}\right)\right)>\left(f_{j}^{d(j)}\right)$. Let $f_{j+1}^{(1)}(x, y)=f_{j}^{d(j)}-c F\left(\alpha_{0}, \cdots, \alpha_{j-1}\right)$. Now

$\left.\operatorname{deg}_{y} F\left(\alpha_{0}, \alpha_{1}, \cdots, \alpha_{j-1}\right)\right)<\operatorname{deg}_{y} F\left(0, d_{1}, d_{2}-1, d_{3}-1, \cdots, d_{j-1}-1\right)=\operatorname{deg}_{y} f_{j}$ whence $f_{j+1}^{(1)}$ is monic in $y$. If $v\left(f_{j+1}^{(1)}\right) \in \Delta_{j}$ then by $\left(a_{j}\right)$, one has

$$
v\left(f_{j+1}^{(1)}\right)=\alpha_{0}^{(2)}+\alpha_{1}^{(2)} \delta_{1}+\cdots+\alpha_{j}^{(2)} \delta_{j}, 0 \leqq \alpha_{i}^{(2)}<d_{i}, \quad i=1,2, \cdots, j .
$$

Then there exists an element $c_{2} \in K$ such that $v\left(f_{j+1}^{(1)}-c_{2} F\left(\alpha_{0}^{(2)}, \cdots, \alpha_{j}^{(2)}\right)\right)$ $>v\left(f_{j+1}^{(1)}\right)$. Let $f_{j+1}^{(2)}=f_{j+1}^{(1)}-c_{2} F\left(\alpha_{0}^{(2)}, \cdots, \alpha_{j}^{(2)}\right)$. Note, as above, that $f_{j+1}^{(2)}$ is monic in $y$. Repeating the above process, we obtain a sequence $f_{j+1}^{(1)}, f_{j+1}^{(2)}, \cdots$, which is finite if, for some integer $k, v\left(f_{j+1}^{(k)}\right) \notin \Delta_{j}$, and which is infinite otherwise.

In case the sequence is infinite, all groups $D_{m}, m \geqq i_{j}$, coincide with $\Delta_{j}$. In fact, if $g(x, y) \in K[x, y]$ and $v(g(x, y))=\gamma_{k}$ then there are only a finite number of values less than $v(g)$ assumed by elements in $K[x, y]$, whence for some $k, v\left(f_{j+1}^{(k)}\right)>v(g)$. Since $f_{j+1}^{(k)}$ is monic in $y, g(x, y)$ can be written:

$$
g(x, y)=a(x, y)+g^{\prime}(x, y) f_{j+1}^{(k)}(x, y),
$$


with $a(x, y), g^{\prime}(x, y) \in K[x, y]$ and $\operatorname{deg}_{y} a(x, y)<\operatorname{deg}_{y} f_{j+1}^{(k)}=\operatorname{deg}_{y} f_{j}^{d(j)}$, whence $v(g(x, y))=v(a(x, y)) \in \Delta_{j}$, by $\left(\mathrm{c}_{j+1}\right)$.

In case the sequence is not infinite, that is, in case for some integer $k$ we have $v\left(f_{j+1}^{(1)}\right), v\left(f_{j+1}^{(2)}\right), \cdots, v\left(f_{j+1}^{(k-1)}\right) \in \Delta_{j}$, while $v\left(f_{j+1}^{(k)}\right) \notin \Delta_{j}$, then either $v\left(f_{j+1}^{(k)}\right)$ is infinitely large with respect to $v(x)$ in which case $\Delta_{j}=D_{m}, m \geqq i_{j}$, or $f_{j+1}=f_{j+1}^{(k)}$ together with $f_{1}, \cdots, f_{j}$ satisfy $(1,2,3,4,5, j+1)$. In fact, in either case $1,3,4,5$, are clearly satisfied. It thus remains to prove in either case that if $v(g(x, y))<v\left(f_{j+1}\right), g(x, y) \in K[x, y]$, then $v(g) \in \Delta_{j}$. Since $f_{j+1}$ is monic in $y$ we may write $g(x, y)=g^{\prime}(x, y)+h(x, y) f_{j+1}(x, y)$, where $g^{\prime}(x, y), h(x, y) \in K[x, y]$ and $\operatorname{deg}_{y} g^{\prime}(x, y)<\operatorname{deg}_{y} f_{j+1}=\operatorname{deg}_{v} f_{j}^{d(j)}$, whence $v(g(x, y))=v\left(g^{\prime}(x, y)\right)$. The statement now follows from $\left(c_{j+1}\right)$. This completes the proof.

Corollary 1. The sequence $f_{1}, f_{2}, \ldots$ is infinite if and only if $v$ is nondiscrete, and of rational rank 1.

Proof. Let the sequence $f_{1}, f_{2}, \cdots$ be infinite. Since $\operatorname{deg}_{y} f_{i} \rightarrow \infty$ as $i \rightarrow \infty$, we have, by (c) of the above theorem, that $\lim \Delta_{j}$ is the value group. Since the value group contains an infinite ascending sequence of subgroups it must be non-discrete; since $v\left(f_{i}\right)$ is rationally dependent on $v(x)$, it is rational rank 1. The converse is trivial. (Actually, the corollary does not depend on any special properties of the $f_{j}$, but solely on the fact that the $f_{j}$ are in 1-1 correspondence with the $\Delta_{j}$; in fact, the value group is non-discrete, rational rank 1 if and only if the value group contains an infinite ascending sequence of subgroups).

COROLlary 2. If $v$ is of rank 2 , in which case the sequence $f_{1}, f_{2}, \cdots$ is finite, containing $j$ terms say, then, except for the valuation in which $v(y)$ is infinitely small with respect to $v(x)$, the possibilities (a) $\Delta \mathfrak{q}_{i} \neq 0$ and (b) $\Delta \mathfrak{q}_{i}=0$ correspond respectively to the possibilities $\left(\mathrm{a}^{\prime}\right) v\left(f_{j}\right)$ is a rational multiple of $v(x)$, and $\left(\mathrm{b}^{\prime}\right) v\left(f_{j}\right)$ is not a rational multiple of $v(x)$.

Proof. Also this corollary may be proved without recourse to any special properties of the $f_{i}$. In fact, in all cases of rank 2 valuations, if $\Delta \mathfrak{q}_{i} \neq 0$ then the $\gamma_{i}$ generate a group of the type of all integers (in fact, an isolated subgroup of the value group). Hence any two $\gamma$ 's are rationally dependent. In particular, then, if $v(y)$ is not infinitely small with respect to $v(x)$, then $x \notin \Delta q_{i}$ and the statement follows.

If $\Delta q_{i}=0$, the $\gamma_{i}$ generate the entire value group, which does not possess a rational base of one element. This completes the proof.

We wish to remark that if $v$ is of rank 1 then " $v\left(f_{j}\right)$ not a rational multiple of $v(x)$ " means $v\left(f_{j}\right)$ is an irrational number: if, however, $v$ is of rank 2, then " $v\left(f_{j}\right)$ not a rational multiple of $v(x)$ " can be distinguished into two cases. Namely, either $v\left(f_{j}\right)$ may be infinitely small with respect to $v(x)$; or, although $m v(x)>v\left(f_{j}\right)$ for some integer $m, v\left(x^{n}\right) \neq v\left(f_{j}^{n^{\prime}}\right)$ for any integers $n, n^{\prime}$ different 
from 0 . The former case can arise, however, only for $j=1$ and if $v(y)$ is infinitely small with respect to $v(x)$. (The case $v\left(f_{j}\right)$ infinitely large with respect to $v(x)$ cannot arise here, since $\left.i_{j} v(x)>\delta_{j}=v\left(f_{j}\right)\right)$.

Let $v$ be a rational rank 2 valuation of $\Sigma$ over $K$ with $v(x)>0, v(y)>0$ and let $f_{1}, f_{2}, \cdots, f_{j}$ be the polynomials for $v$ introduced in this theorem.

COROLlaRY 3. If $v_{1}$ is a rank 1 valuation of $\Sigma$ over $K$ (a subgroup of the additive group of real numbers being taken as a value group) and if $v_{1}(x)=v(x)$, $v_{1}\left(f_{i}\right)=v\left(f_{i}\right), i=1, \cdots, j$, then $v_{1}=v$.

Proof. We have shown that if $g(x, y) \in K[x, y]$ then $g(x, y)=a_{0}(x, y)$ $+a_{1}(x, y) f_{j}+\cdots+a_{l}(x, y) f_{j}^{l}$, where $a_{i}(x, y) \in K[x, y]$ and $\operatorname{deg}_{y} a_{i}(x, y)$ $<\operatorname{deg}_{y} f_{j}(x, y)$; moreover, that $v\left(a_{k} f_{j}^{k}\right) \neq v\left(a_{k}^{\prime} f_{j}^{k \prime}\right)$ for $k \neq k^{\prime}$. Hence by $(\mathrm{c}), g(x, y)$ is a sum of monomials in $x, f_{1}, \cdots, f_{j}$,

$$
g(x, y)=\sum c\left(\alpha_{0}, \cdots, \alpha_{j}\right) F\left(\alpha_{0}, \cdots, \alpha_{j}\right), c\left(\alpha_{0}, \cdots, \alpha_{j}\right) \in K,
$$

$\alpha_{i}, i=0,1, \cdots, j$, non-negative integers, with

$$
v\left(F\left(\alpha_{0}, \cdots, \alpha_{j}\right)\right) \neq v\left(F\left(\alpha_{0}^{\prime}, \cdots, \alpha_{j}^{\prime}\right)\right)
$$

unless $\alpha_{i}=\alpha_{i}^{\prime}, i=0,1, \cdots, j$. Hence the value of $g$ is the minimum of the values of these monomials. The corollary is an immediate consequence.

We insert here a table displaying the polynomials $f$ introduced in Theorem 6 for a given 0 -dimensional valuation. The valuation in which $v(y)$ is infinitely small with respect to $v(x)$ forms an exception, and is omitted.

\section{Rank 1}

Discrete $: f_{1}, f_{2}, \cdots, f_{j}, f_{j+1}^{(1)}, f_{j+1}^{(2)}, \cdots$ (infinite).

Non-discrete :

Rational Rank 1. $f_{1}, f_{2}, \cdots, f_{j}, f_{j+1}, \cdots$,

Rational Rank 2. $f_{1}, f_{2}, \cdots, f_{j}, f_{j+1}$.

\section{Rank 2}

$\Delta q_{i}=0 . \quad f_{1}, f_{2}, \cdots, f_{j}, f_{j+1}$.

$\Delta q_{i} \neq 0 . \quad f_{1}, f_{2}, \cdots, f_{j}, f_{j+1}^{(1)}, f_{j+1}^{(2)}, \cdots \quad$ (finite or infinite).

Let $v$ be a rational rank 2 valuation of $K(x, y)$ with $v(x)=1, v(y)>0$. Let $\Delta_{j+1}$ be the value group, and $f_{1}, f_{2}, \cdots, f_{j}, f_{j+1}, j \geqq 0$, the polynomials constructed in Theorem 6. $v\left(f_{j+1}\right)=\tau$ is an irrational number.

THEOREM 7. For any irrational number $\sigma>\tau=v\left(f_{i+1}\right)$ there exists a rational rank 2 valuation $v_{1}$ of $K(x, y)$ with $v_{1}(x)=1, v_{1}(y)>0$, and such that $v_{1}\left(f_{i}\right)=v\left(f_{i}\right)$, $i=1,2, \cdots, j, v_{1}\left(f_{j+1}\right)=\sigma$.

Proof. Since $f_{j+1}$ is monic in $y$, every polynomial $g(x, y) \in K[x, y]$ may be written

$$
g(x, y)=a_{0}(x, y)+a_{1}(x, y) f_{j+1}+\cdots+a_{k}(x, y) f_{j+1}^{k}
$$


with $a_{i}(x, y) \in K[x, y]$ and $\operatorname{deg}_{y} a_{i}<\operatorname{deg}_{y} f_{j+1}$. The expansion (1) for $g(x, y)$ is unique; in fact, let $g(x, y)=a_{0}^{\prime}(x, y)+a_{1}^{\prime}(x, y) f_{j+1}+\cdots+a_{k}^{\prime}(x, y) f_{j+1}^{k^{\prime}}$ with $a_{i}^{\prime}(x, y) \in K[x, y]$ and $\operatorname{deg}_{y} a_{i}<\operatorname{deg}_{y} f_{j+1}$. Since $a_{0}-a_{0}^{\prime} \equiv 0\left(f_{j+1}\right)$ and $\operatorname{deg}_{y} f_{j+1}>\operatorname{deg}_{y}\left(a_{0}-a_{0}^{\prime}\right)$ we have $a_{0}=a_{0}^{\prime}$. Similarly $k=k^{\prime}$ and $a_{i}=a_{i}^{\prime}$. By (c) of Theorem $6, v\left(a_{i}\right) \in \Delta_{j}$. We now place

$$
v_{1}(g)=\min \left(v\left(a_{i}\right)+i \sigma\right),
$$

where $i$ runs from 1 to $k$. Clearly $v_{1}\left(f_{i}\right)=v\left(f_{i}\right), i=1, \cdots, j, v_{1}\left(f_{j+1}\right)=\sigma$, $v_{1}(x)=1$ and $v_{1}(y)>0$. If $h(x, y)=b_{0}(x, y)+b_{1}(x, y) f_{j+1}+\cdots+b_{k^{\prime}}(x, y) f_{j+1}^{k^{\prime}}$ is the expansion (1) for a polynomial $h(x, y) \in K[x, y]$ then

$$
g+h=\left(a_{0}+b_{0}\right)+\left(a_{1}+b_{1}\right) f_{j+1}+\cdots+\left(a_{k^{\prime \prime}}+b_{k^{\prime \prime}}\right) f_{j+1}^{k^{\prime \prime}}
$$

is the expansion (1) for $g+h$, where $k^{\prime \prime}=\max \left(k, k^{\prime}\right)$ and $a_{i}=0$ for $i>k, b_{i}=0$ for $i>k^{\prime}$. Then

$$
\begin{aligned}
v_{1}(g+h) & =\min _{i}\left(v\left(a_{i}+b_{i}\right)+i \sigma\right) \geqq \min _{i}\left(\min \left(v\left(a_{i}\right), v\left(b_{i}\right)\right)+i \sigma\right) \\
& =\min _{i}\left(v\left(a_{i}\right)+i \sigma, v\left(b_{i}\right)+i \sigma\right)=\min \left(\min _{i}\left(v\left(a_{i}\right)+i \sigma\right)\right),
\end{aligned}
$$

$$
\left.\min _{i}\left(v\left(b_{i}\right)+i \sigma\right)\right)=\min \left(v_{1}(g), v_{1}(h)\right) \text {, }
$$

that is,

$$
v_{1}(g+h) \geqq \min \left(v_{1}(g), v_{1}(h)\right) .
$$

Moreover, since $v_{1}(1)=v_{1}(-1)=0$ we also have

$$
\left.v_{1}(g+h)=\min \left(v_{1}(g), v_{1}(h)\right) \text { if } v_{1}(g) \neq v_{1}(h)\right) .
$$

We wish now to prove

$$
v_{1}(g h)=v_{1}(g)+v_{1}(h) .
$$

Because of the uniqueness of (1) and because of $(2)$ and $\left(2^{\prime}\right)$ it is sufficient to prove (3) for $g=a_{i}(x, y) f_{j+1}^{\prime}, h=b_{i^{\prime}}(x, y) f_{j+1}^{t^{\prime}}$. Since the expansion (1) for $k(x, y) f_{y+1}^{m}, k(x, y) \in K[x, y]$, is simply obtained from the expansion for $k(x, y)$ by multiplication by $f_{j+1}^{m}$, we may assume, without loss of generality, that $i=i^{\prime}=0$, that is, $g=a_{i}(x, y), h=b_{i}(x, y)$. Since $\operatorname{deg}_{y} a_{i}<\operatorname{deg}_{y} f_{j+1}$ and $\operatorname{deg}_{y} b_{i}$ $<\operatorname{deg}_{y} f_{j+1}$, the expansion (1) for $g h$ is

$$
g h=a(x, y)+b(x, y) f_{j+1} .
$$

Since $v(g h)$ and $v(a)$ are elements of $\Delta_{j}$ while $v\left(b f_{j+1}\right) \in \Delta_{j}$, we have $v(g h)$ $=\min \left(v(a), v\left(b f_{j+1}\right)\right)$, whence $v(g h)=v(a)<v\left(b f_{j+1}\right)=v(b)+\tau<(b v)+\sigma$. Hence $v_{1}(g h)=v_{1}(a)=v(g h)=v(g)+v(h)=v_{1}(g)+v_{1}(h)$, which proves $(3)$.

If we now place

$$
v_{1}(g(x, y) / h(x, y))=v_{1}(g)-v_{1}(h)
$$


where $g(x, y), h(x, y) \in K[x, y], v_{1}$ becomes a valuation of $K(x, y)$. Since $v_{1}$ is of rational rank 2 , it is necessarily 0 -dimensional. This completes the proof.

Let $v$ be a 0 -dimensional valuation of $\Sigma$ over $K$ with $v(x)>0, v(y)>0$.

1. If $v$ is non-discrete, rational rank 1 , place $v(x)=1$, and let $f_{1}, f_{2}, \cdots$ be the infinite sequence of polynomials constructed in Theorem 6 .

2. If $v$ is rank 1 , discrete, or of rank 2 with $\Delta \mathfrak{q}_{i} \neq 0$ and $v(y)$ is not infinitely small with respect to $v(x)$, let $f_{1}, \cdots, f_{j} ; f_{j+1}^{(1)}, f_{j+1}^{(2)}, \cdots, j \geqq 0$, be the sequence of polynomials constructed in Theorem 6 . If $v$ is of rank 1 then the sequence $f_{j+1}^{(1)}, f_{j+1}^{(2)}, \cdots$ is infinite : this is also possible for $v$ of rank 2 , but the case $v\left(f_{j+1}^{(k)}\right)$ infinitely great with respect to $v(x)$ can arise. To conserve notation, we place $f_{j+1}^{(\boldsymbol{k})}=f_{j+1}^{(\boldsymbol{k}+1)}=\cdots$, in the latter case.

3. If $v$ is rational rank 2 or of rank 2 with $\Delta \mathfrak{q}_{i}=0$, let $f_{1}, f_{2}, \cdots, f_{k}, f_{k+1}$, $k \geqq 0$, be the sequence of polynomials constructed in Theorem 6 .

In all three cases we write $\pi(v(g))=r / s, g \in \Sigma$, if $s v(g)=r v(x), r, s$ integers, $s \neq 0$.

THEOREM 8.1. For any integer $j>0$ and any irrational number $\tau>v\left(f_{j}^{d(j)}\right)$ there exists a rational rank 2 valuation $v_{1}$ of $\Sigma$ over $K$ with $v_{1}(x)=1, v_{1}(y)>0$ such that $v_{1}\left(f_{i}\right)=v\left(f_{i}\right), i=1, \cdots, j$, and $v_{1}\left(f_{j+1}\right)=\tau$.

ThEOREM 8.2. For any integer $k>0$ and any irrational number $\tau>\pi\left(v\left(f_{j}^{d(j)}\right)\right)$, there exists a rational rank 2 valuation $v_{1}$ of $\Sigma$ over $K$ with $v_{1}(x)=1, v_{1}(y)>0$, $v_{1}\left(f_{i}\right)=\pi\left(v\left(f_{i}\right)\right), i=1, \cdots, j$, and $v_{1}\left(f_{j+1}^{(k)}\right)=\tau .\left(\right.$ For $j=0$, place $\left.f_{j}^{d(j)}=y.\right)$

THEOREM 8.3. For any $j, 0 \leqq j \leqq k$, and any irrational number $\tau>\pi\left(v\left(f_{j}^{d(j)}\right)\right)$, there exists a rational rank 2 valuation $v_{1}$ of $\Sigma$ over $K$ with $v_{1}(x)=1, v_{1}(y)>0$ such that $v_{1}\left(f_{i}\right)=\pi\left(v\left(f_{i}\right)\right), i=1, \cdots, j, v_{1}\left(f_{j+1}\right)=\tau$. (For $j=0$, place $f_{j}^{d(j)}=y$; for $k=0$, the theorem is trivial, where $\tau$ is any positive irrational number.)

Proof. By Theorem 6 there exists a monomial $c F\left(\gamma_{0}, \cdots, \gamma_{j-1}\right), c \in K$, $0 \leqq \gamma_{i}<d_{i}, i=1, \cdots, j-1$, such that $v\left(F\left(0, \cdots, 0, d_{j}\right)-c F\left(\gamma_{0}, \cdots, \gamma_{j-1}\right)\right)$ $>v\left(F\left(0, \cdots, 0, d_{j}\right)\right)$. Let $\bar{f}_{j+1}^{\prime}=F\left(0, \cdots, 0, d_{j}\right)-c F\left(\gamma_{0}, \cdots, \gamma_{j-1}\right)$. If $v$ is non-discrete, rational rank 1 , or rank 1 , discrete, then $v\left(\bar{f}_{j+1}\right)$ is certainly a rational multiple of $v(x)$; if $v$ is rank 2 with $\Delta q_{i} \neq 0$ (as in Theorem 8.2) then $v\left(\bar{f}_{j+1}\right)$ is possibly infinitely large with respect to $v(x)$ (in which case $\bar{f}_{j+1}=f_{j+1}^{(1)}$ $\left.=f_{j+1}^{(2)}=\cdots\right)$ or $v\left(\bar{f}_{j+1}\right)$ is a rational multiple of $v(x)$; in Theorem $8.3, v\left(\bar{f}_{j+1}\right)$ will be a rational multiple of $v(x)$, unless $j=k$, and $\bar{f}_{j+1}=f_{k+1}$. In case $v\left(\bar{f}_{j+1}\right)$ is a rational multiple of $v(x)$, it is sufficient, by Theorem 7 , to prove Theorem 8 for an irrational number $\tau$ such that

$$
\pi v\left(F\left(0, \cdots, 0, d_{j}\right)\right)<\tau<\pi v\left(F\left(0, \cdots, 0, d_{j}\right)-c F\left(\gamma_{0}, \cdots, \gamma_{j-1}\right)\right),
$$

since any number greater than $\pi v\left(f_{j}^{d(j)}\right)$ is also greater than some irrational number satisfying this inequality. Since $\bar{f}_{j+1}$ is monic in $y$, any polynomial $g(x, y) \in K[x, y]$ can be written as 


$$
g(x, y)=a_{0}(x, y)+a_{1}(x, y) \bar{f}_{j+1}+\cdots+a_{k}(x, y) \bar{f}_{j+1}^{k},
$$

where $a_{i}(x, y) \in K[x, y]$ and $\operatorname{deg}_{y} a_{i}(x, y)<\operatorname{deg}_{y} \bar{f}_{j+1}$. Just as in Theorem 7 , one proves that the expansion (1) is unique. Placing

$$
v_{1}(g)=\min _{i}\left(\pi\left(v\left(a_{i}\right)\right)+i \tau\right),
$$

we can prove exactly as in Theorem 7 that

$$
v_{1}(g+h) \geqq \min \left(v_{1}(g), v_{1}(h)\right)
$$

and

$$
v_{1}(g+h)=\min \left(v_{1}(g), v_{1}(h)\right) \text { if } v_{1}(g) \neq v_{1}(h)
$$

where $g, h \in K[x, y]$. Also as before, in proving

$$
v_{1}(g h)=v_{1}(g)+v_{1}(h)
$$

we may assume without loss of generality that

$$
\operatorname{deg}_{y} g(x, y)<\operatorname{deg}_{y} \bar{f}_{j+1}=\operatorname{deg}_{y} f_{j}^{d(j)} \text { and } \operatorname{deg}_{y} h(x, y)<\operatorname{deg}_{y} \bar{f}_{j+1}=\operatorname{deg}_{y} f_{j}^{d(\eta)} \text {. }
$$

By (c) of Theorem 6, $g(x, y), h(x, y)$ are sums of monomials $c F\left(\alpha_{0}, \cdots, \alpha_{j}\right)$, $c \in K, 0 \leqq \alpha_{i}<d_{i}, i=1, \cdots, j$, of distinct value. Hence by the uniqueness of (1), and because of (2) and (2'), we may further assume without loss in generality that

$$
g(x, y)=F\left(\alpha_{0}, \cdots, \alpha_{j}\right), \quad 0 \leqq \alpha_{0}, 0 \leqq \alpha_{i}<d_{i}, i=1, \cdots, j
$$

and

$$
h(x, y)=F\left(\beta_{0}, \cdots, \beta_{i}\right), \quad 0 \leqq \beta_{0}, 0 \leqq \beta_{i}<d_{i}, i=1, \cdots, j,
$$

or, more generally, that

$g(x, y)=g^{\prime}(x, y) f_{i}^{\alpha(j)}$, where $g^{\prime}(x, y) \in K[x, y], \operatorname{deg}_{y} g^{\prime}<\operatorname{deg}_{y} f_{i}$, and $\alpha_{i}<d_{i}$, and

$h(x, y)=h^{\prime}(x, y) f_{j}^{\beta(j)}$, where $h^{\prime}(x, y) \in K[x, y] \operatorname{deg}_{y} h^{\prime}<\operatorname{deg}_{y} f_{j}$, and $\beta_{i}<d_{j}$.

Consider first the case $\alpha_{j}=0$. If $\beta_{j}<d_{j}-1$ then $\operatorname{deg}_{y} g h=\operatorname{deg}_{\nu} g^{\prime} h^{\prime} f_{j}^{\beta(j)}$ $<\operatorname{deg}_{v} f_{j}^{2} f_{j}^{d(j)-2}=\operatorname{deg}_{y} f_{j}^{d(j)}$, whence $v_{1}(g h)=\pi v(g h)=\pi v(g)+\pi v(h)=v_{1}(g)+v_{1}(h)$. If $\beta_{j}=d_{j}-1$, then

$$
\begin{aligned}
g h & =g^{\prime} h^{\prime} f_{j}^{d(j)-1}=\left(a(x, y)+b(x, y) f_{j}\right) f_{j}^{d(j)-1} \\
& =a(x, y) f_{j}^{d(j)-1}+b(x, y)\left(\bar{f}_{j+1}+c F\left(\gamma_{0}, \cdots, \gamma_{j-1}\right)\right)
\end{aligned}
$$

where $a, b \in K[x, y]$ and $\operatorname{deg}_{y} a<\operatorname{deg}_{\nu} f_{j}, \operatorname{deg}_{y} b<\operatorname{deg}_{y} f_{j}$. Also, $v\left(g^{\prime} h^{\prime}\right)=\min (v(a)$, 
$\left.v\left(b f_{j}\right)\right)=v(a)$, that is,

$$
v(a)<v\left(b f_{j}\right)
$$

since $v\left(g^{\prime} h^{\prime}\right), v(a) \in \Delta_{j-1}$ while $v\left(b f_{j}\right) \notin \Delta_{j-1}$. Because of the limited degree in $y$ of $a(x, y)$ and $b(x, y)$,

$$
\begin{aligned}
v_{1}\left(a f_{j}^{d(j)-1}\right) & =\pi\left(v\left(a f_{j}^{d(j)-1}\right)\right), \\
v_{1}\left(b f_{j}^{d(j)}\right) & =\min \left(\pi(v(b))+\tau, \quad \pi\left(v\left(b \cdot F\left(\gamma_{0}, \cdots, \gamma_{j-1}\right)\right)\right)\right) \\
& =\pi\left(v\left(b \cdot F\left(\gamma_{0}, \cdots, \gamma_{j-1}\right)\right)\right)=\pi\left(v\left(b \cdot f_{j}^{d(j)}\right)\right)>\pi\left(v\left(a f_{j}^{d(j)-1}\right)\right) \\
& =v_{1}\left(a f_{j}^{d(j)-1}\right)
\end{aligned}
$$

whence

$$
v_{1}(g h)=v_{1}\left(a f_{j}^{d(j)-1}\right)=\pi\left(v\left(a f_{j}^{d(j)-1}\right)\right)=\pi(v(g h))=\pi v(g)+\pi v(h)=v_{1}(g)+v_{1}(h) .
$$

Consider now the case $\alpha_{j}>0$, the general case. Here we can write, for reasons given in the case $\alpha_{j}=0$,

$$
g h=g^{\prime} h^{\prime} f_{j}^{\alpha(j)+\beta(j)}=\left(a(x, y)+b(x, y) f_{j}\right) f_{j}^{\alpha(j)+\beta(j)},
$$

with the same conditions on $a(x, y)$ and $b(x, y)$, and again deduce that $v(a)$ $<v\left(b f_{j}\right)$. We assert that

$$
v_{1}\left(a f_{j}^{\alpha(j)+\beta(j)}\right)=\pi v\left(a f_{j}^{\alpha(j)+\beta(j)}\right)
$$

and that

$$
v_{1}\left(b f_{j}^{\alpha(j)+\beta(j)+1}\right)=\pi v\left(b f_{j}^{\alpha(j)+\beta(j)+1}\right),
$$

whence will follow,

$$
\begin{aligned}
v_{1}(g h) & \left.=\min \left(v_{1}\left(a f_{j}^{\alpha(j)+\beta(j)}\right)\right), v_{1}\left(b f_{j}^{\alpha(j)+\beta(j)+1}\right)\right)=\pi\left(v\left(a f_{j}^{\alpha(j)+\beta(j)}\right)\right) \\
& =\pi(v(g h))=\pi(v(g))+\pi(v(h))=v_{1}(g)+v_{1}(h) .
\end{aligned}
$$

Since $\alpha_{j}<d_{j}$ and $\beta_{j}<d_{j}$ we have $\alpha_{j}+\beta_{j}+1 \leqq 2 d_{j}-1$. If $\alpha_{j}+\beta_{j}+1<d_{j}$ then $\operatorname{deg}_{y} b f_{j}^{\alpha(j)+\beta(j)+1}<\operatorname{deg}_{y} f_{j}(j)$, whence

$$
v_{1}\left(b f_{j}^{\alpha(j)+\beta(j)+1}\right)=\pi\left(v\left(b f_{j}^{\alpha(j)+\beta(j)+1}\right)\right) .
$$

If $\alpha_{j}+\beta_{j}+1 \geqq d_{j}$ we can write

$$
b f_{j}^{\alpha(j)+\beta(j)+1}=b f_{j}^{\alpha(j)+\beta(j)+1-d(j)}\left(\bar{f}_{j+1}+c F\left(\gamma_{0}, \cdots, \gamma_{j-1}\right)\right) .
$$

Since $\operatorname{deg}_{y} b f_{j}^{\alpha(j)+\beta(j)+1-d(j)}<\operatorname{deg}_{y} f_{j}^{d(j)}$,

$$
v_{1}\left(b f_{j}^{\alpha(j)+\beta(j)+1-d(j)} \bar{f}_{j+1}\right)=\pi v\left(b f_{j}^{\alpha(j)+\beta(j)+1-d(j)}\right)+\tau,
$$

and since $\alpha_{j}+\beta_{j}+1-d_{j}<d_{j}$, we have by the case $\alpha_{j}=0$, 


$$
\begin{aligned}
v_{1}\left(b f _ { j } ^ { \alpha ( j ) + \beta ( j ) + 1 - d ( j ) } \cdot F \left(\gamma_{0}, \cdots,\right.\right. & \left.\left.\gamma_{j-1}\right)\right) \\
& =v_{1}\left(b f_{j}^{\alpha(j)+\beta(j)+1-d(j)}\right)+v_{1}\left(F\left(\gamma_{0}, \cdots, \gamma_{j-1}\right)\right) \\
& =\pi\left(v\left(b f_{j}^{\alpha(j)+\beta(j)+1-d(j)}\right)+\pi\left(v\left(f_{j}^{d(j)}\right)\right),\right.
\end{aligned}
$$

whence

$$
v_{1}\left(b f_{j}^{\alpha(j)+\beta(j)+1)}=v_{1}\left(b f_{j}^{\alpha(j)+\beta(j)+1-d(j)} \cdot F\left(\gamma_{0}, \cdots, \gamma_{j-1}\right)\right)=\pi\left(v\left(b f_{j}^{\alpha(j)+\beta(j)+1}\right)\right)\right. \text {. }
$$

Similarly $v_{1}\left(a f_{j}^{\alpha(j)+\beta(j)}\right)=\pi\left(v\left(a f_{j}^{\alpha(j)+\beta(j)}\right)\right)$.

Thus (3) is proved. Placing $v_{1}(g / h)=v_{1}(g)-v_{1}(h), g / h$ any element of $\Sigma$, $g, h \in K[x, y]$, we obtain that $v_{1}$ is a valuation of $\Sigma$ over $K$. Since $v_{1}$ is of rational rank 2 , it is 0 -dimensional. That $v_{1}(x)=1, v_{1}\left(f_{i}\right)=\pi\left(v\left(f_{i}\right)\right), i=1, \cdots, j$, is immediate from the definition of $v_{1} . f_{1}$ is monic in $y$ of degree 1 , whence $f_{1}-y \equiv 0(x)$, since $v\left(f_{1}-y\right)>0$. Hence $v_{1}(y)>0$, since $v_{1}\left(f_{1}\right)>0$ and $v_{1}(x)>0$.

It remains to prove the conditions on $v_{1}\left(f_{j+1}\right), v_{1}\left(f_{j+1}^{(k)}\right)$, respectively. In the case $v\left(\bar{f}_{j+1}\right)$ is not a rational multiple of $v(x), \bar{f}_{j+1}=f_{k+1}$ or $\bar{f}_{j+1}=f_{j+1}^{(k)}$ and the condition has been verified. In the other cases we assume $\tau<\pi v\left(\bar{f}_{j+1}\right)$. Recalling the construction of $f_{j+1}^{(k)}$, note that $f_{j+1}^{(1)}=\bar{f}_{j+1}$. Thus for $k=1$, the required statement holds. Now unless $f_{j+1}^{(k)}=f_{j+1}^{(1)}$, we have $\operatorname{deg}_{y}\left(f_{j+1}^{(k)}-f_{j+1}^{(1)}\right)<\operatorname{deg}_{y} f_{j}^{d(j)}$, whence $v_{1}\left(f_{j+1}^{(k)}-f_{j+1}^{(1)}\right)=\pi\left(v\left(f_{j+1}^{(k)}-f_{j+1}^{(1)}\right)\right)$. Hence,

$$
v_{1}\left(f_{j+1}^{(k)}\right)=v_{1}\left(f_{j+1}^{(1)}+\left(f_{j+1}^{(k)}-f_{j+1}^{(1)}\right)\right)=\min \left(\tau, \pi\left(v\left(f_{j+1}^{(k)}-f_{j+1}^{(1)}\right)\right)\right)=\tau,
$$

since $\tau<\pi v\left(f_{j+1}^{(1)}\right)=\pi\left(v\left(f_{j+1}^{(k)}-f_{j+1}^{(1)}\right)\right)$. The statement for $v_{1}\left(f_{j+1}\right)$ follows similarly if we consider $f_{j+1}$ constructed from $f_{j}$ as in Theorem 6 . Note also, however, that since $f_{j+1}$ and $\bar{f}_{j+1}$ are both monic in $y$ of the same degree it is true that $v\left(f_{j+1}-\bar{f}_{j+1}\right) \in \Delta_{j}$ by (c) of Theorem 6. Hence $v\left(f_{j+1}-\bar{f}_{j+1}\right) \geqq v\left(\bar{f}_{j+1}\right)$, since $v\left(f_{j+1}\right) \notin \Delta_{j}$. The statement for $f_{j+1}$ now follows exactly as above for $f_{j+1}^{(k)}$. This completes the proof.

Remark. For $j=0$, the restriction $\tau>v\left(f_{j}^{d(j)}\right)=v(y)$ is superfluous with $\tau>0$ only required. In fact, for $j=0$, and the restriction on $\tau$ lifted, Theorems 8.2 and 8.3 become: For any monic polynomial $f_{1} \in K[x, y]$ of degree 1 in $y$, and any irrational number $\tau>0$, there exists a rational rank 2 valuation $v_{1}$ such that $v_{1}(x)=1, v_{1}(y)>0$, and $v_{1}\left(f_{1}\right)=\tau$. This is clear directly, without reference to the theorem.

Let $v$ be a 0 -dimensional valuation of $\Sigma$ over $K$ with $v(x)>0, v(y)>0$ and let $\mathfrak{q}_{1}, \mathfrak{q}_{2}, \cdots$ be the Jordan sequence of 0 -dimensional $v$-ideals in $K[x, y]$ for $v$.

THEOREM 9. For any integer $m>0$ there exists a valuation $v_{1}$ of $\Sigma$ over $K$ of rational rank 2 with $v_{1}(x)=1, v_{1}(y)>0$ such that $q_{1}, q_{2}, \cdots, q_{m}$ are the first $m$ valuation-ideals for $v_{1}$ in $K[x, y]$.

Proof. Consider first the case that $v$ is non-discrete and rational rank 1 ; let $v(x)=1$, and let $f_{1}, f_{2}, \cdots$ be the (infinite) sequence of polynomials intro- 
duced in Theorem 6. We assert that if $\mathfrak{q}$ is a $v$-ideal with $v(\mathfrak{q})<v\left(f_{j+1}\right)$ then $\mathfrak{q}$ has a basis consisting of $f_{j+1}$ and monomials $F\left(\alpha_{0}, \cdots, \alpha_{j}\right), 0 \leqq \alpha_{0}, 0 \leqq \alpha_{i}<d_{i}$, $i=1, \cdots, j$. In fact, if $g(x, y) \in q$ then, since $f_{j+1}$ is monic in $y$,

$$
g(x, y)=g^{\prime}(x, y)+g^{\prime \prime}(x, y) f_{j+1}(x, y),
$$

where $g^{\prime}(x, y), g^{\prime \prime}(x, y) \in K[x, y]$ and $\operatorname{deg}_{y} g^{\prime}(x, y)<\operatorname{deg}_{y} f_{j+1}(x, y)$. Since $f_{j+1} \in \mathfrak{q}$, also $g^{\prime}=g-g^{\prime \prime} f_{j+1} \in q$, whence the statement follows by (c) of Theorem 6 . Since $q_{m}$ is preceded by (contained in) only a finite number of $v$-ideals for $v$, there exists an integer $j=j(m)$ such that $v\left(\mathfrak{q}_{m}\right)<v\left(f_{j+1}\right)$. Consider the set of monomials $F\left(\alpha_{0}, \cdots, \alpha_{j}\right), 0 \leqq \alpha_{0}, 0 \leqq \alpha_{i}<d_{i}, i=1, \cdots, j=j(m)$, and order them according to their value: $F\left(\alpha_{0}, \cdots, \alpha_{j}\right)$ precedes $F\left(\alpha_{0}^{\prime}, \cdots, \alpha_{j}^{\prime}\right)$ if $v\left(F\left(\alpha_{0}, \cdots, \alpha_{j}\right)\right)<v\left(F\left(\alpha_{0}^{\prime}, \cdots, \alpha_{j}^{\prime}\right)\right)$. Since every $F\left(\alpha_{0}, \cdots, \alpha_{j}\right)$ has only a finite number of predecessors, the ordered set $\left\{F\left(\alpha_{0}, \cdots, \alpha_{j}\right)\right\}$ is a simple sequence:

$$
u_{1}, u_{2}, \cdots \text {. }
$$

For some $k, v\left(u_{k}\right)<v\left(f_{j+1}\right)<v\left(u_{k+1}\right)$, where clearly $k \geqq m$; consider also the sequence:

$$
u_{1}, u_{2}, \cdots, u_{k}, f_{j+1}, u_{k+1}, u_{k+2}, \cdots .
$$

Every $v$-ideal $\mathfrak{q}$ with $v(\mathfrak{q})<v\left(f_{j+1}\right)$ has for value the value of some $u_{i}, i \leqq k$, and conversely, whence $v\left(q_{i}\right)=v\left(u_{i}\right), i=1, \cdots, k$. Since $q_{i}, i=1, \cdots, k$, has a basis consisting of elements in (2), the $\mathfrak{q}_{i}$ may be described in the following manner: $q_{i}$ has a basis consisting of $u_{i}$ and the elements of (2) following $u_{i}, i=1, \cdots, k$. Since $v\left(f_{f}^{d()}\right)=v\left(u_{i}\right)$, some $i$, and $v\left(f_{j}^{d(j)}\right)<v\left(f_{j+1}\right)$, we have $i \leqq k$, whence $v\left(u_{k}\right) \geqq v\left(f_{j}^{d(j)}\right)$. Thus if $\tau$ is an irrational number with $v\left(u_{k}\right)<\tau<v\left(u_{k+1}\right)$ then there exists, by Theorem 8 , a rational rank 2 valuation of $\Sigma$ over $K$ with $v_{1}(x)=1, v_{1}(y)>0$ such that $v_{1}\left(f_{i}\right)=v\left(f_{i}\right), i=1, \cdots, j$, and $v_{1}\left(f_{i+1}\right)=\tau$. Since $v_{1}\left(u_{i}\right)=v\left(u_{i}\right)$, every $i$, and $v_{1}\left(u_{k}\right)<v_{1}\left(f_{j+1}\right)<v_{1}\left(u_{k+1}\right)$, the set of monomials $\left\{F\left(\alpha_{0}, \cdots, \alpha_{j}\right)\right\}, 0 \leqq \alpha_{0}, 0 \leqq \alpha_{i}<d_{i}, i=1, \cdots, j$, together with $f_{j+1}$ ordered according to their value $v_{1}$, again yields the sequence (2). The $v_{1}$-ideals of $v_{1}$-value less than $v_{1}\left(f_{j+1}\right)$ can be described again as above: the $i$ th $v_{1}$-ideal has for basis $u_{i}$ and the elements of (2) following $u_{i}, i=1, \cdots, k$. Since $m \leqq k, \mathfrak{q}_{1}, \cdots, \mathfrak{q}_{m}$ are the first $m v_{1}$-ideals.

Next, consider the case that $v$ is discrete, rank 1 or of rank 2 with $\Delta q_{i} \neq 0$ (leaving aside the case $v(y)$ is infinitely small with respect to $v(x)$, for which case the theorem is readily verified). In this case, the sequence $f_{1}, f_{2}, \ldots$ of Theorem 6 is finite, containing $j$ terms, say. If $v$ is of rank 1 then the sequence $f_{j+1}^{(1)}, f_{j+1}^{(2)}, \ldots$ is infinite: this is also possible for $v$ of rank 2 , but the case $v\left(f_{j+1}^{(k)}\right)$ infinitely great with respect to $v(x)$ can arise. To conserve notation, we place $f_{j+1}^{(k)}=f_{j+1}^{(k+1)}=\cdots$, in the latter case. Now, since $q_{m}$ is contained in only a finite number of $v$-ideals, there exists an integer $l$ such that $v\left(\mathfrak{q}_{m}\right)<v\left(f_{j+1}^{(l)}\right)$. The ideal $\mathfrak{q}_{i}, i=1, \cdots, m$, then has a basis consisting of $f_{j+1}^{(l)}$ 
and monomials $F\left(\alpha_{0}, \cdots, \alpha_{j}\right), 0 \leqq \alpha_{0}, 0 \leqq \alpha_{i}<d_{i}, i=1, \cdots, j$. We order the set $\left\{F\left(\alpha_{0}, \cdots, \alpha_{j}\right)\right\}$ by means of $v$, again obtaining the sequence (1); and if $v\left(f_{j+1}^{(l)}\right)=v\left(u_{k}\right)$ we also introduce the sequence:

$$
u_{1}, u_{2}, \cdots, u_{k}, f_{j+1}^{(l)}, u_{k+1}, \cdots .
$$

Introducing a rational rank 2 valuation $v_{1}$ of $\Sigma$ over $K$ with $v_{1}(x)=1, v_{1}(y)>0$ such that $v_{1}\left(f_{i}\right)=\pi\left(v\left(f_{i}\right)\right), i=1, \cdots, j$, where $\pi\left(v\left(f_{i}\right)\right)=r / s$ if $v\left(f_{i}^{s}\right)=v\left(x^{r}\right)$, and $v_{1}\left(f_{j+1}^{(l)}\right)=\tau$, where $\tau$ is an irrational number satisfying $\pi\left(u_{k}\right)<\tau<\pi\left(u_{k+1}\right)$, we can conclude as above that $q_{1}, \cdots, q_{m}$ are the first $m v_{1}$-ideals.

Finally, we have the case that $v$ is of rank 2 with $\Delta \mathfrak{q}_{i}=0$. The sequence $f_{1}, f_{2}, \cdots$ is finite, containing $j+1$ terms, say. Here every $v$-ideal has a basis consisting of monomials $F\left(\alpha_{0}, \cdots, \alpha_{j}, \alpha_{j+1}\right), 0 \leqq \alpha_{0}, 0 \leqq \alpha_{j+1}, 0 \leqq \alpha_{i}<d_{i}$, $i=1,2, \cdots, j$, and two distinct such monomials have distinct value. These may be ordered according to their value $v$, and again we obtain the sequence (1). We introduce a rational rank 2 valuation $v_{1}$ of $\Sigma$ over $K$ with $v_{1}(x)=1$, $v_{1}(y)>0, v_{1}\left(f_{i}\right)=v\left(f_{i}\right), i=1, \cdots, j, v_{1}\left(f_{j+1}\right)=\tau>v\left(f_{j}^{d(j)}\right), \tau$ irrational. The valuation $v_{1}$ also orders the set $\left\{F\left(\alpha_{0}, \cdots, \alpha_{j}, \alpha_{j+1}\right)\right\}, 0 \leqq \alpha_{0}, 0 \leqq \alpha_{j+1}$, $0 \leqq \alpha_{i}<d_{i}, i=1, \cdots, j$, yielding the simple sequence

$$
w_{0}, w_{1}, \cdots
$$

corresponding to (1). We wish to determine the extent to which $\left(1^{\prime}\right)$ coincides with (1); or rather, how large an initial segment of (1) may be made to coincide with an initial segment of $\left(1^{\prime}\right)$ by a proper choice of $\tau$. To this end, consider two terms of (1):

$$
F\left(\alpha_{0}, \cdots, \alpha_{j}, \alpha_{j+1}\right), \quad F\left(0, \beta \cdots, \beta_{j}, \beta_{j+1}\right)
$$

with $v\left(F\left(\alpha_{0}, \cdots, \alpha_{j}, \alpha_{j+1}\right)\right)<v\left(F\left(\beta_{0}, \cdots, \beta_{j}, \beta_{j+1}\right)\right)$. If $\alpha_{j+1}=\beta_{j+1}$ then these two terms will have the same order in $\left(1^{\prime}\right)$ as in (1), no matter what $\tau$, since $v_{1}\left(F\left(\alpha_{0}, \cdots, \alpha_{j}\right)\right)=v\left(F\left(\alpha_{0}, \cdots, \alpha_{j}\right)\right), v_{1}\left(F\left(\beta_{0}, \cdots, \beta_{j}\right)\right)=v\left(F\left(\beta_{0}, \cdots, \beta_{j}\right)\right)$. Thus we need consider only the cases $\alpha_{j+1}<\beta_{j+1}$ and $\alpha_{j+1}>\beta_{j+1}$. In case $\alpha_{j+1}>\beta_{j+1}$ we shall use the letters $\gamma, \delta$ instead of $\alpha, \beta$; that is, consider two terms of (1):

$$
F\left(\gamma_{0 j} \cdots, \gamma_{j}, \gamma_{j+1}\right), \quad F\left(\delta_{0}, \cdots, \delta_{j}, \delta_{j+1}\right)
$$

with $v\left(F\left(\gamma_{0}, \cdots, \gamma_{j}, \gamma_{j+1}\right)\right)<v\left(F\left(\delta_{0}, \cdots, \delta_{j}, \delta_{j+1}\right)\right)$, where $\gamma_{j+1}>\delta_{j+1}$ and $\alpha_{j+1}<\beta_{j+1}$ is now understood in (3). The two terms of (3) will have the same order in ( $\left.1^{\prime}\right)$ if and only if $\tau$ is such that

$$
\pi\left(v\left(F\left(\alpha_{0}, \cdots, \alpha_{j}\right)\right)\right)+\alpha_{i+1} \tau<\pi\left(v\left(F\left(\beta_{0}, \cdots, \beta_{j}\right)\right)\right)+\beta_{j+1} \tau,
$$

that is, if and only if,

$$
\tau>\frac{\pi\left(v\left(F\left(\alpha_{0}, \cdots, \alpha_{j}\right)\right)\right)-\pi\left(v\left(F\left(\beta_{0}, \cdots, \beta_{j}\right)\right)\right)}{\beta_{j+1}-\alpha_{j+1}} .
$$


For the appropriate terms, condition (4) includes the condition $\tau>d_{j} v\left(f_{j}\right)$, since $d_{j} v\left(f_{j}\right)$ is the value of some $u_{i}$ with $v\left(u_{i}\right)<v\left(f_{j+1}\right)$. Similarly, the two terms of $\left(3^{\prime}\right)$ will have the same order in $\left(1^{\prime}\right)$ if and only if

$$
\tau<\frac{\pi\left(v\left(F\left(\delta_{0}, \cdots, \delta_{j}\right)\right)\right)-\pi\left(v\left(F\left(\gamma_{0}, \cdots, \gamma_{j}\right)\right)\right)}{\gamma_{j+1}-\delta_{j+1}} .
$$

Now

$$
\begin{aligned}
\left(\gamma_{j+1}-\delta_{j+1}\right) v\left(F\left(\alpha_{0}, \cdots, \alpha_{j}\right) / F\right. & \left.\left(\beta_{0}, \cdots, \beta_{j}\right)\right) \\
& <\left(\beta_{j+1}-\alpha_{j+1}\right)\left(\gamma_{j+1}-\delta_{j+1}\right) v\left(f_{j+1}\right) \\
& <\left(\beta_{j+1}-\alpha_{j+1}\right) v\left(F\left(\delta_{0}, \cdots, \delta_{j}\right) / F\left(\gamma_{0}, \cdots, \gamma_{j}\right)\right)
\end{aligned}
$$

by (3) and ( $\left.3^{\prime}\right)$, whence

$$
\begin{aligned}
\frac{\pi\left(v\left(F\left(\alpha_{0}, \cdots, \alpha_{j}\right)\right)\right)-\pi\left(v\left(F\left(\beta_{0}, \cdots, \beta_{j}\right)\right)\right)}{\beta_{i+1}-\alpha_{i+1}} & <\frac{\pi\left(v\left(F\left(\delta_{0}, \cdots, \delta_{j}\right)\right)\right)-\pi\left(v\left(F\left(\gamma_{0}, \cdots, \gamma_{j}\right)\right)\right)}{\gamma_{i+1}-\delta_{j+1}} .
\end{aligned}
$$

Thus $\tau$ can be taken such that (4) and (4') be satisfied. Similarly, any finite number of conditions (4) and (4') are satisfied by some irrational number $\tau$. Thus for any initial segment of (1) there exists a valuation $v_{1}$ such that the elements of this segment retain the same relative order in $\left(1^{\prime}\right)$; that is, for any integer $n>0$ there exists a valuation $v_{1}=v_{1}(n)$ such that if $u_{i}=w_{k(i)}$ then $k_{i}<k_{i+1}$ provided $i<n$. For a given $i, k_{i}$ is a function of $v_{1}: k_{i}=k_{i}\left(v_{1}\right)$. Let $k^{(i)}=\min \left\{k_{i}\left(v_{1}\right)\right\}$ over all $v_{1}$. We assert that $k^{(i)} \rightarrow \infty$ as $i \rightarrow \infty$. In fact, consider the monomials $\left\{F\left(\alpha_{0}, \cdots, \alpha_{j}\right)\right\}, 0 \leqq \alpha_{0}, 0 \leqq \alpha_{i}<d_{i}, i=1, \cdots, j$, and order them according to their $v$-values, which are also their $v_{1}$-values, obtaining the sequence:

$$
z_{1}, z_{2}, \cdots \text {. }
$$

Clearly the sequence (1) may also be written:

$$
z_{l(1)} f_{j+1}^{m(1)}, z_{l(2)} f_{j+1}^{m(2)}, \cdots \text {. }
$$

We have

$$
k^{(i)} \geqq \max \left(l_{i}, m_{i}\right),
$$

since $v_{1}\left(w_{k(i)}\right)=v_{1}\left(u_{i}\right)=v_{1}\left(z_{l(i)} f_{j+1}^{m(i)}\right) \geqq \max \left(v_{1}\left(z_{l(i)}\right), v_{1}\left(f_{j+1}^{m(i)}\right)\right)$. Since not both $l_{i}, m_{i}$ are bounded, $k^{(i)} \rightarrow \infty$ as $i \rightarrow \infty$. Hence for any integer $N>0$ there exists an integer $n=n(N)$ such that if $u_{i}=w_{k}$, where $k=k_{i}\left(v_{1}\right)$, then $k_{i}\left(v_{1}\right)>N$ for any $v_{1}$, provided $i>n$. Thus if the elements $u_{1}, u_{2}, \cdots, u_{n}$ retain their order in $\left(1^{\prime}\right)$ then the initial segment $u_{1}, \cdots, u_{N}$ of (1) coincides with the initial segment $w_{1}, \cdots, w_{N}$ of $\left(1^{\prime}\right)$. 
Let $s$ be an integer such that $v\left(\mathfrak{q}_{m}\right)<v\left(f_{j+1}^{s}\right)$. Then every ideal $\mathfrak{q}_{1}, \cdots, \mathfrak{q}_{m}$ has a basis consisting of $f_{j+1}^{s}$ and monomials $F\left(\alpha_{0}, \alpha_{1}, \cdots, \alpha_{j}, \alpha_{j+1}\right), 0 \leqq \alpha_{0}$, $0 \leqq \alpha_{i}<d_{i}, i=1, \cdots, j, \alpha_{j+1}<s$. In fact, if $g(x, y) \in q_{i}, i \leqq m$, then, since $f_{j+1}$ and $f_{j+1}^{s}$ are monic in $y$ one can write:

$$
g(x, y)=a_{0}(x, y)+a_{1}(x, y) f_{j+1}+\cdots+a_{s-1}(x, y) f_{j+1}^{s-1}+a_{s}(x, y) f_{j+1}^{s}
$$

where $a_{i}(x, y) \in K[x, y], i=0,1, \cdots, s$, and $\operatorname{deg}_{y} a_{i}<\operatorname{deg}_{y} f_{j+1}, i=0,1, \cdots$, $s-1$. The statement now follows from (c) of Theorem 6 . We can therefore describe the ideals $q_{1}, \cdots, q_{m}$ in terms of the sequence (1) in the following manner: $q_{k}$ has a basis consisting of the elements $u_{i}$ with $i \geqq k$. If, now, $v_{1}$ is such that the initial segment of (1) preceding $f_{j+1}^{s}$ coincides with an initial segment of $\left(1^{\prime}\right)$, one concludes, in a manner employed above, that $q_{1}, \cdots, q_{m}$ are the first $m v$-ideals of $v_{1}$. This completes the proof.

The significance of Theorem 9 is that it allows us in the study of 0 -dimensional $v$-ideals of 0 -dimensional valuations to restrict ourselves to rational rank 2 valuations, at least if this study is confined to the ring $K[x, y]$.

COROLlARY 1. In the case $v$ is non-discrete, rational rank 1 , or rank 1 and discrete, or of rank 2 with $\Delta q_{i} \neq 0$, the $v_{1}$-ideals of value less than $\tau$ are the first $v$-ideals for $v$. In the case $v$ is of rank 2 with $\Delta q_{i}=0$, the $v_{1}$-ideals of value less than st are the first $v$-ideals for $v$ (where $s$ is as in the theorem).

COROLlaRY 2. If the $v_{1}$-ideals of $v_{1}$-value less than st are the first $v$-ideals for $v$ and if $\tau>1$ then:

(a) in the case that $v$ is non-discrete, rational rank $1, s \leqq d_{j+1}$,

(b) in the case that $v$ is rank 1 and discrete or of rank 2 with $\Delta q_{i} \neq 0, s=1$.

Proof. Suppose $s \geqq d_{j+1}$. Then the $v_{1}$-ideals of $v_{1}$-value less than $v_{1}\left(f_{j+1}^{d(j+1)}\right)$ are the first $v$-ideals for $v$. We order the elements $F\left(\alpha_{0}, \cdots, \alpha_{j+1}\right), 0 \leqq \alpha_{0}$, $0 \leqq \alpha_{i}<d_{i}, i=1, \cdots, j+1$, according to their $v$ - and $v_{1}$-value, obtaining the sequences

$$
\begin{aligned}
& u_{1}, u_{2}, \cdots, \\
& w_{1}, w_{2}, \cdots .
\end{aligned}
$$

If $v_{1}\left(w_{k}\right)<v_{1}\left(f_{j+1}^{d(j+1)}\right)<v_{1}\left(w_{k+1}\right)$, then as in the theorem it follows that $u_{i}=w_{i}$, $i=1, \cdots, k$. Let $\mathfrak{q}_{1}, \mathfrak{q}_{2}, \cdots ; \mathfrak{q}_{1}^{*}, \mathfrak{q}_{2}^{*}, \cdots$ be the Jordan sequences of 0 dimensional valuation ideals for $v$ and $v_{1}$ respectively. We know that $v\left(f_{j+1}^{d(j+1)}\right)=v\left(u_{m}\right)$, for some $u_{m}$, and since $q_{k}=q_{k}^{*}$, we have $m \geqq k$. If $m=k$ then $\mathfrak{q}_{k+1} \neq \mathfrak{q}_{k+1}^{*}$ since $f_{j+1}^{d(j+1)} \in \mathfrak{q}_{k+1}^{*}$ but $f_{j+1}^{d(j+1)} \in \mathfrak{q}_{k+1}$, whence $s>d_{j+1}$ is impossible. If $m>k$ then $\mathfrak{q}_{k+1}=\mathfrak{q}_{k+1}^{*}$ but $\mathfrak{q}_{k+2} \neq \mathfrak{q}_{k_{+2}}^{*}$, since $u_{k+1} \in \mathfrak{q}_{k+2}^{*}$ but $u_{k+1} \notin \mathfrak{q}_{k+2}$. Now if $s>d_{j+1}$ then $s \tau>d_{j+1} \tau+1$, whence $v_{1}\left(\mathfrak{q}_{k+2}\right)<v_{1}\left(f_{j+1}^{s}\right)$. But then $\mathfrak{q}_{k+2}=\mathfrak{q}_{k+2}^{*}$, a contradiction. This completes the proof of (a). Part (b) follows in the same way if we place $d_{j+1}=1$ (and write $f_{j+1}^{(l)}$ for $f_{j+1}$ ). This completes the proof.

6. Characterization of simple $v$-ideals. Let $v$ be a 0 -dimensional valuation 
of $\Sigma$ over $K$ with $v(x)>0, v(y)>0$. If we further assume that $v(y) \geqq v(x)$ then the groups $D_{0}, D_{1}, \cdots$ introduced in Theorem 6 no longer depend on the element $x$, but solely on the ring $K[x, y]$; in fact, $v(y) \geqq v(x)>0$ implies that $v\left(\mathfrak{q}_{1}\right)=v(x)$, and we may write $v\left(\mathfrak{q}_{1}\right)$ instead of $v(x)$ in Theorem 6. If $v$ does not belong to the set of non-discrete rational rank 1 valuations, then for some integer $j$ we have $\Delta_{j}=D_{m}, m \geqq i_{j}$. This integer $j$ depends only on $v$ and on the ring $K[x, y]$. We shall call $j$ the type of $v$ with respect to the ring $K[x, y]$.

THEOREM 10. Let $v$ be a rational rank 2 valuation of $\Sigma$ over $K$ with $0<v(x)<v(y)$ and of type $j$ with respect to $K[x, y], j>1$, and let $f_{1}, f_{2}, \cdots, f_{j}$ be the polynomials introduced in Theorem 6 . Then the v-ideals for $v$ of value equal to or less than $d_{j-1} v\left(f_{j-1}\right)$ are also the first $v$-ideals for a rational rank 2 valuation $v_{1}$ of $\Sigma$ over $K$ with $0<v_{1}(x)<v_{1}(y)$ and of type $j-1$ with respect to $K[x, y]$. The polynomials $f_{1}, \cdots, f_{j-1}$ satisfy Theorem 6 for the valuation $v_{1}$.

Proof. If $\mathfrak{q}$ is a $v$-ideal with $v(\mathfrak{q}) \leqq d_{j-1} v\left(f_{j-1}\right)$ then $\mathfrak{q}$ has a basis consisting of $f_{j-1}^{d(j-1)}$ together with monomials $\left\{F\left(\alpha_{0}, \cdots, \alpha_{j-1}\right)\right\}, 0 \leqq \alpha_{0}, 0 \leqq \alpha_{i}<d_{i}$, $i=1, \cdots, j-1$. As in Theorem 9, we order the set $\left\{F\left(\alpha_{0}, \cdots, \alpha_{j-1}\right)\right\}$, $0 \leqq \alpha_{0}, 0 \leqq \alpha_{i}<d_{i}, i=1, \cdots, j-1$, by means of $v$, obtaining the simple sequence:

$$
u_{1}, u_{2}, \cdots \text {. }
$$

If $v\left(f_{j-1}^{d(j-1)}\right)=v\left(u_{k}\right)$ then any $v$-ideal $\mathfrak{q}_{i}$ with $i \leqq k$ can be described in the following manner: $q_{i}$ has a basis consisting of $f_{j-1}^{d(j-1)}$ together with the elements $u_{i^{\prime}}$ of (1) with $i^{\prime} \geqq i$. Normalizing $v$ so as to have $v(x)=1$, we introduce for every irrational $\tau>d_{j-2} v\left(f_{j-2}\right)$ (if $j=2$, for every irrational $\tau>1$ ) a rational rank 2 valuation $v_{1}$ of $\Sigma$ over $K$ with $v_{1}(x)=1, v_{1}(y)>0, v_{1}\left(f_{i}\right)=v\left(f_{i}\right)$, $i=1, \cdots, j-2$, and $v_{1}\left(f_{j-1}\right)=\tau$. The valuation $v_{1}$ also orders the set $\left\{F\left(\alpha_{0}, \cdots, \alpha_{j-1}\right)\right\}, 0 \leqq \alpha_{0}, 0 \leqq \alpha_{i}<d_{i}, i=1, \cdots, j-1$, yielding the simple sequence :

$$
w_{1}, w_{2}, \cdots
$$

corresponding to (1). Now $\left|v\left(u_{i}\right)-v_{1}\left(u_{i}\right)\right|<d_{j-1}\left|\tau-v\left(f_{j-1}\right)\right|$, since $\alpha_{j-1}<d_{j-1}$ in any term $u_{i}$, whence, if

$$
d_{j-1}\left|\tau-v\left(f_{j-1}\right)\right|<\min _{i=1, \cdots, k}\left|v\left(u_{i}\right)-v\left(u_{i+1}\right)\right|
$$

then the initial segment $u_{1}, \cdots, u_{k}$ of (1) coincides with an initial segment of $\left(1^{\prime}\right)$. (We may note that if $v_{1}\left(u_{i}\right)>v\left(u_{i}\right)$ for one $u_{i}$ then $v_{1}\left(u_{i}\right) \geqq v\left(u_{i}\right)$ for every $i$.) Let $V_{1}$ denote the set of valuations $\left\{v_{1}\right\}$ for which (2) holds. For every valuation $v_{1} \in V_{1}$ such that $\tau>v\left(f_{j-1}\right)$, the ideals $q_{1}, \cdots, \mathfrak{q}_{k}$ are the first $k v_{1}$-ideals; in fact $d_{j-1} v_{1}\left(f_{j-1}\right)>d_{j-1} v\left(f_{j-1}\right)=v\left(u_{k}\right)=v_{1}\left(u_{k}\right)=v_{1}\left(w_{k}\right)\left(v\left(u_{k}\right)=v_{1}\left(u_{k}\right)\right.$, since by condition 4 of Theorem $6, u_{k}$ does not involve $\left.f_{j-1}\right)$, whence we can draw the required conclusion in a manner several times employed in Theo- 
rem 9 . The polynomials $f_{1}, f_{2}, \cdots, f_{j-1}$ clearly satisfy Theorem $\sigma_{j-1}$. Since $v_{1}\left(f_{i-1}\right)$ is irrational, the sequence $f_{1}, f_{2}, \cdots$ for $v_{1}$ terminates, as is shown in Theorem 6 , with $f_{j-1}$; that is, $v_{1}$ is of type $j-1$ with respect to $K[x, y]$. It remains to prove $v_{1}(y)>v_{1}(x)$. If $f_{1}=y$, this is clear. If $f_{1} \neq y$, then $v\left(f_{1}-y\right)$ is an integer, and since $v\left(f_{1}\right)$ is not an integer, $v\left(f_{1}-y\right)=v(y) \geqq 2$. Hence also $v_{1}\left(f_{1}-y\right) \geqq 2$, since $v_{1}\left(f_{1}-y\right)=v\left(f_{1}-y\right)$. Since $v_{1}\left(f_{1}\right)>1, v_{1}(y)>1=v_{1}(x)$ follows. This completes the proof.

CoROLLARY 1. The ideals $\mathfrak{q}_{1}, \cdots, \mathfrak{q}_{k}$ are the first $k v_{1}$-ideals for any valuation $v_{1} \in V_{1}$.

Proof. For $\tau>v\left(f_{j-1}\right)$ the proof has been given above. The proof there depends on the fact that, since $d_{j-1} v\left(f_{j-1}\right)>v\left(u_{k}\right)=v_{1}\left(u_{k}\right)=v_{1}\left(w_{k}\right)$, the $k$ th $v_{1}$-ideal has a basis consisting of $f_{j-1}^{d(f-1)}$ together with the monomials $w_{i}$ with $i \geqq k$. If $\tau<v\left(f_{j-1}\right)$ then $d_{j-1} v\left(f_{j-1}\right)<v\left(u_{k}\right)=v_{1}\left(u_{k}\right)=v_{1}\left(w_{k}\right)$. However, if $d_{j-1} v\left(f_{j-1}\right)$ $>v_{1}\left(w_{k-1}\right)$ then the $k$ th $v_{1}$-ideal can still be described as before: it has a basis consisting of $f_{j-1}^{d(j-1)}$ together with the monomials $w_{i}$ with $i \geqq k$, whence, as before, $\mathfrak{q}_{k}$ would be the $k$ th $v_{1}$-ideal. And, in fact, $v\left(u_{k-1}\right) \geqq v_{1}\left(u_{k-1}\right)$, whence

$$
d_{j-1} v\left(f_{j-1}\right)-d_{j-1} v_{1}\left(f_{j-1}\right)<v\left(u_{k}\right)-v\left(u_{k-1}\right) \leqq v\left(u_{k}\right)-v_{1}\left(u_{k-1}\right),
$$

by (2), and $d_{j-1} v_{1}\left(f_{j-1}\right)>v_{1}\left(u_{k-1}\right)$. This completes the proof.

CoRollary 2. The $v$-ideai $\mathfrak{q}_{k}$ with $v\left(\mathfrak{q}_{k}\right)=d_{j-1} v\left(f_{j-1}\right)$ is a simple $v$-ideal.

Proof. Since $v\left(f_{j-1}\right)>d_{j-2} v\left(f_{j-2}\right), \tau=v_{1}\left(f_{j-1}\right), v_{1} \in V_{1}$, may be taken greater than or less than $v\left(f_{j-1}\right)$. If $\tau>v\left(f_{j-1}\right)$ then $d_{j-1} v_{1}\left(f_{j-1}\right)>d_{j-1} v\left(f_{j-1}\right)=v\left(u_{k}\right)$ $=v_{1}\left(u_{k}\right)=v_{1}\left(q_{k}\right)$, whence $v_{1}\left(q_{k}\right)$ is rational, while if $\tau<v\left(f_{j-1}\right)$ then $d_{j-1} v_{1}\left(f_{j-1}\right)$ $<v_{1}\left(u_{k}\right)$, whence $v_{1}\left(q_{k}\right)=d_{j-1} v_{1}\left(f_{j-1}\right)$, that is, $\dot{v}_{1}\left(q_{k}\right)$ is irrational. Suppose now that $q_{k}$ is not simple: $q_{k}=q_{k(1)} q_{k(2)} \cdots q_{k(m)}, m>1$. Since $u_{k(l)}, l=1,2, \cdots, m$, belong to the initial segment $u_{1}, u_{2}, \cdots, u_{k-1}$, we have

$$
v_{1}\left(\mathfrak{q}_{k(l)}\right)=v_{1}\left(u_{k(l)}\right), \quad l=1, \cdots, m,
$$

and $v_{1}\left(\mathfrak{q}_{k}\right)=v_{1}\left(u_{k(1)}\right)+\cdots+v_{1}\left(u_{k(m)}\right)$. Let $v_{1}\left(u_{k(l)}\right)=r_{k(l)}+s_{k(l)} v_{1}\left(f_{j-1}\right)$, where $r_{k(l)}$ is a non-negative rational number, and $s_{k(l)}$ is a non-negative integer, $l=1, \cdots, m$. The numbers $r_{k(l)}, s_{k(l)}$ are independent of the valuation $v_{1}: s_{k(l)}$ is the exponent of $f_{j-1}$ in $u_{k(l)}$, while $r_{k(l)}=v_{1}\left(u_{k(l)} f_{j-1} \exp s_{k(l)}\right)$ is the same for any $v_{1} \in V_{1}$, since $u_{k(l)} / f_{j-1} \exp s_{k(l)}$ is a monomial in $x, f_{1}, f_{2}, \cdots, f_{j-2}$. If now $v_{1} \in V_{1}$ is taken such that $v_{1}\left(q_{k}\right)$ is irrational $\left(=d_{j-1} v_{1}\left(f_{j-1}\right)\right)$, we have that $r_{k(1)}+\cdots+r_{k(m)}=0$, whence $r_{k(1)}=\cdots=r_{k(l)}=0$. But then $v_{1}\left(u_{k(l)}\right)$ $=s_{k(m)} v_{1}\left(f_{j-1}\right)$ for every $v_{1} \in V_{1}, l=1, \cdots, m$, whence $v_{1}\left(q_{k}\right)=\left(s_{k(1)}+\cdots\right.$ $\left.+s_{k(m)}\right) v_{1}\left(f_{j-1}\right)$, that is, $v_{1}\left(q_{k}\right)$ is irrational for every $v_{1} \in V_{1}$. This is a contradiction.

CoROllaRy 3. All the $v$-ideals $\mathfrak{q}_{i}$ with $d_{j-1} v\left(f_{j-1}\right) \leqq v\left(\mathfrak{q}_{i}\right) \leqq v\left(f_{j}\right)$ are simple.

Proof. For $v\left(\mathfrak{q}_{i}\right)=d_{j-1} v\left(f_{j-1}\right)$, this is Corollary 2. If $v\left(\mathfrak{q}_{i}\right)=v\left(f_{j}\right)$, then $\mathfrak{q}_{i}$ is 
certainly simple, since $v\left(\mathfrak{q}_{i}\right)$ is irrational, while $v\left(\mathfrak{q}_{1}\right), v\left(\mathfrak{q}_{2}\right), \cdots, v\left(\mathfrak{q}_{i-1}\right)$ are rational. Suppose $v\left(\mathfrak{q}_{i}\right)=u_{i}<v\left(f_{j}\right), i>k$, where $v\left(u_{k}\right)=d_{j-1} v\left(f_{j-1}\right)$. Since $v\left(u_{i-1}\right) \geqq d_{j-1} v\left(f_{j-1}\right)$, there exists by Theorem 8.3 for any irrational number $\tau, v\left(u_{i-1}\right)<\tau<v\left(u_{i}\right)$, a rational rank 2 valuation $v_{1}$ of $\Sigma$ over $K$ with $v_{1}(x)=1, v_{1}(y)>0$ such that $v_{1}\left(f_{i}\right)=v\left(f_{i}\right), i=1, \cdots, j-1$ and $v_{1}\left(f_{j}\right)=\tau$. The first $i v_{1}$-ideals (in $K[x, y]$ ) may clearly be described in the following manner : the $l$ th $v_{1}$-ideal, $l \leqq i$, has a basis consisting of $f_{j}$ and the monomials $u_{k}$ with $k \geqq l$. Hence, $\mathfrak{q}_{1}, \mathfrak{q}_{2}, \cdots, \mathfrak{q}_{i}$ are the first $i v_{1}$-ideals. Moreover, since $v_{1}\left(f_{j}\right)$ $<v_{1}\left(u_{i}\right)$, we have that $v\left(\mathfrak{q}_{i}\right)=v_{1}\left(f_{j}\right)=\tau$. This case, however, has already been considered. This completes the proof.

CoRollary 4. Every $v$-ideal $\mathfrak{q}_{i}$ with $d_{j-1} v\left(f_{j-1}\right)<v\left(\mathfrak{q}_{i}\right) \leqq v\left(f_{j}\right)$ belongs to a rational rank 2 valuation $v_{1}$ of $\Sigma$ over $K$ with $v_{1}(x)=1, v_{1}(y)>0$, with $f_{1}, \cdots, f_{i}$ as associated polynomials, and with $\mathfrak{q}_{1}, \cdots, \mathfrak{q}_{i}$ as first v-ideals, such that $v_{1}\left(\mathfrak{q}_{i}\right)=v_{1}\left(f_{j}\right)=\tau^{\prime}$.

Proof. This has just been proved in Corollary 3 .

Let $v$ be a rational rank 2 valuation of $\Sigma$ over $K$ with $v(x)=1$ and $v(y)>0$, and let $f_{1}, f_{2}, \cdots, f_{n}$ be the sequence of polynomials for $v$ introduced in Theorem 6 . We have $v\left(f_{i}\right)=r_{i} / s_{i}, r_{i}, s_{i}$ positive integers, $\left(r_{i}, s_{i}\right)=1, i=1$, $\cdots, n-1$, and $v\left(f_{n}\right)=\tau$ is irrational. Every $v$-ideal has a basis consisting of monomials in $x, f_{1}, \cdots, f_{n}$, thus every ideal has a value $u / t+v \tau, u, v$ nonnegative integers, where $t=t_{n-1}=1 . \operatorname{c.m} .\left(s_{1}, \cdots, s_{n-1}\right)$.

Lemma. If $v\left(F\left(\alpha_{0}, \cdots, \alpha_{k}\right)\right)>v\left(F\left(\beta_{0}, \cdots, \beta_{k}\right)\right)+v\left(f_{k+1}\right)-1,0 \leqq \alpha_{i}, 0 \leqq \beta_{i}$ $i=0,1, \cdots, k$, then there exist non-negative integers $\alpha_{i}^{\prime}, \beta_{i}^{\prime}$ such that $\alpha_{i}^{\prime} \geqq \beta_{i}^{\prime}$, $v\left(F\left(\alpha_{0}^{\prime}, \cdots, \alpha_{k}^{\prime}\right)\right)=v\left(F\left(\alpha_{0}, \cdots, \alpha_{k}\right)\right), v\left(F\left(\beta_{0}^{\prime}, \cdots, \beta_{k}^{\prime \prime}\right)\right)=v\left(F\left(\beta_{0}, \cdots, \beta_{k}\right)\right)^{\prime}$.

Proof. The proof is by induction on $k$, being trivial for $k=0$. (That is, $\alpha_{0}>\beta_{0}-1$, whence $\alpha_{0} \geqq \beta_{0}$.)

First, if $\alpha_{k}<\beta_{k}$ then $v\left(F\left(\alpha_{0}, \cdots, \alpha_{k-1}\right)\right)>v\left(F\left(\beta_{0}, \cdots, \beta_{k-1}\right)\right)+v\left(f_{k}\right)-1$, so that by induction we may assume $\alpha_{i} \geqq \beta_{i}, i=0,1, \cdots, k-1$. Factoring out $F\left(\beta_{0}, \cdots, \beta_{k-1}\right)$, we may assume $\beta_{0}=\beta_{1}=\cdots=\beta_{k-1}=0$. Assuming $\beta_{0}=\beta_{1}=\cdots=\beta_{k-1}=0$, let $\alpha_{k}$ be as large as possible, that is, let $\alpha_{k}=\max \alpha_{k}^{\prime}$ over the set of monomials $F\left(\alpha_{0}^{\prime}, \cdots, \alpha_{k}^{\prime}\right)$ of value $=v\left(F\left(\alpha_{0}, \cdots, \alpha_{k}\right)\right)$, where $0 \leqq \alpha_{i}^{\prime}, i=0,1, \cdots, k$; that is, we may without loss of generality suppose that $\alpha_{k}^{\prime} \leqq \alpha_{k}$ if $v\left(F\left(\alpha_{0}^{\prime}, \cdots, \alpha_{k}^{\prime}\right)\right)=v\left(F\left(\alpha_{0}, \cdots, \alpha_{k}\right)\right)$, where $0 \leqq \alpha_{i}^{\prime}$, $i=0,1, \cdots, k$. Then $\alpha_{k} \geqq \beta_{k}$. In fact, supposing $\alpha_{k}<\beta_{k}$, then $v\left(F\left(\alpha_{0}, \cdots, \alpha_{k-1}\right)\right)$ $>v\left(f_{k}\right)+v\left(f_{k+1}\right)-1>d_{k} v\left(f_{k}\right)+v\left(f_{k}\right)-1$. Now $d_{k} v\left(f_{k}\right)=v\left(F\left(\gamma_{0}, \cdots, \gamma_{k-1}\right)\right)$. By induction we may assume that $\alpha_{i} \geqq \gamma_{i}, i=0,1, \cdots, k-1$. But then

$$
v\left(F\left(\alpha_{0}, \cdots, \alpha_{k-1}, \alpha_{k}\right)\right)=v\left(F\left(\alpha_{0}-\gamma_{0}, \cdots, \alpha_{k-1}-\gamma_{k-1}, \alpha_{k}+d_{k}\right)\right)
$$

which is in contradiction with the maximality of $\alpha_{k}$.

Second, if $\alpha_{k} \geqq \beta_{k}$, we may assume, factoring out $f_{k} \exp \beta_{k}$, that $\beta_{k}=0$. Assuming $\beta_{k}=0$, let $\alpha_{k}$ be as small as possible, that is, let $\alpha_{k}=\min \alpha_{k}^{\prime}$ over the 
set of monomials $F\left(\alpha_{0}^{\prime}, \cdots, \alpha_{k}^{\prime}\right)$ of value $=v\left(F\left(\alpha_{0}, \cdots, \alpha_{k}\right)\right)$, where $0 \leqq \alpha_{i}^{\prime}$, $i=0,1, \cdots, k$. Then certainly $\alpha_{k}<d_{k}$, whence $v\left(F\left(\alpha_{0}, \cdots, \alpha_{k-1}\right)\right)$ $>v\left(F\left(\beta_{0}, \cdots, \beta_{k-1}\right)\right)+v\left(f_{k}\right)-1$, since $v\left(f_{k+1}\right)>v\left(f_{k}^{d(k)}\right)>v\left(f_{k}^{\alpha(k)}\right)$. The proof is thus complete by induction.

The essential facts of the following theorem have already been proved for $n=1$. Principally on the grounds of simplicity, therefore, we assume $n>1$.

TheOREM 11. Let $v$ be as in the lemma, and let $n>1$.

1. If $v\left(\mathfrak{q}_{i}\right)=u / t+v \tau$, then $\mathfrak{q}_{i}=\mathfrak{q}_{j} \mathfrak{q}_{k}$, where $v\left(\mathfrak{q}_{j}\right)=u / t, v\left(\mathfrak{q}_{k}\right)=v \tau ; \mathfrak{q}_{i}, \mathfrak{q}_{j}, \mathfrak{q}_{k}$, v-ideals for $v$.

2. If $v\left(\mathfrak{q}_{i}\right)=m \tau(i>0)$ and $v\left(\mathfrak{q}_{i+1}\right)=u / t+v \tau$ then $v<m$ and $\mathfrak{q}_{i}=\mathfrak{q}_{j} \mathfrak{q}_{k}$ where $v\left(\mathfrak{q}_{j}\right)=(m-v) \tau, v\left(\mathfrak{q}_{k}\right)=v \tau ; \mathfrak{q}_{i}, \mathfrak{q}_{j}, \mathfrak{q}_{k}, v$-ideals for $v$.

3. If $v\left(\mathfrak{q}_{i}\right)=m / t$ ( $m$ an integer), $v\left(\mathfrak{q}_{i+1}\right)=u / t+v \tau$ and $v \neq 0$, then $u<m$ and $\mathfrak{q}_{i}=\mathfrak{q}_{j} \mathfrak{q}_{k}$ where $v\left(\mathfrak{q}_{j}\right)=(m-u) / t, v\left(\mathfrak{q}_{k}\right)=u / t, \mathfrak{q}_{i}, \mathfrak{q}_{j}, \mathfrak{q}_{k}$, v-ideals for $v$.

Proof. 1. We have to show that if $v\left(F\left(\alpha_{0}, \cdots, \alpha_{n}\right)\right) \geqq u / t+v \tau$ then $F\left(\alpha_{0}, \cdots, \alpha_{n}\right)=\sum a_{i}(x, y) b_{i}(x, y), a_{i}, b_{i} \in K[x, y]$, with $v\left(a_{i}\right) \geqq u / t, v\left(b_{i}\right) \geqq v \tau$, where $\sum$ is the summation of a finite number of products $a_{i} b_{i}$. This is clear for $v\left(F\left(\alpha_{0}, \cdots, \alpha_{n}\right)\right)$ sufficiently large; in fact, let $k_{0}, k_{1}, \cdots, k_{n}, l_{0}, l_{1}, \cdots, l_{n}$ be integers such that $k_{0} v(x) \geqq u / t, k_{1} v\left(f_{1}\right) \geqq u / t, \cdots, k_{n} v\left(f_{n}\right) \geqq u / t, l_{0} v(x) \geqq v \tau$, $l_{1} v\left(f_{1}\right) \geqq v \tau, \cdots, l_{n} v\left(f_{n}\right) \geqq v \tau$. If, now, $v\left(F\left(\alpha_{0}, \cdots, \alpha_{n}\right)\right) \geqq v\left(F\left(k_{0}+l_{0}, \cdots\right.\right.$, $\left.k_{n}+l_{n}\right)$ ) then $\alpha_{i} \geqq k_{i}+l_{i}$ for at least one $i, i=0,1, \cdots, n$; if $\alpha_{m} \geqq k_{m}+l_{m}$, say, then $F\left(\alpha_{0}, \cdots, \alpha_{n}\right) \equiv 0\left(f_{m} \exp k_{m}+l_{m}\right) \equiv 0\left(\mathfrak{q}_{j} \mathfrak{q}_{k}\right)$. Hence in the proof we may assume that all monomials of value greater than $v\left(F\left(\alpha_{0}, \cdots, \alpha_{n}\right)\right)$ are in $\mathfrak{q}_{j} \mathfrak{q}_{k}$. Moreover, if $v\left(F\left(\alpha_{0}, \cdots, \alpha_{n}\right)\right)=v\left(F\left(\beta_{0}, \cdots, \beta_{n}\right)\right)$ then $v\left(F\left(\alpha_{0}, \cdots, \alpha_{n}\right)-c F\left(\beta_{0}, \cdots, \beta_{n}\right)\right)>v\left(F\left(\alpha_{0}, \cdots, \alpha_{n}\right)\right)$ for some $c \in K$, so that $F\left(\alpha_{0}, \cdots, \alpha_{n}\right)-c F\left(\beta_{0}, \cdots, \beta_{n}\right)$ is a sum of monomials of value greater than $v\left(F\left(\alpha_{0}, \cdots, \alpha_{n}\right)\right)$, since every $v$-ideal $q$ has a basis consisting of monomials in $x, f_{1}, \cdots, f_{n}$. Hence it is sufficient to prove $F\left(\alpha_{0}, \cdots, \alpha_{n}\right)$ $=\sum a_{i} b_{i}$ for some monomial of value equal to $v\left(F\left(\alpha_{0}, \cdots, \alpha_{n}\right)\right)$. If $u / t+v \tau$ $=v\left(F\left(\beta_{0}, \cdots, \beta_{n-1}, v\right)\right)$ and $\alpha_{n} \geqq v$, then this is clear: $F\left(\alpha_{0}, \cdots, \alpha_{n-1}, \alpha_{n}\right)$ $=F\left(\alpha_{0}, \cdots, \alpha_{n-1}, \alpha_{n}-v\right) \cdot f_{n}^{0} \equiv 0\left(\mathfrak{q}_{j} \mathfrak{q}_{k}\right)$; if $\alpha_{n}<v$, then by the lemma we may assume $\alpha_{i} \geqq \beta_{i}, i=0,1, \cdots, n-1$, whence $F\left(\alpha_{0}, \cdots, \alpha_{n-1}, \alpha_{n}\right)=F\left(\alpha_{0}-\beta_{0}\right.$, $\left.\cdots, \alpha_{n-1}-\beta_{n-1}, \alpha_{n}\right) \cdot F\left(\beta_{0}, \cdots, \beta_{n-1}\right) \equiv 0\left(\mathfrak{q}_{k} \mathfrak{q}_{j}\right)$. This completes the proof.

2 . Let $[r]$ indicate the greatest integer equal to or less than the real number $r$. If $q$ is a $v$-ideal then $1+v(q)$ is the value of some $v$-ideal, whence $v\left(\mathfrak{q}_{i+1}\right)-v\left(\mathfrak{q}_{i}\right) \leqq 1$. Since $\tau=v\left(f_{n}\right) \geqq v\left(f_{2}\right)>d_{1} v\left(f_{1}\right) \geqq v(x)=1$, we must have $v \leqq m$. Moreover, $v=m$ is impossible; in fact, $v=m$ implies $u>0$ and $m \tau<[u+m t \tau] / t$ $<u / t+m \tau$. By (a) of Theorem $6,[u+m t \tau] / t$ is the value of a $v$-ideal; this is a contradiction. Hence $v<m$. The only monomial of value $m \tau$ in $\mathfrak{q}_{i}$ is $f_{n}^{m}$ $\left(=f_{n}^{m-v} f_{n}^{b}\right)$, which is clearly in $\mathfrak{q}_{j} \mathfrak{q}_{k}$. For monomials $F\left(\alpha_{0}, \cdots, \alpha_{n}\right)$ of value $\geqq u / t+v \tau$, we have, as in $1, F\left(\alpha_{0}, \cdots, \alpha_{n}\right)=\sum a_{i} b_{i}$, with $v\left(a_{i}\right) \geqq u / t, v\left(b_{i}\right)$ $\geqq v \tau$; since $u / t>(m-v) \tau, a_{i} \in \mathfrak{q}_{j}$, whence $F\left(\alpha_{0}, \cdots, \alpha_{n}\right) \equiv 0\left(\mathfrak{q}_{j} \mathfrak{q}_{k}\right)$. This completes the proof. 
3. Here $u / t+v \tau-1<m / t<u / t+v \tau$, since $v\left(\mathfrak{q}_{i+1}\right)-v\left(\mathfrak{q}_{i}\right)<1$ (equalities are excluded since $\tau$ is irrational and $v \neq 0$ ), so that not only $m>u$, but $(m-u) / t>\tau-1=v\left(f_{n}\right)-1$. Hence it follows from the lemma that there exist non-negative integers $\alpha_{i}, \beta_{i}$ with $\alpha_{i} \geqq \beta_{i}$ such that $v\left(F\left(\alpha_{0}, \cdots, \alpha_{n-1}\right)\right)$ $=m / t>u / t=v\left(F\left(\beta_{0}, \cdots, \beta_{n-1}\right)\right)$, whence at least one monomial of value $m / t$ is in $\mathfrak{q}_{j} \mathfrak{q}_{k}$. For monomials of value greater than $m / t$, the proof goes as in 2 .

THEOREM 12. Let $v\left(\mathfrak{q}_{i}\right) \geqq \tau$, v as in Theorem 11. The v-ideal $\mathfrak{q}_{i}$ is simple only if of the numbers $v\left(\mathfrak{q}_{i}\right), v\left(\mathfrak{q}_{i+1}\right)$, one is rational and the other an integral multiple of $\tau$.

Proof. If $v\left(\mathfrak{q}_{i}\right)=u / t+v \tau, u \neq 0, v \neq 0$, then $\mathfrak{q}_{i}$ is not simple by Theorem 11.1. If $v\left(\mathfrak{q}_{i}\right)=m \tau, v\left(\mathfrak{q}_{i+1}\right)=u / t+v \tau$, and $v \neq 0$ then $u \neq 0$ since $v<m$. Hence $\mathfrak{q}_{i}$ is not simple by Theorem 11.2. If $v\left(\mathfrak{q}_{i}\right)=m / t, m$ an integer, $t=t_{n-1}, v\left(\mathfrak{q}_{i+1}\right)=u / t+v \tau$, $u, v$ integers, and if, first, $v \neq 0$, then either $u \neq 0$ and $q_{i}$ is not simple by Theorem 11.3, or $u=0$ (and there is nothing to prove). If $v=0$ then by (a) of Theorem $6, v\left(\mathfrak{q}_{i+1}\right)=(m+1) / t$. Let $\mathfrak{q}_{k}$ be the first $v$-ideal after $\mathfrak{q}_{i}$ of value $u / t+v \tau$ such that $v \neq 0$. Then also $u \neq 0$. In fact, if $u=0$ then $v>1$. Moreover, $\tau>d_{n-1} v\left(f_{n-1}\right)=k / t, k$ an integer, whence $[t \tau] / t \geqq k / t=d_{n-1} v\left(f_{n-1}\right)$. Hence, by (a) of Theorem 6 , there exists a $v$-ideal $\mathfrak{q}_{j}$ with $v\left(\mathfrak{q}_{j}\right)=(v-1) \tau+[t \tau] / t . i<j<k$ since $0<\tau-[t \tau] / t<1 / t$. This is a contradiction, since $v-1 \neq 0$. Hence $u \neq 0$. Then $u<m$ and $\mathfrak{q}_{i}=\mathfrak{q}_{j} \mathfrak{q}_{l}$, with $v\left(\mathfrak{q}_{j}\right)=(m-u) / t, v\left(\mathfrak{q}_{l}\right)=u / t$. In fact, proceeding as in Theorem 11, we need only prove that some monomial of value $=v\left(q_{i}\right)$ $=m / t$ is in $\mathfrak{q}_{j} \mathfrak{q}_{l}$ (because at the same time this proves that some monomial of value $=v\left(\mathfrak{q}_{i^{\prime}}\right), i \leqq i^{\prime}<k$, is in $\left.\mathfrak{q}_{j} \mathfrak{q}_{l}\right)$. To prove this it is only necessary, by (a) of Theorem 6 , to show that $m / t-u / t>\tau-1$ (the proof then proceeding, by means of the lemma to Theorem 10, as in Theorem 10.3). If $v>1$ then $u / t+(v-1) \tau<m / t$, whence $m / t-u / t>(v-1) \tau \geqq \tau$. If $v=1$ then $u / t<\tau$. In fact, if $u / t>\tau$ then $u / t>d_{n-1} v\left(f_{n-1}\right)=k / t, k$ an integer, whence $(u-1) / t$ $\geqq d_{n-1} v\left(f_{n-1}\right)$; by (a) of Theorem $6,(u-1) / t+\tau$ is then the value of a $v$-ideal, whence $(u-1) / t+\tau<m / t$, or $u / t+\tau<(m+1) / t$ : this is a contradiction since we are in the case $v\left(\mathfrak{q}_{i+1}\right)=(m+1) / t<u / t+v \tau=u / t+\tau$. Since $v\left(\mathfrak{q}_{l}\right)=u / t<\tau$, we have $v\left(q_{l-1}\right)=u^{\prime} / t, u^{\prime}$ an integer $(l-1$ possibly $=0)$, and $u / t-u^{\prime} / t \leqq 1$. From $u^{\prime} / t+\tau<m / t$ follows: $m / t-u / t>\tau-1$. This completes the proof.

Let $v$ be as in Theorem $11, \mathfrak{q}_{1}, \mathfrak{q}_{2}, \cdots$ the Jordan sequence of 0 -dimensional $v$-ideals.

THEOREM 13. If of the numbers $v\left(\mathfrak{q}_{i}\right), v\left(\mathfrak{q}_{i+1}\right)$ one is rational and the other an integral multiple of $\tau$, then $\mathfrak{q}_{i}$ is simple.

Proof. a. Consider the case $v\left(\mathfrak{q}_{i}\right)=v \tau, v\left(\mathfrak{q}_{i+1}\right)=u / t, u$ an integer. Consider the ordered set of numbers, $v\left(\mathfrak{q}_{1}\right), v\left(\mathfrak{q}_{2}\right), \cdots v\left(\mathfrak{q}_{i-1}\right), v\left(\mathfrak{q}_{i}\right), v\left(\mathfrak{q}_{i+1}\right), \cdots$, that is, the numbers

$$
\cdots, m / t+n \tau, v \tau, u / t, \cdots \text {. }
$$


We shall show that there exists a rational rank 2 valuation $v_{2}$ of $\Sigma$ over $K$ with $v_{2}(x)=1, v_{2}(y)>0, v_{2}\left(f_{i}\right)=v\left(f_{i}\right), i=1, \cdots, n-1, v_{2}\left(f_{n}\right)=\tau^{\prime}$ such that the set of numbers: $v_{2}\left(\mathfrak{q}_{1}^{\prime}\right), v_{2}\left(\mathfrak{q}_{2}^{\prime}\right), \cdots, v_{2}\left(\mathfrak{q}_{i-1}^{\prime}\right), v_{2}\left(\mathfrak{q}_{i}^{\prime}\right), v_{2}\left(\mathfrak{q}_{i+1}^{\prime}\right), \cdots$, where $\mathfrak{q}_{1}^{\prime}, \mathfrak{q}_{2}^{\prime}, \ldots$ is the Jordan sequence of 0 -dimensional $v_{2}$-ideals, is the sequence:

$$
\cdots, m / t+n \tau^{\prime}, u / t, v \tau^{\prime}, \cdots .
$$

This implies that $\mathfrak{q}_{i}$ is simple. In fact, every $v_{2}$-ideal $q^{\prime}$ has a basis consisting of monomials $F\left(\alpha_{0}, \cdots, \alpha_{n}\right), \alpha_{i}$ non-negative integers, $i=0,1, \cdots, n-1$. This follows for the $v_{2}$-ideals exactly as for the $v$-ideals. We can now assert that $\mathfrak{q}_{1}=\mathfrak{q}_{1}^{\prime}, \cdots, \mathfrak{q}_{i}=\mathfrak{q}_{i}^{\prime}$. For example, to prove $\mathfrak{q}_{i}=\mathfrak{q}_{i}^{\prime}$, let $F\left(\alpha_{0}, \cdots, \alpha_{n}\right) \in \mathfrak{q}_{i}$, whence $v\left(F\left(\alpha_{0}, \cdots, \alpha_{n-1}\right)\right)+\alpha_{n} \tau \geqq v \tau$. Hence $v\left(F\left(\alpha_{0}, \cdots, \alpha_{n-1}\right)\right)+\alpha_{n} \tau$ is not among the first $i-1$ numbers of (1), hence $v_{2}\left(F\left(\alpha_{0}, \cdots, \alpha_{n-1}\right)\right)+\alpha_{n} \tau^{\prime}$ $\left(=v\left(F\left(\alpha_{0}, \cdots, \alpha_{n-1}\right)\right)+\alpha_{n} \tau^{\prime}\right)$ is not among the first $i-1$ numbers of $(2)$. Hence $v_{2}\left(F\left(\alpha_{0}, \cdots, \alpha_{n-1}\right)\right)+\alpha_{n} \tau^{\prime} \geqq u / t$, whence $F\left(\alpha_{0}, \cdots, \alpha_{n-1}, \alpha_{n}\right) \in q_{i}^{\prime}$. Thus $\mathfrak{q}_{i} \subseteq \mathfrak{q}_{i}^{\prime}$. Similarly $\mathfrak{q}_{i}^{\prime} \subseteq \mathfrak{q}_{i}$, whence $\mathfrak{q}_{i}=\mathfrak{q}_{i}^{\prime}$. Similarly, $\mathfrak{q}_{1}=\mathfrak{q}_{1}^{\prime}, \cdots, \mathfrak{q}_{i-1}=\mathfrak{q}_{i-1}^{\prime}$. Now suppose $\mathfrak{q}_{i}=\mathfrak{q}_{j} \mathfrak{q}_{k}$. Since $v\left(\mathfrak{q}_{i}\right)=v \tau$, we have $v\left(\mathfrak{q}_{j}\right), v\left(\mathfrak{q}_{k}\right)$ are integral multiples of $\tau$, say $v\left(\mathfrak{q}_{j}\right)=p \tau, v\left(\mathfrak{q}_{k}\right)=(v-p) \tau$. Since $j<i, k<i$, the numbers $p \tau^{\prime}$, $(v-p) \tau^{\prime}$ are the $j$ th and $k$ th numbers in the sequence $(2)$. Hence $v_{2}\left(q_{j}\right)=p \tau^{\prime}$, $v_{2}\left(\mathfrak{q}_{k}\right)=(v-p) \tau^{\prime}$, and $v_{2}\left(\mathfrak{q}_{i}\right)=v \tau^{\prime}$. This is a contradiction.

Thus it remains to prove the existence of the valuation $v_{2}$. Suppose that there exists an irrational number $\tau^{\prime}$ such that the set of positive numbers $\left\{\alpha_{0}+\alpha_{1} v\left(f_{1}\right)+\cdots+\alpha_{n-1} v\left(f_{n-1}\right)+\alpha_{n} \tau^{\prime}\right\}, \alpha_{i}$ non-negative integers, ordered according to magnitude, is the sequence (2); then $\tau^{\prime}>\tau$ is immediately implied by (2) and (1): $\tau^{\prime}>u / v t>\tau$. The existence of the valuation $v_{2}$ is then given by Theorem 7 . Thus it remains to find $\tau^{\prime}$.

Consider the sequence obtained by multiplying (1) by $t=t_{n-1}$, that is, the sequence

$$
\cdots, m+n(t \tau), v(t \tau), u, \cdots .
$$

This set of numbers will be contained, in the same order, in the set of positive numbers $\{g+h(t \tau)\}, g, h$ non-negative integers, ordered according to magnitude, and, in fact, (1) can be obtained from that sequence by first dropping the numbers $k+l(t \tau)$ in which $k$ is not of the form $t\left(\alpha_{0}+\alpha_{1} v\left(f_{1}\right)+\cdots\right.$ $\left.+\alpha_{n-1} v\left(f_{n-1}\right)\right), \alpha_{0}, \alpha_{1}, \cdots, \alpha_{n-1}$ non-negative integers, and then dividing the sequence by $t$.

Now we assert that the numbers $v(t \tau), u$ which are successive in $\left(1^{\prime}\right)$ are also successive in the ordered set of numbers $\{g+h(t \tau)\}$. In fact, let $k+l(t \tau)$ be the number immediately following $v(t \tau)$ in the sequence $\{g+h(t \tau)\}$. Since $v(t \tau)<[v(t \tau)+1]<v(t \tau)+1$, necessarily $0<k+l(t \tau)-v(t \tau)<1$, whence $l<v$, and $k>(v-l) t \tau \geqq t \tau=t v\left(f_{n}\right)$. Hence by (a) of Theorem $6, k$ is of the form $t\left(\alpha_{0}+\alpha_{1} v\left(f_{1}\right)+\cdots+\alpha_{n-1} v\left(f_{n-1}\right)\right)$. Hence $k / t+l \tau$ succeeds $v \tau$ in (1), whence $l=0$ and $k=u$.

Let then the sequence of numbers $\{g+h(t \tau)\}$ be 


$$
1, \cdots, p+q(t \tau), v(t \tau), u, \cdots .
$$

From our consideration of valuations given by $y=x^{\tau}$, there exists an irrational number $\tau^{\prime}$ such that the ordered set of numbers $\left\{g+h\left(t \tau^{\prime}\right)\right\}$ is the sequence

$$
1, \cdots, p+q\left(t \tau^{\prime}\right), u, v\left(t \tau^{\prime}\right), \cdots .
$$

Dropping from this sequence the numbers $k+l\left(t \tau^{\prime}\right)$ in which $k$ is not of the form $t\left(\alpha_{0}+\alpha_{1} v\left(f_{1}\right)+\cdots+\alpha_{n-1} v\left(f_{n-1}\right)\right)$, and dividing by $t$, we obtain the sequence (2). This completes the proof in case a.

b. This is the case: $v\left(\mathfrak{q}_{i}\right)=u / t, v\left(\mathfrak{q}_{i+1}\right)=v \tau$. The proof is almost the same. Here the sequence $v\left(\mathfrak{q}_{1}\right), v\left(\mathfrak{q}_{2}\right), \cdots, v\left(\mathfrak{q}_{i-1}\right), v\left(\mathfrak{q}_{i}\right), v\left(\mathfrak{q}_{i+1}\right), \cdots$ is the sequence:

$$
\cdots, m / t+n \tau, u / t, v \tau, \cdots .
$$

Multiplying (1) by $t$, we get the sequence

$$
\cdots, m+n(t \tau), u, v(t \tau), \cdots
$$

which we wish to compare with the ordered set of numbers $\{g+h(t \tau)\}, g, h$ non-negative integers; namely, we wish to show that $u$ and $v(t \tau)$ are successive in the set $\{g+h(t \tau)\}$, ordered according to magnitude. In fact, let $k+l(t \tau)$ be the number immediately preceding $v(t \tau)$ in the set $\{g+h(t \tau)\}$. Then $l<v$ and $k+1+l(t \tau)>v(t \tau)$ (clearly $k+1+l(t \tau) \geqq v(t \tau)$; equality is excluded since $k+1$ is an integer, while $(v-l) t \tau$ is not), so that $k+1>(v-l) t \tau \geqq t \tau>t d_{n-1} v\left(f_{n-1}\right)$, whence $k \geqq t d_{n-1} v\left(f_{n-1}\right)$ since $k, t d_{n-1} v\left(f_{n-1}\right)$ are integers. Hence, as above, $l=0$, $k=u$, and we can find an irrational number $\tau^{\prime}$ such that the ordered set of numbers $\left\{\alpha_{0}+\alpha_{1} v\left(f_{1}\right)+\cdots+\alpha_{n-1} v\left(f_{n-1}\right)+\alpha_{n} \tau^{\prime}\right\}$ is the sequence

$$
\cdots, m / t+n \tau^{\prime}, v \tau^{\prime}, u / t, \cdots \text {. }
$$

However, it does not follow as in (a) that there exists the required valuation $v_{2}$. For $v>1$, however, it does follow. In fact, $(v-1) \tau+d_{n-1} v\left(f_{n-1}\right) \leqq u / t$, since $u / t$ and $v \tau$ are successive in (1), and if $v>1$ then $(v-1) \tau+d_{n-1} v\left(f_{n-1}\right)<u / t$, equality being excluded since $u / t$ is rational while $(v-1) \tau+d_{n-1} v\left(f_{n-1}\right)$ is not. Hence, by (2), $(v-1) \tau^{\prime}+d_{n-1} v\left(f_{n-1}\right)<v \tau^{\prime}$; hence $\tau^{\prime}>d_{n-1} v\left(f_{n-1}\right)$. Thus for $v>1$ the required valuation $v_{2}$ exists by Theorem 8.3.

For $v=1$, the fact that $\mathfrak{q}_{i}$ is simple is given by Corollary 3 of Theorem 10 . This completes the proof.

Corollary. If $v\left(\mathfrak{q}_{i}\right)=u / t, v\left(\mathfrak{q}_{i+1}\right)=v \tau$ or if $v\left(\mathfrak{q}_{i}\right)=v \tau, v\left(\mathfrak{q}_{i+1}\right)=u / t$ then $(u, v)=1$ and $u / v$ is an approximant or quasi approximant to $t \tau$; and conversely.

Proof. The direct statement follows by Lemma 1 of Theorem 4 from the fact that $u, v(t \tau)$ (or $v(t \tau), u$ ) are successive in the ordered set of numbers $\{g+h(t \tau)\}, g, h$ non-negative integers. Conversely, let $u / v$ be an approximant or quasi approximant to $t \tau$. Since $v$ is of type $n>1, \tau=v\left(f_{n}\right) \geqq v\left(f_{2}\right)>d_{1} v\left(f_{1}\right) \geqq 1$, since $d_{1} v\left(f_{1}\right)$ is a positive integer. Hence $v / u$ is certainly an approximant or 
quasi approximant to $1 / t \tau(<1)$. Hence by Lemma 1 of Theorem $4, u, v(t \tau)$ (or $v(t \tau), u$ ) are successive in the set $\{g+h(t \tau)\}$. Now $\tau>d_{n-1} v\left(f_{n-1}\right)=k / t, k$ an integer, whence $u \geqq[t \tau] \geqq k=t d_{n-1} v\left(f_{n-1}\right)$. Hence by (a) of Theorem $6, u / t$ is an element of (1); that is, $u / t, v \tau$ (or $v \tau, u / t$ ) are successive in (1). This completes the proof.

7. The quadratic transformation $T$ and rational rank 2 valuations. As already indicated, Theorem 9 allows us in the study of valuation ideals to restrict ourselves to rational rank 2 valuations, at least if the study is confined to the ring $\mathfrak{D}=K[x, y]$. We wish now to see to what extent the same restriction can be made when the transformation $T$ and the ring $\mathfrak{O}^{\prime}=K\left[x^{\prime}, y^{\prime}\right]$ are also involved.

Let, then, $v$ be a 0 -dimensional valuation in which, without loss of generality, we assume $v(y)>v(x)>0 . T$ is the transformation:

$$
T: \quad x^{\prime}=x, \quad y^{\prime}=y / x ; \quad x=x^{\prime}, \quad y=x^{\prime} y^{\prime} .
$$

Let $f_{1}(x, y), f_{2}(x, y), \cdots$ be the (finite or infinite) sequence of polynomials introduced in Theorem 6. From these polynomials, we wish to derive polynomials $f_{1}^{\prime}\left(x^{\prime}, y^{\prime}\right), f_{2}^{\prime}\left(x^{\prime}, y^{\prime}\right), \cdots$, in the ring $K\left[x^{\prime}, y^{\prime}\right]$, satisfying Theorem 6 for $v$. In accordance with (b) of Theorem 6 , let $t_{i-1}=\operatorname{deg}_{y} f_{i}$.

Lemma. $f_{i}(x, y)=f_{i}\left(x^{\prime}, x^{\prime} y^{\prime}\right) \equiv 0\left(x^{\prime} \exp t_{i-1}\right), f_{i}\left(x^{\prime}, x^{\prime} y^{\prime}\right) \not \equiv 0\left(x^{\prime} \exp t_{i-1}+1\right)$.

Proof. The proof is by induction on $i$. For $i=1, \operatorname{deg}_{y} f_{1}=1$, and $f_{1}$ is monic in $y$, whence $f_{1}(x, y)=f_{1}\left(x^{\prime}, x^{\prime} y^{\prime}\right) \not \equiv 0\left(x^{\prime 2}\right)$; since $v\left(f_{1}\right)>0, f_{1}(x, y)=f_{1}\left(x^{\prime}, x^{\prime} y^{\prime}\right)$ $\equiv 0\left(x^{\prime}\right)$. For $i>1, \operatorname{deg}_{y}\left(f_{i}(x, y)-f_{i-1}^{d(t-1)}(x, y)\right)<\operatorname{deg}_{y} f_{i}(x, y)$, whence, by (c) of Theorem $6, f_{i}-f_{i-1}^{d(t-1)}$ is a sum of monomials in $x, f_{1}, \cdots, f_{i-1}$ with distinct values:

$$
f_{i}-f_{i-1}^{d(i-1)}=\sum c\left(\alpha_{0}, \cdots, \alpha_{i-1}\right) F\left(\alpha_{0}, \cdots, \alpha_{i-1}\right), c\left(\alpha_{0}, \cdots, \alpha_{i-1}\right) \in K,
$$

$0 \leqq \alpha_{0}, 0 \leqq \alpha_{k}<d_{k}, k=1, \cdots, i-1$. Hence

$$
v\left(F\left(\alpha_{0}, \cdots, \alpha_{i-1}\right) \geqq v\left(f_{i}-f_{i-1}^{d(i-1)}\right)=v\left(f_{i-1}^{d(i-1)}\right)\right.
$$

for each term of the sum $\sum$; that is,

$$
\alpha_{0}+\alpha_{1} v\left(f_{1}\right)+\cdots+\alpha_{i-1} v\left(f_{i-1}\right) \geqq d_{i-1} v\left(f_{i-1}\right) .
$$

By (3) and (5) of Theorem 6,

$$
d_{i-1} \operatorname{deg}_{y} f_{i-1}=d_{1} \operatorname{deg}_{y} f_{1}+\left(d_{2}-1\right) \operatorname{deg}_{y} f_{2}+\cdots+\left(d_{i-1}-1\right) \operatorname{deg}_{y} f_{i-1}
$$

and

$$
d_{i-1} v\left(f_{i-1}\right)>d_{1} v\left(f_{1}\right)+\left(d_{2}-1\right) v\left(f_{2}\right)+\cdots+\left(d_{i-1}-1\right) v\left(f_{i-1}\right) .
$$

A fortiori,

$$
d_{i-1}\left(v\left(f_{i-1}\right)-\operatorname{deg}_{y} f_{i-1}\right)>\alpha_{1}\left(v\left(f_{1}\right)-\operatorname{deg}_{y} f_{1}\right) \cdots+\alpha_{i-1}\left(v\left(f_{i-1}\right)-\operatorname{deg}_{y} f_{i-1}\right) .
$$


Combining this with (1) we have,

$$
\alpha_{0}+\alpha_{1} \operatorname{deg}_{y} f_{1}+\cdots+\alpha_{i-1} \operatorname{deg}_{y} f_{i-1}>d_{i-1} \operatorname{deg}_{y} f_{i-1}=\operatorname{deg}_{y} f_{i} .
$$

Hence $f_{i}(x, y)-f_{i-1}^{d(i-1)}(x, y)=f_{i}\left(x^{\prime}, x^{\prime} y^{\prime}\right)-f_{i-1}^{d(i-1)}\left(x^{\prime}, x^{\prime} y^{\prime}\right) \equiv 0\left(x^{\prime} \exp t_{i-1}+1\right)$, by induction. Also by induction,

$$
f_{i-1}^{d(i-1)}(x, y)=f_{i-1}^{d(i-1)}\left(x^{\prime}, x^{\prime} y^{\prime}\right) \equiv 0\left(x^{\prime} \exp t_{i-1}\right)
$$

but

$$
f_{i-1}^{d(i-1)}\left(x^{\prime}, x^{\prime} y^{\prime}\right) \not \equiv 0\left(x^{\prime} \exp t_{i-1}+1\right)
$$

whence

$$
f_{i}(x, y)=f_{i}\left(x^{\prime}, x^{\prime} y^{\prime}\right) \equiv 0\left(x^{\prime} \exp t_{i-1}\right),
$$

but

$$
f_{i}\left(x^{\prime}, x^{\prime} y^{\prime}\right) \not \equiv 0\left(x^{\prime} \exp t_{i-1}+1\right) .
$$

This completes the proof. We also make the remark (already proved) that if $0 \leqq \alpha_{k}<d_{k}, k=1, \cdots, i-2$, then $v\left(f_{i-1}\right)-\operatorname{deg}_{y} f_{i-1}>\alpha_{1}\left(v\left(f_{1}\right)-\operatorname{deg}_{y} f_{1}\right)+\cdots$ $+\alpha_{i-2}\left(v\left(f_{i-2}\right)-\operatorname{deg}_{y} f_{i-2}\right)$. Note also the following : $\operatorname{deg}_{y} f_{i}=$ subdegree $f_{i}(=$ degree of the leading form of $f_{i}$ ).

Theorem 14. We place

$$
f_{i}^{\prime}\left(x^{\prime}, y^{\prime}\right)=f_{i}\left(x^{\prime}, x^{\prime} y^{\prime}\right) / x^{\prime} \exp t_{i-1} .
$$

The elements $f_{i}^{\prime}\left(x^{\prime}, y^{\prime}\right)$ are polynomials in $K\left[x^{\prime}, y^{\prime}\right]$ and satisfy Theorem 6 for $v$.

Proof. That the $f_{i}\left(x^{\prime}, y^{\prime}\right)$ are polynomials, and, moreover, monic in $y^{\prime}$, is the content of the lemma. We proceed now by induction on $i$. Note that $v\left(f_{i}\right)-v\left(f_{i}^{\prime}\right)$ is an integral multiple of $v(x)$, in fact equals $t_{i-1} v(x)$. Since $f_{1}^{\prime}$ is monic, of degree 1 in $y^{\prime}$, it follows as in Theorem 6 , that any polynomial $g\left(x^{\prime}, y^{\prime}\right) \in K\left[x^{\prime}, y^{\prime}\right]$ with $v(g)<v\left(f_{1}^{\prime}\right)$ has an integral multiple of $v(x)$ as value; that is, (2) of Theorem 6 is satisfied for $i=1$. For $i>1$, (3) is trivial since $\operatorname{deg}_{y} f_{i}=\operatorname{deg}_{y} f_{i}^{\prime} ;$ (4) follows directly from the remark that $v\left(f_{i}^{\prime}\right)-v\left(f_{i}\right)$ is an integral multiple of $v(x)$, and the fact that all the groups $D_{i}$ involved contain the group of integral multiples of $v(x)$. (We assume by induction that $\Delta_{i-1}=\Delta_{i-1}^{\prime}$.) As for (5),

$$
\begin{aligned}
v\left(f_{i}^{\prime}\right) & =v\left(f_{i}\right)-t_{i-1} v(x)>d_{i-1} v\left(f_{i-1}\right)-t_{i-1} v(x) \\
& =d_{i-1}\left(v\left(f_{i-1}^{\prime}\right)+t_{i-2} v(x)\right)-t_{i-1} v(x)=d_{i-1} v\left(f_{i-1}^{\prime}\right) .
\end{aligned}
$$

Thus it remains to prove (2). Explicitly stated, we have to prove that if $g\left(x^{\prime}, y^{\prime}\right) \in K\left[x^{\prime}, y^{\prime}\right]$ has value less than the value of $f_{i}^{\prime}$ then $v(g)$ $=v\left(F^{\prime}\left(\alpha_{0}, \cdots, \alpha_{i-1}\right)\right)$ for some integers $\alpha_{0}, \alpha_{1}, \cdots, \alpha_{i-1}$, where $F^{\prime}$ is a monomial in the $f_{\xi}^{\prime}$. Since $f_{i}^{\prime}$ is monic in $y$ and $v(g)<v\left(f_{\imath}^{\prime}\right)$, reducing $g\left(x^{\prime}, y^{\prime}\right)$ mod $f_{i}^{\prime}\left(x^{\prime}, y^{\prime}\right)$, we may assume $m=\operatorname{deg}_{y^{\prime}} g\left(x^{\prime}, y^{\prime}\right)<\operatorname{deg}_{y^{\prime}} f_{i}^{\prime}$. But then 


$$
g\left(x^{\prime}, y^{\prime}\right)=g(x, y / x)=g^{\prime}(x, y) / x^{m}, g^{\prime}(x, y) \in K[x, y] .
$$

Hence $v\left(g^{\prime}\right)=m v(x)+v(g)<t_{i-1} v(x)+v\left(f_{i}^{\prime}\right)=v\left(f_{i}\right)$, whence $v\left(g^{\prime}\right) \in \Delta_{i-1}$. Since $v\left(g^{\prime}\right)-v(g)$ is an integral multiple of $v(x),(2)$ now follows. Note finally that if the sequence $f_{1}, f_{2}, \ldots$ is finite, then the sequence $f_{1}^{\prime}, f_{2}^{\prime}, \ldots$ is finite, and of the same length, as follows at once by considering the 1-1 correspondence with the groups $\Delta_{i}$. This completes the proof.

We now derive a theorem which is a continuation, in a sense, to Theorem 9. Let $v_{1}$ be the rational rank 2 valuation introduced for $v$ in Theorem 9 . If $\tau$ is the least irrational $v_{1}$-value assumed by elements in $\mathscr{D}$ then we have seen by Corollary 1 to Theorem 9 that for certain integers $s$ it is true that the $v_{1}$-ideals of value less than $s \tau$ are the first $v$-ideals for $v$. Note that in Theorem 9 we now have $\tau>1$, since we are assuming $v(y)>v(x)>0$.

THeOREM 15. Let $v$ and $v_{1}$ be as in Theorem 9; let $\tau$ be the least irrational $v_{1}$-value assumed by elements in $\mathfrak{D}$, and let $\tau^{\prime}$ be the least irrational $v_{1}$-value assumed by elements in $\mathfrak{D}^{\prime}$. If for some integer $s$ the $v_{1}$-ideals in $\mathfrak{D}$ of $v_{1}$-value less than $s \tau$ are the first $v$-ideals for $v$, then also in $\mathfrak{D}^{\prime}$ the $v_{1}$-ideals of $v_{1}$-value less than $s \tau^{\prime}$ are the first $v$-ideals for $v$.

Proof. Let $f_{1}, \cdots, f_{j+1}$ be the polynomials of Theorem 9 , and consider the elements $F\left(\alpha_{0}, \cdots, \alpha_{j}, \alpha_{j+1}\right), 0 \leqq \alpha_{0}, 0 \leqq \alpha_{i}<d_{i}, i=1, \cdots, j, 0 \leqq \alpha_{j+1}<s$. Any two of these have distinct $v_{1}$-value and, by Corollary 2 of Theorem 9 , the integer $s$ is bounded in such manner that they also have distinct $v$-value. We may therefore order these elements according to their $v$ - and $v_{1}$-values, obtaining the simple sequences

$$
\begin{aligned}
& u_{1}, u_{2}, \cdots, \\
& w_{1}, w_{2}, \cdots,
\end{aligned}
$$

respectively. Let $\mathfrak{q}_{1}, \mathfrak{q}_{2}, \cdots ; \mathfrak{q}_{1}^{*}, \mathfrak{q}_{2}{ }^{*}, \cdots$ be the Jordan sequences of 0 -dimensional valuation ideals for $v$ and $v_{1}$ respectively. If $v_{1}\left(w_{k}\right)<v_{1}\left(f_{j+1}^{s}\right)<v_{1}\left(w_{k+1}\right)$ then, as in Corollary 2 of Theorem $9, u_{i}=w_{i}, i=1, \cdots, k$. In the ring $\mathfrak{D}^{\prime}=K\left[x^{\prime}, y^{\prime}\right]$ consider the elements $F^{\prime}\left(\alpha_{0}, \cdots, \alpha_{j}, \alpha_{j+1}\right)$, with the same limitations on the $\alpha_{i}$. Now if

$$
v\left(F^{\prime}\left(\alpha_{0}, \cdots, \alpha_{j+1}\right)\right)<v\left(F^{\prime}\left(\beta_{0}, \cdots, \beta_{j+1}\right)\right)<s v\left(f_{j+1}^{\prime}\right)
$$

then

$$
\begin{aligned}
v\left(F\left(\alpha_{0}+m-\sigma_{1}, \alpha_{1}, \cdots, \alpha_{j+1}\right)\right) & <v\left(F\left(\beta_{0}+m-\sigma_{2}, \beta_{1}, \cdots, \beta_{j+1}\right)\right) \\
& <s v\left(f_{j+1}\right)
\end{aligned}
$$

where $m=s \cdot \operatorname{deg}_{y} f_{j+1}, \sigma_{1}=\operatorname{deg}_{y} F\left(0, \alpha_{1}, \cdots, \alpha_{j+1}\right), \sigma_{2}=\operatorname{deg}_{y} F\left(0, \beta_{1}, \cdots, \beta_{j+1}\right)$. Since $\sigma_{1}<m$ and $\sigma_{2}<m$, the elements evaluated in (4) are in $K[x, y]$, whence the inequality (4) holds with $v$ replaced by $v_{1}$, whence it follows that (3) holds with $v$ replaced by $v_{1}$. Likewise, any two distinct $F^{\prime}\left(\alpha_{0}, \cdots, \alpha_{j+1}\right)$ have dis- 
tinct $v$-value. Hence the elements $F^{\prime}\left(\alpha_{0}, \cdots, \alpha_{j+1}\right)$ can be ordered according to their $v$ and $v_{1}$-values to yield the sequences

$$
\begin{aligned}
& u_{1}^{\prime}, u_{2}^{\prime}, \cdots, \\
& w_{1}^{\prime}, w_{2}^{\prime}, \cdots,
\end{aligned}
$$

respectively, and if $v_{1}\left(w_{l}^{\prime}\right)<v_{1}\left(f_{j+1}^{s}\right)<v\left(w_{l+1}^{\prime}\right)$ then $u_{i}^{\prime}=w_{i}^{\prime}, i=1, \cdots, l$. It follows now in a manner several times employed that the first $l$ valuation ideals in $D^{\prime}$ for $v_{1}$ coincide with the first $l$ for $v$. This completes the proof.

Theorem 15, like Theorem 9, will be used to replace a given valuation by a rational rank 2 valuation.

CoROllaRy. Theorem 15 also holds for a rational rank 2 valuation $v$ (of type not less than $j+1$ ) having $f_{1}, \cdots, f_{j+1}$ as the first $j+1$ associated polynomials, and such that $v\left(f_{i}\right)=v_{1}\left(f_{i}\right), i=1, \cdots, j$.

8. The transform of a simple $v$-ideal, general case. We come now to the proof that the transform of a simple $v$-ideal is simple. As usual we assume $v(y)>v(x)>0$; and $T$ is the transformation

$$
T: \quad x^{\prime}=x, \quad y^{\prime}=y / x ; \quad x=x^{\prime}, \quad y=x^{\prime} y^{\prime} .
$$

TheOREM 16. The transform of a v-ideal $\mathfrak{q}$ is a valuation ideal for $v$.

Proof. If $v$ is not already of rational rank 2 , let $v_{1}$ be a rational rank 2 valuation, as given in Theorem 9 , such that $v_{1}(\mathfrak{q})<s v\left(f_{j+1}\right)$ and such that the $v_{1}$-ideals of $v_{1}$-value less than $s v_{1}\left(f_{j+1}\right)$ are also the first $v$-ideals for $v$. We assert that $v_{1}(T(\mathfrak{q}))<s v_{1}\left(f_{j+1}^{\prime}\right)$. In fact, the ideal $\mathfrak{q}$ has a basis consisting of $f_{j+1}^{s}$ and of elements $F\left(\alpha_{0}, \cdots, \alpha_{j}, \alpha_{j+1}\right), 0 \leqq \alpha_{0}, 0 \leqq \alpha_{i}<d_{i}, i=1, \cdots, j, 0 \leqq \alpha_{j+1}<s$. Placing

$$
h=h(\mathfrak{q})=\min \left\{\alpha_{0}+\sum \alpha_{i} \operatorname{deg}_{y} f_{i}\right\}
$$

over the elements of this basis, we have $v_{1}(T(\mathfrak{q}))=v_{1}(\mathfrak{q})-h$. Clearly $h \leqq \operatorname{deg}_{y} f_{j+1}^{s}$. If equality holds, the assertion follows at once. Supposing $h<\operatorname{deg}_{y} f_{j+1}^{s}$, then for at least one of the basis elements $F\left(\alpha_{0}, \cdots, \alpha_{j+1}\right)$, it is true that

$$
v\left(F\left(\alpha_{\theta}, \cdots, \alpha_{j+1}\right)\right)-\left(\alpha_{0}+\sum \alpha_{i} \operatorname{deg}_{y} f_{i}\right) \geqq v(T(\mathfrak{q})),
$$

whence the assertion follows from the remark at the end of the lemma to Theorem 14. Hence, by Theorem 15 , if $T(\mathfrak{q})$ is a $v_{1}$-ideal it is also a $v$-ideal, and we may pass from $v$ to $v_{1}$, that is we may assume without loss of generality that $v$ is rational rank 2.

We now prove a lemma.

Lemma. Let $0 \leqq \alpha_{i}<d_{i}, i=1, \cdots, j ; 0 \leqq \alpha_{j+1}$. If the subdegree $k$ of $F\left(0, \alpha_{1}, \cdots, \alpha_{j+1}\right)$ is equal to or greater than $h=h(q)$ then

$$
v\left(F\left(0, \alpha_{1}, \cdots, \alpha_{j+1}\right)\right)-k \geqq v(\mathfrak{q})-h .
$$


Proof. The proof is by induction on $k$. In fact, let $k>h$. Since subd $f_{1}=1$ and $v\left(f_{1}\right)>1=v(x)$ we have, if $\alpha_{1} \neq 0$,

$v\left(F\left(0, \alpha_{1}, \cdots, \alpha_{j+1}\right)\right)-k>v\left(F\left(0, \alpha_{1}-1, \alpha_{2}, \cdots, \alpha_{j+1}\right)\right)-(k-1) \geqq v(\mathfrak{q})-h$,

by induction. If $\alpha_{1}=0$, let $\alpha_{1}=\cdots=\alpha_{i-1}=0, \alpha_{i} \neq 0$; then

$$
\begin{aligned}
v\left(F\left(0, \alpha_{1}, \cdots, \alpha_{j+1}\right)\right)-k \\
\quad>v\left(F\left(0, d_{1}-1, \cdots, d_{i-1}-1, \alpha_{i}-1, \alpha_{i+1}, \cdots, \alpha_{j+1}\right)\right)-(k-1) \\
\quad \geqq v(\mathrm{q})-h,
\end{aligned}
$$

by induction. Thus it remains to prove the lemma for $k=h$. In the case $k=h$, let $F\left(\beta_{0}, \cdots, \beta_{j+1}\right)$ be an element of $q$ of subdegree $h$. Clearly $\alpha_{j+1} \geqq \beta_{j+1}$. If $\alpha_{i}=\beta_{i}, i=1, \cdots, j+1$, then $\beta_{0}=0$ and the lemma is trivial. Otherwise, let $\alpha_{i+1}=\beta_{i+1}, \cdots, \alpha_{j+1}=\beta_{j+1}$ but $\alpha_{i}>\beta_{i}$. Then clearly,

$$
\begin{array}{r}
v\left(F\left(0, \alpha_{1}+d_{1}-\beta_{1}, \alpha_{2}+d_{2}-\beta_{2}-1, \cdots, \alpha_{i}+d_{i}-\beta_{i}-1, \alpha_{i+1}-\beta_{i+1}-1\right)\right. \\
>\beta_{0} v(x),
\end{array}
$$

since both terms evaluated have like subdegree, and $v(y)>v(x)$. The lemma is an immediate consequence.

To proceed with the proof of the theorem, let $g\left(x^{\prime}, y^{\prime}\right) \in D^{\prime}$, with $v(g)$ $\geqq v(T(\mathfrak{q}))$ : to prove $g \in T(\mathfrak{q})$. We can write $g\left(x^{\prime}, y^{\prime}\right)=\sum c\left(\alpha_{0}, \cdots, \alpha_{j+1}\right)$ - $F^{\prime}\left(\alpha_{0}, \cdots, \alpha_{j+1}\right)$, where $\sum$ is a finite sum of terms with distinct value. Thus it suffices to assume $g=F^{\prime}\left(\alpha_{0}, \cdots, \alpha_{j+1}\right)$, where $0 \leqq \alpha_{0}, 0 \leqq \alpha_{i}<d_{i}, i=1, \cdots, j$, $0 \leqq \alpha_{j+1}$. Clearly, if $\alpha_{0}$ is sufficiently large then $g \in T(\mathfrak{q})$; in fact, if $v\left(x^{\alpha(0)}\right) \geqq v(\mathfrak{q})$ then $x^{\prime \alpha(0)}$ arises from $x^{\alpha(0)+h}$, and $g$ is a multiple of $x^{\prime \alpha(0)}$. Thus we may assume that if $h\left(x^{\prime}, y^{\prime}\right) \equiv 0\left(x^{\prime \alpha(0)+1}\right)$ and $v(h) \geqq v(T(\mathfrak{q}))$ then $h\left(x^{\prime}, y^{\prime}\right) \in T(\mathfrak{q})$. We now make an induction on $k=\operatorname{subd} F^{\prime}\left(0, \alpha_{1}, \cdots, \alpha_{j+1}\right)$. If $k \leqq h$ then $g\left(x^{\prime}, y^{\prime}\right)$ arises from $F\left(\alpha_{0}+h-k, \alpha_{1}, \cdots, \alpha_{j+1}\right)$. Let, now, $k>h$. If $\alpha_{1}>0$ then by the lemma $F^{\prime}\left(\alpha_{0}, \alpha_{1}-1, \alpha_{2}, \cdots, \alpha_{j+} 1\right)$ has value not less than $T(q)$, and hence is in $T(q)$ by induction, whence also $g \in T(q)$. If $\alpha_{1}=0$, let $\alpha_{1}=\cdots=\alpha_{i-1}=0$, $\alpha_{i} \neq 0$. Then

$$
\begin{aligned}
& F^{\prime}\left(\alpha_{0}, 0, \cdots, 0, \alpha_{i}, \cdots, \alpha_{j+1}\right) \\
& =f_{1}^{\prime} \cdot F^{\prime}\left(\alpha_{0}, d_{1}-1, \cdots, d_{i-1}-1, \alpha_{i}-1, \alpha_{i+1}, \cdots, \alpha_{j+1}\right) \\
& \quad+x^{\prime \alpha(0)+1} h\left(x^{\prime}, y^{\prime}\right),
\end{aligned}
$$

where $h\left(x^{\prime}, y^{\prime}\right) \in \mathfrak{D}^{\prime}$. The first term on the right is a multiple of $F^{\prime}\left(\alpha_{0}, d_{1}-1, \cdots, d_{i-1}-1, \alpha_{i}-1, \alpha_{i+1}, \cdots, \alpha_{j+1}\right)$, which by the lemma has value not less than $T(q)$, and hence is in $T(q)$ by induction. Hence also the second term has value not less than $T(q)$, and since it is divisible by $x^{\prime \alpha(0)+1}$ it also is in $T(\mathfrak{q})$. Thus $g \in T(\mathfrak{q})$. This completes the proof.

THEOREM 17. The transform by $T$ of the ith simple v-ideal $\mathfrak{P}_{i}$ is the $(i-1)$ th simple v-ideal $\mathfrak{B}_{i-1}^{\prime}$. 
Proof. As in the previous theorem, if $v$ is not already of rational rank 2 then there exists a rational rank 2 valuation $v_{1}$ such that the $v$-ideals up to and including $\mathfrak{P}_{i}$ coincide with the $v_{1}$-ideals up to and including $\mathfrak{P}_{i}$ and such that the $v$-ideals in $\mathfrak{D}^{\prime}$ up to and including $T\left(\mathfrak{B}_{i}\right)$ coincide with the $v_{1}$-ideals up to and including $T\left(\mathfrak{B}_{i}\right)$. Hence $\mathfrak{B}_{i}$ is also the $i$ th simple $v_{1}$-ideal, and if $T\left(\mathfrak{B}_{i}\right)$ is the $(i-1)$ th simple $v_{1}$-ideal then it is also the $(i-1)$ th simple $v$-ideal for $v$. Thus we may pass from $v$ to $v_{1}$; that is, without loss of generality we may assume $v$ to be of rational rank 2 .

Let $n$ be the type of $v$ with respect to the ring $K[x, y] ; f_{1}(x, y), \cdots, f_{n}(x, y)$, the polynomials introduced in Theorem 6 for $v$, and let $v\left(f_{n}\right)=\tau$. The proof is by induction on $n$. For $n=1$, the proof has already been given in Theorem 5. For $n>1$, every $v$-ideal of value equal to or less than $d_{n-1} v\left(f_{n-1}\right)$ belongs also, by Theorem 10, to a rational rank 2 valuation $v^{\prime}$ of type $n-1$ with respect to $K[x, y]$ : and moreover $v^{\prime}$ exists such that $\mathfrak{B}_{i}$ is the $i$ th simple $v^{\prime}$-ideal. (Here also, by corollary, Theorem 15 , if $T\left(\mathfrak{B}_{i}\right)$ is the $(i-1)$ th simple $v^{\prime}$-ideal, then it is the $(i-1)$ th simple $v$-ideal.) Thus we may assume $v\left(\mathfrak{B}_{i}\right)>d_{n-1} v\left(f_{n-1}\right)$ by induction. If $v\left(\mathfrak{B}_{i}\right)<\tau=v\left(f_{n}\right)$ then, by Corollary 4 of Theorem $10, \mathfrak{B}_{i}$ is also the $i$ th simple valuation ideal for a rational rank 2 valuation $v^{\prime}$, of type $n$ with respect to $K[x, y]$ and with $f_{1}, \cdots, f_{n}$ as associated polynomials, such that $v^{\prime}\left(\mathfrak{B}_{i}\right)=\tau^{\prime}=v^{\prime}\left(f_{n}\right)$. In short, we may assume without loss of generality that $v\left(\mathfrak{B}_{i}\right) \geqq \tau$.

Let $q$ be the immediate successor of $\mathfrak{B}_{i}$ in the sequence $q_{1}, q_{2}, \ldots$ By Theorem 12, either $v\left(\mathfrak{P}_{i}\right)=s \tau, v(\mathfrak{q})=r / t$ or $v\left(\mathfrak{B}_{i}\right)=r / t, v(\mathfrak{q})=s \tau, s, r$ positive integers, $t=t_{n-1}=\operatorname{deg}_{y} f_{n}$. The two cases are not really different, since, supposing $v\left(\mathfrak{P}_{i}\right)=r / t$, there exists a second valuation $v^{\prime}$ with $f_{1}, \cdots, f_{n}$ as associate polynomials such that $\mathfrak{q}_{1}, \mathfrak{q}_{2}, \cdots, \mathfrak{B}_{i}$ are the first $v^{\prime}$-ideals in $K[x, y]$ and such that $v^{\prime}\left(\mathfrak{B}_{i}\right)=s \tau^{\prime}$, where $\tau^{\prime}=v^{\prime}\left(f_{n}\right)$. We suppose therefore that $v\left(\mathfrak{B}_{i}\right)=s \tau, v(\mathfrak{q})=r / t$. If $F\left(\alpha_{0}, \cdots, \alpha_{n}\right) \in \mathfrak{B}_{i}$ then, just as in the lemma to Theorem 14 , since $v\left(F\left(\alpha_{0}, \cdots, \alpha_{n}\right)\right) \geqq v\left(f_{k}^{s}\right)$, we have

$x^{\alpha(0)} f_{1}^{\alpha(1)}(x, y) \cdots f_{n}^{\alpha(n)}(x, y)=x^{\prime \alpha(0)} f_{1}^{\alpha(1)}\left(x^{\prime}, x^{\prime} y^{\prime}\right) \cdots f_{n}^{\alpha(n)}\left(x^{\prime}, x^{\prime} y^{\prime}\right) \equiv 0\left(x^{\prime s t}\right)$. Also $f_{n}^{s}(x, y)=f_{n}^{s}\left(x^{\prime}, x^{\prime} y^{\prime}\right) \not \equiv 0\left(x^{\prime s t+1}\right)$, whence, from the definition of $T, v\left(T\left(\mathfrak{B}_{i}\right)\right)$ $=v\left(\mathfrak{B}_{i}\right)-s t$. Now $v\left(f_{n}\right)-v\left(f_{n}^{\prime}\right)=t$, whence, placing $v\left(f_{n}^{\prime}\right)=\tau^{\prime}, v\left(T\left(\mathfrak{B}_{i}\right)\right)=s \tau^{\prime}$. By the corollary to Theorem $13, r / s$ is an approximant or quasi approximant to $t \tau$. Since $\tau^{\prime}=\tau-t$, clearly $\left(r-s t^{2}\right) / s$ is an approximant or quasi approximant to $t \tau^{\prime}$. By the corollary to Theorem 13 , then, $s \tau^{\prime}\left(=\min \left(s \tau^{\prime},\left(r-s t^{2}\right) / s\right)\right)$ is the value of a simple $v$-ideal for $v$ in $K[x, y]$. Since $\left(^{8}\right) T\left(\mathfrak{B}_{i}\right)$ is a $v$-ideal, it is therefore a simple $v$-ideal. It remains to prove that $T\left(\mathfrak{B}_{i}\right)$ is the $(i-1)$ th $v$-ideal in $K\left[x^{\prime}, y^{\prime}\right]$.

(8) In the case of a simple $v$-ideal $\mathfrak{B}_{i}$, the proof that $T\left(\mathfrak{B}_{i}\right)$ is a valuation ideal for $v$ is quite immediate. In fact, let $q^{\prime}$ be the $v$-ideal in $K\left[x^{\prime}, y^{\prime}\right]$ having value $s \tau^{\prime}$. We have $T\left(\mathfrak{B}_{i}\right) \subseteq q^{\prime}$ and we wish to prove $q^{\prime} \subseteq T\left(\mathfrak{B}_{i}\right)$. Let $g^{\prime}\left(x^{\prime}, y^{\prime}\right) \in q^{\prime}$. Since $v\left(q^{\prime}\right)=v\left(f_{h}^{\prime \prime}\right)$ and since $f_{n}^{\prime}$ is monic in $y^{\prime}$, reducing $g^{\prime}\left(x, y^{\prime}\right) \bmod f_{n}^{\prime s}$, we may assume $m=\operatorname{deg}_{y^{\prime}} g^{\prime}\left(x^{\prime}, y^{\prime}\right)<\operatorname{deg}_{y^{\prime}} f_{n}^{\prime s}=s t$. There follows: $g^{\prime}\left(x^{\prime}, y^{\prime}\right)$ 
We have shown above that if $v\left(\mathfrak{B}_{i}\right)=v\left(f_{n}^{s}\right)$ then $v\left(T\left(\mathfrak{B}_{i}\right)\right)=v\left(f_{n}^{\prime \prime}\right)$; similarly one shows that if $v\left(\mathfrak{B}_{k}\right)=d_{n-1} v\left(f_{n-1}\right)$ then $v\left(T\left(\mathfrak{P}_{k}\right)\right)=d_{n-1} v\left(f_{n-1}^{\prime}\right)$ whence $v\left(\mathfrak{B}_{k}\right)-v\left(T\left(\mathfrak{B}_{k}\right)\right)=d_{n-1} v\left(f_{n-1}\right)-d_{n-1} v\left(f_{n-1}^{\prime}\right)=d_{n-1} t_{n-2}=t_{n-1}=t$. (The ideal of value $d_{n-1} v\left(f_{n-1}\right)$ is simple by Corollary 2 of Theorem 10.) Also, if $v\left(\mathfrak{B}_{i}\right)=\tau$ then $v\left(\mathfrak{P}_{i}\right)-v\left(T\left(\mathfrak{B}_{i}\right)\right)=t$. Hence if $\mathfrak{q}$ is a $v$-ideal for $v$ with $\mathfrak{B}_{k} \supset \mathfrak{q} \supset \mathfrak{B}_{i}$, the inclusion being proper, then $v(\mathfrak{q})-v(T(\mathfrak{q}))=t$ also, $T\left(\mathfrak{B}_{k}\right) \supset T(\mathfrak{q}) \supset T\left(\mathfrak{B}_{i}\right)$, and each inclusion is proper. Hence there are at least as many $v$-ideals between $T\left(\mathfrak{P}_{k}\right)$ and $T\left(\mathfrak{B}_{i}\right)$ as there are between $\mathfrak{P}_{k}$ and $\mathfrak{P}_{i}$. Since $\mathfrak{q}$ is simple by Corollary 3 of Theorem $10, T(q)$ is simple, and there are at least as many simple $v$-ideals between $T\left(\mathfrak{B}_{k}\right)$ and $T\left(\mathfrak{P}_{i}\right)$ as there are between $\mathfrak{B}_{k}$ and $\mathfrak{B}_{i}$. If, in addition, there are not more $v$-ideals between $T\left(\mathfrak{B}_{k}\right)$ and $T\left(\mathfrak{B}_{i}\right)$ than there are between $\mathfrak{B}_{k}$ and $\mathfrak{B}_{i}$ then the present theorem certainly holds up to $\mathfrak{B}_{i}$, since by induction it holds up to $\mathfrak{B}_{k}$. This is the case. In fact, as we have seen, $v\left(T\left(\mathfrak{P}_{i}\right)\right)=\tau^{\prime}$, and every $v$-ideal in $K\left[x^{\prime}, y^{\prime}\right]$ preceding $T\left(\mathfrak{B}_{i}\right)$ has rational value $u^{\prime} / t, u^{\prime}$ a non-negative integer. For every integer $u^{\prime}$ such that $v\left(T\left(\mathfrak{B}_{k}\right)\right)<u^{\prime} / t<v\left(T\left(\mathfrak{B}_{i}\right)\right)$ there exists, by (a) of Theorem 6 , a $v$-ideal for $v$ in $K\left[x^{\prime}, y^{\prime}\right]$ with value $u^{\prime} / t$. Thus there are as many $v$-ideals between $T\left(\mathfrak{B}_{k}\right)$ and $T\left(\mathfrak{B}_{i}\right)$ as there are integers in the interval $\left(\operatorname{tv}\left(T\left(\mathfrak{B}_{k}\right)\right), \operatorname{tv}\left(T\left(\mathfrak{B}_{i}\right)\right)\right)$. Since $v\left(\mathfrak{B}_{k}\right)-v\left(T\left(\mathfrak{B}_{k}\right)\right)=v\left(\mathfrak{B}_{i}\right)$ $-v\left(T\left(\mathfrak{B}_{i}\right)\right)=t$, an integer, there are the same number of integers in the interval $\left(\operatorname{tv}\left(\mathfrak{B}_{k}\right), \operatorname{tv}\left(\mathfrak{B}_{i}\right)\right)$. By the reasoning just used, this is the number of $v$-ideals between $\mathfrak{B}_{k}$ and $\mathfrak{B}_{i}$, which was to be proved. Hence $T\left(\mathfrak{B}_{i}\right)=\mathfrak{B}_{i_{-1}}^{\prime}$ if $v\left(\mathfrak{B}_{i}\right)=\tau$. Placing $v\left(\mathfrak{B}_{m}\right)=\tau$, we have $T\left(\mathfrak{B}_{m}\right)=\mathfrak{B}_{m-1}^{\prime}$.

Returning to the general case: $v\left(\mathfrak{B}_{i}\right)=s \tau, v(\mathfrak{q})=r / t$, we know that $r / s$ is an approximant or quasi approximant to $t \tau$, say the $k$ th. The correspondence between the simple $v$-ideals and the approximants and quasi approximants to $t \tau$ is such that $\mathfrak{B}_{i}$ is the $(k-2)$ th simple $v$-ideal after $\mathfrak{B}_{m}$ : that is, $i=m+k$ -2 . Since $\left(r-s t^{2}\right) / s$ is the $k$ th approximant or quasi approximant to $t \tau^{\prime}(=t(\tau-t)), T\left(\mathfrak{B}_{i}\right)$ is the $(k-2)$ th simple $v$-ideal after the simple $v$-ideal in $K\left[x^{\prime}, y^{\prime}\right]$ of value $\tau^{\prime}$. Hence $T\left(\mathfrak{B}_{i}\right)=\mathfrak{B}_{m-1+k-2}^{\prime}=\mathfrak{B}_{i-1}^{\prime}$. This completes the proof.

\section{REFERENCES}

1. W. Krull, Allgemeine Bewertungstheorie, Journal für Mathematik vol. 167 (1932).

2. Saunders MacLane and O. F. G. Schilling, Zero-dimensional branches of rank one on algebraic varieties, Ann. of Math. vol. 40 (1939).

3. A. Ostrowski, Untersuchung zur arithmetische Theorie der Körper, part 3, Math. Zeit. vol. 39 (1934).

4. O. Perron, Die Lehre von den Kettenbrüchen, Leipzig and Berlin, 1913.

5. O. Zariski, Polynomial ideals defined by infinitely near base points, Amer. J. Math. vol. 60 (1938).

Williams College,

Williamstown, Mass.

$=g^{\prime}(x, y / x)=g(x, y) / x^{m}, g(x, y) \in \mathcal{D}$, whence $g^{\prime}\left(x^{\prime}, y^{\prime}\right)$ arises under $T$ from $g(x, y) x^{s t-m}$. This last element is in $\mathfrak{B}_{i}$ since it has value $=v\left(g^{\prime}\right)+s t \geqq v\left(f_{n}^{\prime \prime}\right)+s t=v\left(f_{n}^{\prime \prime}\right)$. 